\title{
Evaluierung der Dienstleistungen des AMS Jugendliche für Personen mit Migrationshintergrund
}

Projektleitung AMS Wien:

Gerda Challupner

Claudia Felix

Ali Ordubadi

Projektleitung KMU FORSCHUNG AUSTRIA:

Eva Heckl

KMU FORSCHUNG AUSTRIA

Austrian Institute for SME Research

Wien, Jänner 2011 
KMU FORSCHUNG AUSTRIA

Austrian Institute for SME Research

Diese Studie wurde im Auftrag des Arbeitsmarktservice (AMS) Wien durchgeführt.

Verfasserinnen des Berichts:

Eva Heckl (Projektleitung)

Céline Dörflinger

Andrea Dorr

Internes Review/Begutachtung:

Sonja Sheikh

Layout:

Susanne Fröhlich

Martina Gugerell

Mitglied bei / Member of
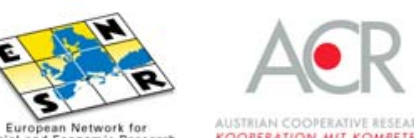

Die vorliegende Studie wurde nach allen Maßstäben der Sorgfalt erstellt.

Die KMU FORSCHUNG AUSTRIA übernimmt jedoch keine Haftung für Schäden oder Folgeschäden, die auf diese Studie oder auf mögliche fehlerhafte Angaben zurückgehen.

Dieses Werk ist urheberrechtlich geschützt. Jede Art von Nachdruck, Vervielfältigung, Verbreitung, Wiedergabe, Übersetzung oder Einspeicherung und Verwendung in Datenverarbeitungssystemen, und sei es auch nur auszugsweise, ist nur mit ausdrücklicher Zustimmung der KMU FORSCHUNG AUSTRIA gestattet. 


\section{Inhaltsverzeichnis}

Zusammenfassung 1

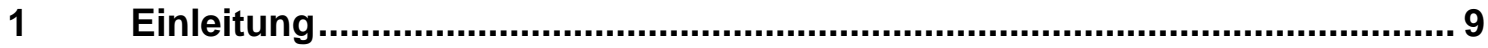

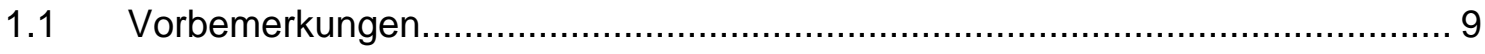

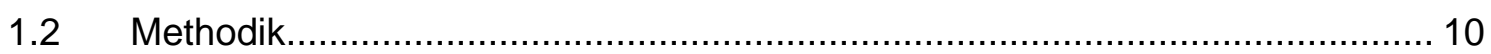

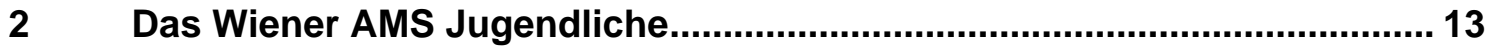

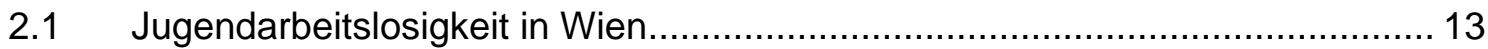

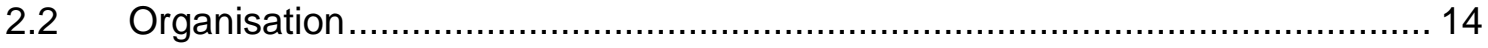

2.3 Diversity Management im Rahmen des Wiener AMS Jugendliche .................. 18

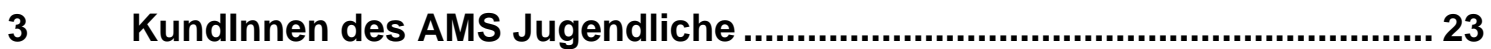

3.1 Charakteristika der Jugendlichen mit Migrationshintergrund ........................... 23

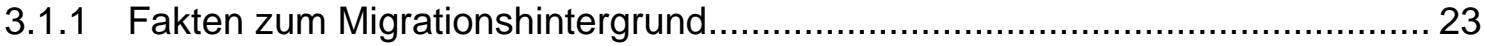

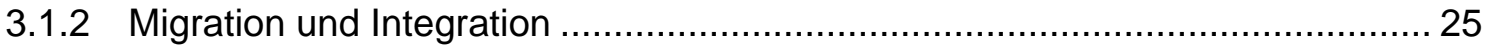

3.2 Beschäftigungssituation und Ausbildungshintergrund der Eltern.................... 29

$4 \quad$ Ausbildungshintergrund und Integration am Arbeitsmarkt......................... 35

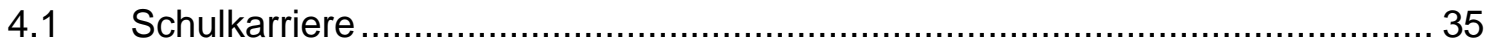

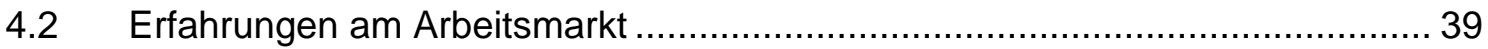

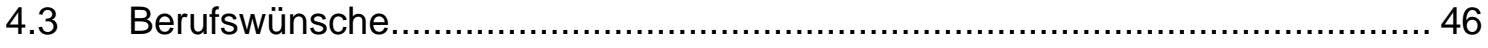

4.4 Die Arbeitsmarktintegration beeinflussende Faktoren .................................. 48

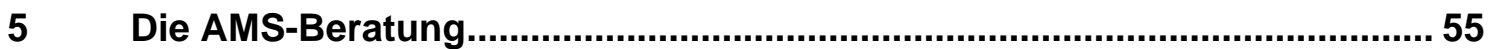

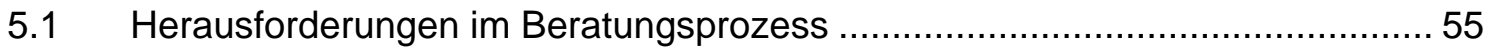

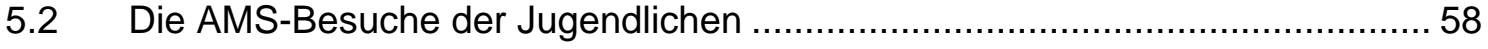

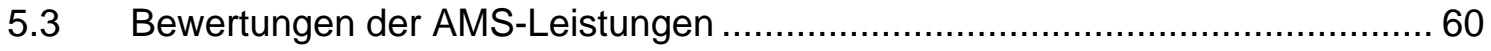

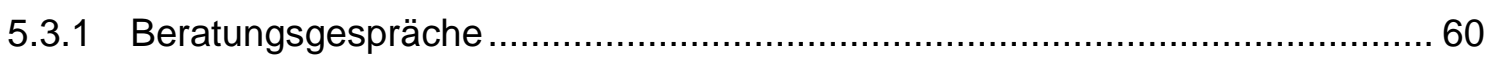

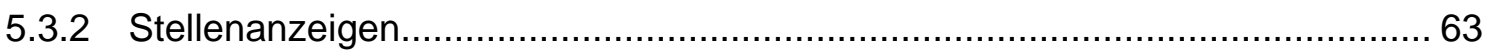

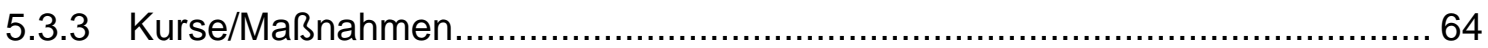

5.3.4 Allgemeine Zufriedenheit und Wünsche an das AMS ................................. 72

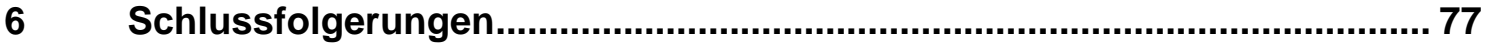




\section{Grafikverzeichnis}

Grafik 1 Beurteilung des aktuellen Standorts im Vergleich zum alten Standort, Anteil der befragten Jugendlichen, die beide Standorte kennen, in Prozent.

Grafik 2 Attraktivität des Informationsmaterials, Anteil der befragten Jugendlichen, die das Material nutzen, in Prozent

Grafik 3 Wahrgenommene Verbesserung des Informationsmaterials, Anteil der befragten Jugendlichen, die das Material nutzen, in Prozent

Grafik 4 Subjektive Wahrnehmung der Behandlung der Jugendlichen mit Migrationshintergrund seitens der AMS-BeraterInnen, Anteil der befragten Jugendlichen nach Migrationshintergrund in Prozent.

Grafik 5 Art des Migrationshintergrundes der Jugendlichen, Anteil der migrantischen Jugendlichen in Prozent.....

Grafik 6 Geburtsort der Jugendlichen, Anteil der migrantischen Jugendlichen nach Migrationshintergrund in Prozent.

Grafik 7 Alter zum Zeitpunkt der Immigration der im Ausland geborenen Jugendlichen, Anteil der migrantischen Jugendlichen nach Altersgruppen und Migrationshintergrund in Prozent

Grafik 8 Staatsbürgerschaft der Jugendlichen, Anteil der migrantischen Jugendlichen nach Migrationshintergrund in Prozent.

Grafik 9 Gesprochene Sprache mit den Eltern, Anteil der migrantischen Jugendlichen nach Generation und Migrationshintergrund in Prozent .... 26

Grafik 10 Gesprochene Sprache mit den Geschwistern, Anteil der migrantischen Jugendlichen nach Generation und Migrationshintergrund in Prozent .... 26

Grafik 11 Gesprochene Sprache mit den Freunden, Anteil der migrantischen Jugendlichen nach Generation und Migrationshintergrund in Prozent .... 27

Grafik 12 Pflege der Traditionen des Herkunftslandes in Bezug auf die Feiertage, Anteil der migrantischen Jugendlichen nach Migrationshintergrund in Prozent....

Grafik 13 Zugehörigkeitsgefühl der Jugendlichen, Anteil der migrantischen Jugendlichen nach Generation in Prozent......

Grafik 14 Erwerbsstatus der Väter der Jugendlichen, Anteil der befragten Jugendlichen nach Migrationshintergrund in Prozent.

Grafik 15 Höchst abgeschlossene Ausbildung der Väter der Jugendlichen, Anteil der befragten Jugendlichen nach Migrationshintergrund in Prozent.

Grafik 16 Erwerbsstatus der Mütter der Jugendlichen, Anteil der befragten Jugendlichen nach Migrationshintergrund in Prozent.....

Grafik 17 Höchst abgeschlossene Ausbildung der Mütter der Jugendlichen, Anteil der befragten Jugendlichen nach Migrationshintergrund in Prozent.

Grafik 18 Zuletzt besuchte Schule der Jugendlichen, Anteil der befragten Jugendlichen nach Migrationshintergrund in Prozent.... 
Grafik 19 Unterstützung der Jugendlichen beim Lernen während der Schulzeit, Anteil der befragten Jugendlichen nach Migrationshintergrund in Prozent......

Grafik 20 Möglichkeit eines ungestörten Lernens der Jugendlichen zuhause, Anteil der befragten Jugendlichen nach Migrationshintergrund und Geschlecht in Prozent.

Grafik 21 Häufigkeit der Kontakte der Eltern mit der Schule, Anteil der befragten Jugendlichen nach Migrationshintergrund in Prozent.

Grafik 22 Überblick der Eltern über das Schul- und Ausbildungssystem in Österreich, Anteil der befragten Jugendlichen nach Migrationshintergrund in Prozent

Grafik 23 Berufliche Erfahrungen der Jugendlichen, Anteil der befragten Jugendlichen nach Migrationshintergrund und Geschlecht in Prozent

Grafik 24 Art der beruflichen Erfahrungen der Jugendlichen, Anteil der befragten Jugendlichen nach Migrationshintergrund und Geschlecht in Prozent

Grafik 25 Darstellung des Lehrausbildungsverlaufs der Jugendlichen, Anteil der befragten Jugendlichen in Prozent

Grafik 26 Darstellung des Lehrausbildungsverlaufs der Jugendlichen, Anteil der befragten Jugendlichen nach Geschlecht in Prozent

Grafik 27 Darstellung des Lehrausbildungsverlaufs der Jugendlichen, Anteil der befragten Jugendlichen nach Migrationshintergrund in Prozent.

Grafik 28 Top 7 der Berufsbereiche der männlichen Jugendlichen, Anteil der befragten männlichen Jugendlichen nach Migrationshintergrund in Prozent

Grafik 29 Top-7 der Berufsbereiche der weiblichen Jugendlichen, Anteil der befragten weiblichen Jugendlichen nach Migrationshintergrund in Prozent

Grafik 30 Wunschberufsbereiche der Jugendlichen, Anteil der befragten Jugendlichen nach Geschlecht in Prozent.

Grafik 31 Hilfe bei der Berufsfindung der Jugendlichen, Anteil der befragten Jugendlichen nach Migrationshintergrund in Prozent....

Grafik 32 Informationsquellen der Jugendlichen für die Berufswahl, Anteil der befragten Jugendlichen nach Migrationshintergrund in Prozent.

Grafik 33 Informationsquellen der Jugendlichen für die Arbeitssuche, Anteil der befragten Jugendlichen nach Migrationshintergrund in Prozent.

Grafik 34 Erforderliche Fähigkeiten, Kenntnisse und Rahmenbedingungen, um eine Arbeit zu finden, Anteil der befragten Jugendlichen nach Migrationshintergrund in Prozent.

Grafik 35 Persönliche Probleme am Arbeitsmarkt, Anteil der befragten Jugendlichen nach Migrationshintergrund in Prozent.

Grafik 36 Gefühl der Jugendlichen, dass sie bei der Arbeits-/Lehrstellensuche mehr Schwierigkeiten haben als andere, Anteil der befragten Jugendlichen nach Migrationshintergrund in Prozent..... 
Grafik 37 Vormerkdauer beim AMS nach Migrationshintergrund, Anteil der befragten Jugendlichen nach Migrationshintergrund in Prozent

Grafik 38 Impulsgeber für den ersten AMS-Besuch nach Migrationshintergrund, Anteil der befragten Jugendlichen nach Migrationshintergrund in Prozent

Grafik 39 Gründe für den AMS-Besuch, Anteil der befragten Jugendlichen nach Migrationshintergrund in Prozent

Grafik 40 Bewertung des letzten Beratungsgesprächs, Anteil der befragten Jugendlichen, die mindestens ein Beratungsgespräch hatten, nach Migrationshintergrund in Prozent

Grafik 41 Zufriedenheit mit den AMS-BeraterInnen, Anteil der befragten Jugendlichen, die mindestens ein Beratungsgespräch hatten, nach Migrationshintergrund in Prozent

Grafik 42 Übereinstimmung der Stellenanzeigen des AMS mit den Berufswünschen der Jugendlichen, Anteil der befragten Jugendlichen, die Stellen erhalten haben, nach Migrationshintergrund in Prozent

Grafik 43 Art der besuchten Kurse/Maßnahmen, Anteil der befragten Jugendlichen, die bereits an einem Kurs teilgenommen haben, in Prozent.

Grafik 44 Art des Zustandekommens der letzten zwei Kursbesuche, Anteil der Kursbesuche in Prozent ${ }^{1}$

Grafik 45 Ausreichend Informationen vor dem Kursbesuch erhalten, Anteil der Kursbesuche in Prozent ${ }^{1}$

Grafik 46 Erfolgreicher Kursabschluss, Anteil der Kursbesuche in Prozent.

Grafik 47 Bewertung der letzten zwei Kurse nach deren Nützlichkeit, Anteil der Kursbesuche in Prozent ${ }^{1}$

Grafik 48 Zufriedenheit mit dem AMS insgesamt, Anteil der befragten Jugendlichen nach Migrationshintergrund in Prozent....

Grafik 49 Wünsche an das AMS, Anteil der befragten Jugendlichen nach Migrationshintergrund in Prozent 


\section{Tabellenverzeichnis}

Tabelle 1 Allgemeine Charakteristika der Stichprobe

Tabelle 2 Vorgemerkte arbeitslose Jugendliche am AMS Jugendliche, in Wien und Österreich, 2005 bis 2009

Tabelle 3 Lehrstellenmarkt in Wien und Österreich, 2005 bis 2009 14

Tabelle 4 Berufsbereiche der Väter der Jugendlichen, Anteil der befragten Jugendlichen nach Migrationshintergrund in Prozent.

Tabelle 5 Berufsbereiche der Mütter der Jugendlichen, Anteil der befragten Jugendlichen nach Migrationshintergrund in Prozent

Tabelle 6 Top-10 Lehrberufe der Jugendlichen, Anteil der befragten Jugendlichen nach Geschlecht in Prozent. 


\section{Zusammenfassung}

Personen mit Migrationshintergrund stellen eine bedeutende Klientlnnengruppe des Wiener Arbeitsmarktservice (AMS) für Jugendliche dar. Das AMS Jugendliche versucht, die Leistungen und Beratungen auf die speziellen Bedürfnisse dieser Zielgruppe anzupassen sowie allgemein laufend zu optimieren. Daher wurde die KMU FORSCHUNG AUSTRIA wie schon im Jahr 2007 neuerlich beauftragt, das Beratungsangebot und den Beratungsprozess im AMS Jugendliche zu analysieren, die Charakteristika und arbeitsmarktrelevanten Parameter der Zielgruppe zu untersuchen sowie die Wirkungen der organisatorischen Neuerungen zu überprüfen. Dabei erfolgte eine Analyse der Unterschiede zwischen Jugendlichen mit und ohne Migrationshintergrund sowie der Veränderungen im Vergleich zur Ersterhebung im Jahr 2007. Operativ wurde ein Mix aus qualitativen (teilnehmende Beobachtung, Workshops mit AMS-MitarbeiterInnen, Fokusgruppen mit jugendlichen AMS-KundInnen) und quantitativen Methoden (Primärerhebung) gewählt.

\section{Das Wiener AMS Jugendliche}

Am Wiener AMS Jugendliche wurden im Jahr 2009 rd. 12.600 jugendliche KundInnen unter 21 Jahren betreut. In den vergangenen Jahren war - mit Ausnahme von 2006 ein kontinuierlicher Anstieg an vorgemerkten arbeitslosen Jugendlichen zu beobachten, da auch die Situation am Lehrstellenmarkt angespannt bleibt. Die Lehrstellenandrangsziffer ist in Wien mit 4,2 im Jahr 2009 weitaus höher als im Österreich-Durchschnitt. Auf Grund der steigenden Anzahl an zu betreuenden Jugendlichen, die eine Arbeit oder eine Lehrstelle suchen, wurde Ende 2009 ein Umzug in ein neues, deutlich größeres Haus am Gumpendorfer Gürtel erforderlich. Die Übersiedlung war von internen Umstrukturierungsprozessen, wie z. B. der neuen Aufteilung der Kundengruppen zwischen den AMS-Zonen und einem neuen Terminvereinbarungssystem, begleitet. Die neuen Räumlichkeiten haben die Beratungsbedingungen und -atmosphäre sowohl für die AMS-BeraterInnen als auch für die Jugendlichen erheblich verbessert. Durch die Neuorganisation der Terminvergabe konnten die Wartezeiten deutlich reduziert werden, jedoch war es dadurch nicht möglich, die Spontanvorsprachen der jungen KundInnen, die ihre Termine vergessen haben oder nicht einhalten, einzudämmen.

Am Wiener AMS Jugendliche wird auf Diversity Management großer Wert gelegt und es fließt als Querschnittsmaterie in alle Kernprozesse mit ein. Ein wesentliches Handlungsfeld im Rahmen des Diversity Managements bezieht sich auf Informationsaktivitäten für Jugendliche und deren Eltern. Mehr als die Hälfte der befragten Jugendlichen (56 \%) nimmt Informationsmaterialien des AMS in Anspruch, wobei sie in erster Linie auf die AMS-Homepage zugreifen und weniger Broschüren oder schriftliche Materialien heranziehen. Dem Großteil der KundInnen, welche die Informationsangebote nutzen, gefallen diese, lediglich ein knappes Zehntel (insbesondere Jugendliche mit Migrationshintergrund) hat Schwierigkeiten, die Inhalte zu verstehen. Um geeignete Rahmenbedingungen in der Beratungssituation zu schaffen, werden verstärkt Mitarbeiterlnnen mit Migrationshintergrund aufgenommen. Trotzdem wird nur sehr selten das gesamte Beratungsgespräch in einer anderen Sprache abgewickelt, auch wenn dies grundsätzlich mehr als ein Drittel der befragten Jugendlichen (36\%) sinnvoll fände. Darüber hinaus nehmen einige AMS-BeraterInnen Weiterbildungsmaßnahmen zum Thema Diversity-Management in Anspruch. Auch für die Zielgruppe der Jugendlichen mit Migrationshintergrund stehen zahlreiche spezifische Qualifizierungsmaßnahmen zur Verfügung. 


\section{Charakteristika der Jugendlichen mit Migrationshintergrund am AMS}

Im Mittelpunkt der Studie steht die Befragung von Jugendlichen, die im AMS Jugendliche Beratung bzw. Serviceleistungen in Anspruch nehmen. Personen mit Migrationshintergrund wurden für den Zweck der Studie definiert als Personen, die

- eine ausländische Staatsbürgerschaft besitzen und/oder

- im Ausland geboren sind und/oder

- deren Eltern im Ausland geboren sind und/oder

- deren Umgangssprache mit der Familie eine andere Sprache als Deutsch ist.

Insgesamt wurden 431 Personen interviewt, davon 311 Jugendliche mit Migrationshintergrund und 120 Jugendliche ohne Migrationshintergrund. Der Anteil an weiblichen Jugendlichen unter allen Befragten lag bei $44 \%$. Ein Drittel der Jugendlichen war zum Befragungszeitpunkt unter 17 Jahre alt und $20 \%$ war 18 Jahre alt. Der Anteil der über 19-Jährigen lag bei $47 \%$. In der Stichprobe befanden sich vor allem Personen aus dem ehemaligen Jugoslawien und der Türkei (jeweils rd. ein Drittel), sonstige befragte Personen stammen aus Osteuropa und Russland, dem Nahen und Mittleren Osten, Afrika oder Asien. Rd. zwei Drittel (64 \%) der befragten jugendlichen MigrantInnen sind in Österreich und knapp ein Drittel im Ausland geboren. Insgesamt besitzen rd. zwei Drittel der befragten MigrantInnen die österreichische Staatsbürgerschaft. Der Großteil der Jugendlichen (48\%), die im Ausland geboren sind, kam im Alter von 7 bis 15 Jahren nach Österreich. Rd. $32 \%$ kamen vor dem 6. Lebensjahr und ein Fünftel nach dem 15. Lebensjahr nach Österreich.

Die Beherrschung und der Gebrauch der deutschen Sprache sind wichtige Vorraussetzungen für die Integration in den österreichischen Arbeitsmarkt und stellen somit wichtige Integrationsfaktoren dar. $44 \%$ der befragten MigrantInnen gaben an, dass sie mit ihren Eltern ausschließlich in ihrer Muttersprache kommunizieren. Bei den Jugendlichen der ersten Generation liegt dieser Anteil bei $64 \%$. Bei $47 \%$ der Befragten wird im Familienkreis mehr als eine Sprache gesprochen. Deutsch ist hier in den häufigsten Fällen die Zweitsprache. Mit den Geschwistern spricht mehr als ein Drittel (36\%) der Jugendlichen nur Deutsch, insbesondere die Jugendlichen der zweiten Generation (47\%). Bei mehr als der Hälfte der befragten MigrantInnen (57\%) werden im Freundeskreis mehrere Sprachen gesprochen, insbesondere bei den Jugendlichen aus ExJugoslawien (63 \%) und der Türkei (67\%). Mehr als ein Drittel der MigrantInnen spricht ausschließlich Deutsch mit seinen FreundInnen.

Die jugendlichen MigrantInnen wurden ebenfalls zur Rolle der Traditionen des Herkunftslandes innerhalb ihrer Familien befragt und insbesondere über die Pflege der Feiertage, der Essgewohnheiten oder von etwaigen Bekleidungsvorschriften. Insgesamt gaben $89 \%$ der befragten MigrantInnen an, dass in ihre Familie die Traditionen ihres Herkunftslandes pflegen, in erster Linie nannten diese Jugendlichen die Einhaltung der Feiertage (90\%). Weitere $89 \%$ gaben an, dass die Essgewohnheiten des Herkunftslandes in der Familie einen festen Platz haben, insbesondere in Familien aus der Türkei, dem Nahen und Mittleren Osten sowie aus Afrika. Bekleidungsvorschriften spielen in rd. einem Drittel der Familien eine Rolle (36\%), am häufigsten in Familien aus der Türkei (51\%) und dem Nahen bzw. Mittleren Osten (45\%), d. h. aus vom Islam geprägten Kulturkreisen. 


\section{Beschäftigungs- und Ausbildungssituation der Eltern}

Hinsichtlich der Arbeitsmarkt- und Ausbildungssituation gaben fast $80 \%$ der befragten Jugendlichen an, dass ihre Väter in aufrechten Arbeitsverhältnissen stehen. Bei den Vätern der Jugendlichen ohne Migrationshintergrund waren es $85 \%$. Der Großteil der Väter - und insbesondere jene mit Migrationshintergrund - arbeitet im Bauwesen und Baunebengewerbe, gefolgt vom Verkehr/Transport/Zustelldienste. In Bezug auf die Erwerbstätigkeit der Mütter gibt es deutliche Unterschiede nach Herkunftsgruppe. Insgesamt ist mehr als die Hälfte der Mütter erwerbstätig (55\%), insbesondere aber die österreichstämmigen Mütter (81\%) und jene aus Ex-Jugoslawien (69 \%). Lediglich ein Drittel der türkischen Mütter ist am Arbeitsmarkt integriert. Während die migrantischen Mütter am häufigsten in der Reinigung/Haubetreuung, im Hotel- und Gastgewerbe und der Gesundheit/Medizin arbeiten, sind die österreichstämmigen Mütter in erster Linie im Büro/Wirtschaft und im Handel/Verkauf tätig.

Das Ausbildungsniveau der migrantischen Eltern und insbesondere der Mütter ist insgesamt deutlich niedriger als das der österreichstämmigen Eltern. Während bei den österreichischen Eltern die Mehrheit über einen Lehrabschluss verfügt, dominiert bei den migrantischen Eltern der Pflichtschulabschluss.

\section{Ausbildungshintergrund und Arbeitsmarktsintegration}

Eine Grundausbildung sowie entsprechende Schulabschlüsse sind wichtige Faktoren, um die Integration von Jugendlichen am Arbeitsmarkt zu erleichtern. Ein wesentliches Problem der Jugendlichen, die beim AMS gemeldet sind, ist ihr niedriges Ausbildungsniveau. Hier zeigt sich, dass die niedrige Ausbildung der Eltern an die Kinder „weitervererbt" wird. Die Befragung ergab, dass rd. $43 \%$ der befragten Jugendlichen zuletzt die Hauptschule bzw. eine kooperative Mittelschule besucht haben. Im Polytechnischen Lehrgang waren knapp ein Viertel (23\%) der Befragten. Jeweils ein Zehntel der Jugendlichen hat eine berufsbildende mittlere oder höhere Schule besucht und lediglich $9 \%$ die AHS. Insgesamt haben $67 \%$ der befragten Jugendlichen - unabhängig von Migrationshintergrund - die zuletzt besuchte Schule positiv abgeschlossen.

Die Ergebnisse zeigen, dass die befragten Jugendlichen bereits während der Schulzeit mehr Unterstützung benötigt hätten, um einen besseren Schulabschluss zu erreichen und ihre Chancen am Arbeitsmarkt zu steigern. Rd. ein Drittel der befragten Jugendlichen (36\%) wurde während ihrer Schulzeit beim Lernen unterstützt. Am häufigsten hatten sie Hilfe von Familienmitgliedern, insbesondere österreichstämmige Jugendliche (64\% vs. $43 \%$ der migrantischen Jugendlichen). Mehr als die Hälfte der befragten Jugendlichen (51\%) gab an, dass sie mehr Unterstützung gebraucht hätte. Die mangelnde Unterstützung und auch der fehlende regelmäßige Kontakt zwischen den Eltern der Jugendlichen und der Schule, insbesondere bei migrantischen Eltern, erschwerten außerdem einen positiven Schulabschluss.

Trotz ihres jungen Alters haben insgesamt $79 \%$ der befragten Jugendlichen bereits gearbeitet. Die österreichstämmigen (86 \%) und die männlichen (83 \%) Jugendlichen häufiger als die migrantischen (76\%) und die weiblichen (74\%) Jugendlichen. Mehr als die Hälfte (57\%) der befragten Jugendlichen gab an, bereits einmal eine Lehrausbildung begonnen zu haben, $47 \%$ davon in einem Betrieb und $53 \%$ in einer Ausbildungseinrichtung. Rd. ein Viertel hat die Lehre abgeschlossen und rd. ein Zehntel befindet sich noch in der Lehrausbildung. Fast die Hälfte dieser Jugendlichen (48\%) will in diesem Beruf auch in Zukunft tätig sein. Weitere $46 \%$ wollen einen Berufswechsel vornehmen und in einem anderen Bereich in Zukunft arbeiten. Insgesamt 
brachen aber fast zwei Drittel (63\%) die Lehrausbildung ab. Die Abbruchsquote ist in den Ausbildungseinrichtungen (74\%) weit höher als in den Betrieben (53\%). $64 \%$ der LehrabbrecherInnen wollen zudem in Zukunft einen anderen Beruf wählen. Ein Drittel jedoch will in demselben Lehrberuf weiter tätig sein bzw. eine neue Lehrstelle suchen.

Während $64 \%$ der männlichen Jugendlichen eine Lehre begonnen haben, sind es bei den Mädchen nur $47 \%$. Daneben ist der Anteil der Mädchen, die die Lehre in einer Ausbildungseinrichtung begonnen haben, höher (58\% vs. $50 \%$ ). Die Lehre positiv abgeschlossen haben die Mädchen aber im selben Ausmaß wie die Buben (rd. ein Viertel). Zwischen den Jugendlichen mit und ohne Migrationshintergrund bestehen ebenfalls Unterschiede im Bezug auf die Erfahrungen mit der Lehrausbildung. Während $68 \%$ der österreichstämmigen Jugendlichen eine Lehre bereits einmal begonnen haben, sind es unter den migrantischen Jugendlichen nur $53 \%$. Letztere haben, wenn sie eine Lehre absolviert haben, diese auch öfters in Ausbildungseinrichtungen gemacht als die österreichstämmigen Jugendlichen (55 \% vs. $49 \%$ ).

Die Wahl der Lehrberufe bzw. der Berufsbereiche ist je nach Geschlecht und Migrationshintergrund unterschiedlich. Männliche Migranten sind vor allem im Bau/Baunebengewerbe und im Berufsbereich Maschinen/Kfz/Metall sowie Hotel- und Gastgewerbe tätig. Die österreichstämmigen männlichen Jugendlichen sind neben diesen genannten Berufsbereichen aber auch vermehrt im Handel/Verkauf, im Büro/Wirtschaft und Informationstechnologie vorzufinden. Während Migrantinnen in erster Linie im Handel/Verkauf und im Hotel- und Gastgewerbe vorzufinden sind, wählen die österreichstämmigen Mädchen die Körper- und Schönheitspflege noch vor dem Handel/ Verkauf. Wie auch bei den Buben sind letztere auch häufiger im Berufsbereich Büro/ Wirtschaft tätig als die Mädchen mit Migrationshintergrund.

In Hinblick auf die Berufswünsche der Jugendlichen, die noch keinen Beruf gewählt haben, besteht wie bei den anderen Jugendlichen eine starke geschlechtsspezifische Segmentierung. Während die Buben zu technischen und handwerklichen Berufe tendieren (wie z. B. Berufsbereiche wie Maschinen/Kfz/Metall oder Bau), bevorzugen Mädchen den Handel/Verkauf oder Ausbildungen im Bürobereich oder im Bereich der Gesundheit/Medizin.

Mehr als die Hälfte der befragten Jugendlichen gab an, dass sie sich bezüglich ihrer Berufswahl alleine entschieden haben. Rd. ein Fünftel hat sich aber mit den Eltern beraten, insbesondere die österreichstämmigen Jugendlichen. Rd. ein Zehntel wurde von FreundInnen beeinflusst. Rd. $8 \%$ der migrantischen Jugendlichen haben außerdem von BeraterInnen Hilfe in Anspruch genommen. Als konkrete Informationsquellen für die Berufswahl nannten rd. ein Drittel der Jugendlichen das AMS, insbesondere die MigrantInnen (37\% vs. $27 \%$ der österreichstämmigen Jugendlichen). Die österreichstämmigen Jugendlichen informierten sich hingegen häufiger im Internet (35\% vs. $24 \%$ der MigrantInnen). Berufsorientierungskurse wurden in erster Linie von den MigrantInnen als Informationsquelle genutzt. Rd. ein Drittel der befragten Jugendlichen gab an, das sie das Berufsinformationszentrum (BIZ) im AMS (einmal) besucht haben. Rd. die Hälfte jener Jugendlichen (49\%), die das BIZ besucht haben, hat dies mit der Schule gemacht. Für jene Jugendlichen, die das BIZ besucht haben, war es für mehr als zwei Drittel (69\%) hilfreich und insbesondere für die migrantischen Jugendlichen (73 \% vs. $59 \%$ der österreichstämmigen Jugendlichen).

Um am Arbeitsmarkt Fuß zu fassen, sucht der Großteil der Jugendlichen (91\%) selbstständig nach freien Stellen, wobei vor allem das Internet herangezogen wird. Während österreichische Jugendliche neben dem Internet häufiger in Zeitungen und 
Zeitschriften nach Arbeitsmöglichkeiten als MigrantInnen suchen, wenden sich letztgenannte öfter an den Freundes- und Bekanntenkreis und schätzen vor allem die persönliche Vorsprache direkt bei den Unternehmen.

Als entscheidender Faktor, um bei der Arbeitssuche erfolgreich zu sein, wird eine gute Ausbildung genannt, wobei von jeweils rd. einem Fünftel der befragten Jugendlichen vor allem gute Deutschkenntnisse und ein positiver Hauptschulabschluss betont werden. Des Weiteren sind für die Jugendlichen der eigene (Arbeits-)Wille, die Motivation und die Eigeninitiative bei der Stellensuche sowie verschiedene Soft Skills und Arbeitstugenden für eine erfolgreiche Arbeitsmarktintegration ausschlaggebend. Mit Schwierigkeiten am Arbeitsmarkt haben 2010 mehr Jugendliche zu kämpfen als im Jahr 2007 (81 \% vs. $65 \%$ ), wobei sie sich vor allem einem Mangel an freien Lehrstellen und Arbeitsplätzen bzw. einer großen Konkurrenz an Mitbewerberlnnen gegenüber sehen. Auch fehlende Schul- und Ausbildungsabschlüsse (insbesondere bei MigrantInnen) sowie Motivationsdefizite erschweren den Vermittlungsprozess. Insgesamt hat etwa ein Drittel der befragten Jugendlichen den Eindruck, dass sie es beim Bewerbungsprozess schwieriger haben als andere junge Menschen.

\section{Der Beratungsprozess}

Die Beratung jugendlicher AMS-KundInnen mit Migrationshintergrund zählt zum Berufsalltag der AMS-BeraterInnen. Besondere Herausforderungen im Beratungsprozess stellen bei dieser Zielgruppe sprachliche Barrieren, der Umgang mit kulturellen Traditionen (z. B. Kopftuch), Mentalitätsunterschiede sowie fehlende bzw. nicht anerkannte Pflichtschulabschlüsse dar. Auch wenn die Eltern zu viel oder zu wenig Einfluss nehmen, die Jugendlichen keine Vorstellung über mögliche berufliche Perspektiven haben und ihre Situation am Arbeitsmarkt nicht realistisch einschätzen, ist oft umfassende Aufklärungsarbeit im Rahmen der Beratungsgespräche erforderlich.

Beinahe die Hälfte der Jugendlichen (47\%) war zum Zeitpunkt der Befragung länger als 6 Monate arbeitslos, etwa ein Viertel sogar länger als ein Jahr, wobei MigrantInnen tendenziell etwas länger arbeitslos gemeldet sind als Jugendliche ohne Migrationshintergrund. Die Jugendlichen wenden sich zumeist aus Eigeninitiative (35\%), auf Anraten der Eltern/der Familie (34\%) oder der Schule (19\%) an das AMS. Während sich österreichische Jugendliche vor allem aus eigenem Antrieb oder auf Empfehlung ihrer Eltern das AMS aufsuchen, sind MigrantInnen stärker auf Informationen von der Schule sowie von Freunden und Bekannten angewiesen. Hauptgrund für den Besuch des AMS stellt die Suche nach einer Arbeit (53\%) oder einer Lehrstelle (33 \%) dar.

Die befragten Jugendlichen sind sowohl mit den Beratungsgesprächen als auch mit ihren BeraterInnen (sehr) zufrieden. Mehr als $60 \%$ der befragten Jugendlichen hat ihr letztes Gespräch sehr oder etwas geholfen. Diese waren für die KundInnen vor allem dann eine Hilfe, wenn Kursbesuche vereinbart, Stellenanzeigen für freie Arbeitsplätze oder Lehrstellen sowie Informationen für den Bewerbungsprozess mit- bzw. weitergegeben wurden. Auch die Zufriedenheit mit dem/r jeweiligen Beraterln ist sehr hoch, wie $83 \%$ der befragten AMS-KundInnen angeben. Diese werden als nett und freundlich beschrieben und die Jugendlichen schätzen, dass sie auf ihre Berufswünsche und Probleme eingehen, sie motivieren und sich um sie kümmern.

Hingegen ist für beinahe $40 \%$ der AMS-KundInnen das letzte Beratungsgespräch weniger oder überhaupt nicht hilfreich verlaufen, da keine geeigneten Stellen gefunden wurden, der gewünschte Kursbesuch nicht zustande kam und keine Neuigkeiten zu erfahren waren, sondern vorwiegend administrative Angelegenheiten und Formalitäten 
abgewickelt wurden. Mit dem/r Beraterln sind lediglich $17 \%$ der Jugendlichen weniger oder nicht zufrieden. Diese negative Beurteilung kommt dann zustande, wenn die AMS-MitarbeiterInnen als unfreundlich, distanziert oder streng erlebt werden, die Jugendlichen sich nicht verstanden, alleine gelassen oder unter Druck gesetzt fühlen, häufige BeraterInnenwechsel stattfinden und die Gespräche in zu kurzer Zeit abgewickelt werden.

Eine weitere Aufgabe der AMS-BeraterInnen ist es, den KundInnen regelmäßig Stellenanzeigen mitzugeben. Bei einem Drittel stimmten die Stellen mit den Berufswünschen überein. $38 \%$ bzw. $28 \%$ bekamen Inserate, die nur teilweise oder überhaupt nicht ihren beruflichen Vorstellungen entsprachen. Die mangelnde Übereinstimmung betraf in erster Linie die gewählte Branche oder die angestrebten Berufe, aber auch den Umfang des Arbeitsverhältnisses (Teilzeit/Vollzeit) sowie die mitzubringenden Voraussetzungen und die Anforderungen bei der ausgeschriebenen Stelle.

Auf Grund der niedrigen Qualifikation oder weil die Jugendlichen nicht unmittelbar ,jobready" sind, werden sie oftmals in Weiterbildungsaktivitäten vermittelt, wie dies auch bereits bei $59 \%$ der befragten Jugendlichen der Fall war. Jugendliche mit Migrationshintergrund nahmen etwas häufiger an Qualifizierungsaktivitäten des AMS teil als österreichische Jugendliche (62\% vs. $51 \%$ ). Am häufigsten wurden Maßnahmen zur Berufsorientierung und Vorbereitung auf den Arbeitsmarkt (53\%) besucht, gefolgt von Lehr- und Berufsausbildungen (24\%), Büro-/EDV-Kursen (14\%), Deutschkursen (12\%) sowie Maßnahmen zum Erwerb von Zusatzqualifikationen (13\%). Diese Kursbesuche kamen in etwas mehr als der Hälfte der Fälle auf Vorschlag des/r AMS-Beraters/in zustande, wobei die Beraterlnnen bei rd. zwei Drittel der Maßnahmenteilnahmen die KundInnen vorab ausreichend über Inhalt und Zweck informierten. Die Jugendlichen haben insgesamt rd. zwei Drittel ihrer Kursbesuche (65\%) erfolgreich abgeschlossen, wobei insgesamt Jugendliche mit Migrationshintergrund erfolgreicher als KundInnen aus Österreich (68 \% vs. $55 \%$ ) waren.

Die Kursbesuche werden mehrheitlich (63\%) als nützlich erlebt, vor allem MigrantInnen empfinden diese nützlicher als ihre österreichischen KollegInnen (66 \% vs. $53 \%)$. Eine besonders hohe Nützlichkeit wird jenen Kursen bescheinigt, die erfolgreich abgeschlossen wurden oder die aus eigenem Wunsch zustanden kamen. Insbesondere Deutsch- und Hauptschulabschlusskurse, Lehr- und Berufsausbildungen sowie mädchenspezifische Maßnahmen werden als sehr nützlich wahrgenommen, während dies auf Zusatzqualifikationen und Berufsorientierungsmaßnahmen deutlich weniger zutrifft.

Mit den verschiedenen Serviceleistungen des Wiener AMS Jugendliche sind insgesamt $85 \%$ der befragten KundInnen zufrieden. Auch der neue Standort, die Verbesserungen beim Terminvereinbarungssystem und die kürzeren Wartezeiten tragen zur hohen Zufriedenheit der Jugendlichen bei. Für die Zukunft wünschen sich die Jugendlichen vom AMS in erster Linie eine Arbeit oder eine Lehrstelle vermittelt zu bekommen, gefolgt von einer höheren finanziellen Unterstützung, einer bessere Beratung und Unterstützung sowie dem Besuch von Kursmaßnahmen. Im Vergleich zur Erhebung im Jahr 2007 hat bei den KundInnen mit Migrationshintergrund der Wunsch nach einer raschen Vermittlung auf einen Arbeits- oder Ausbildungsplatz an Priorität gewonnen, während der Bedarf nach einer besseren Betreuung und angenehmeren Rahmenbedingungen auf Grund der realisierten organisatorischen Veränderungen an Bedeutung verloren hat. 


\section{Handlungs- und Interventionsfelder}

Die Ergebnisse der Evaluierung zeigen, dass das AMS Jugendliche mit den gesetzten Maßnahmen sowohl in Hinblick auf organisatorische Neuerungen als auch im Bereich des Diversity Management auf positive Resonanz der Betroffenen - Jugendliche wie BeraterInnen - stoßen. Dennoch konnten einige Bereiche identifiziert werden, die Platz für Optimierungen bieten.

Im Beratungsprozess selbst gilt es, laufend die Qualität der Beratung zu verbessern. Dazu bedarf es vermehrter Ressourcen und ein Freispielen der BeraterInnen von zurzeit dominierenden administrativen Tätigkeiten, damit sich diese der Klientel uneingeschränkt widmen können. Eine langfristige, kontinuierliche Beratung durch eine Person sowie die verstärkte Möglichkeit der Kontaktnahme zu nachgelagerten Dienstleistern (Kursanbietern, Beratungseinrichtungen) könnte dies zusätzlich fördern.

In Hinblick auf Kursmaßnahmen ist ein Augenmerk sowohl auf die organisatorischen Rahmenbedingungen der Kursgestaltung als auch auf inhaltliche Verbesserungen oder gezielte Änderungen einzelner Maßnahmen zu legen. Ein umfangreicheres, differenzierteres Kursangebot sowie eine flexiblere zeitliche Gestaltung (z. B. mehrere Starttermine) könnte die Passgenauigkeit erhöhen. Bei der TeilnehmerInnenselektion ist dem Creaming vorzubeugen, indem nicht nur quantitative Indikatoren der Erfolgsmessung herangezogen werden. Umfassendere Informationen vor Kursbeginn könnten zur Motivationssteigerung beitragen ebenso wie eine differenzierte Zielgruppenausrichtung (z. B. homogene Lerngruppen entsprechend unterschiedlicher Lernniveaus). Spezielle Kurse für Mädchen oder MigrantInnen sind in Hinblick auf mögliche Diskriminierungen zu untersuchen. Insgesamt ist der „Kurszwang“, um den Übertritt in die Langzeitarbeitslosigkeit zu verhindern, zu hinterfragen, der oftmals Kursbesuche zur Folge hat, die weder in Zusammenhang mit den Berufswünschen der KundInnen noch in Zusammenhang mit bisherigen Qualifizierungsmaßnahmen stehen. Die Konzeption kontinuierlicher Qualifizierungspfade für die KundInnen wäre eine Alternative.

Inhaltlich ist eine Erweiterung der Hauptschulabschlusskurse sowie der FacharbeiterInnenintensivausbildungen auf andere Bereiche notwendig. Deutschkurse sollten auch auf hohem Niveau angeboten werden und bei der Zuteilung das allgemeine Ausbildungsniveau der Jugendlichen berücksichtigt werden. Auch Fachsprachen sollten in speziellen Deutschkursen integriert werden. Das Berufsorientierungscoaching erscheint reformbedürftig. Die Ausbildungslehrgänge müssen verstärkt Vermittlungsaktivitäten und Kooperationen mit der Wirtschaft beinhalten, um die weitere Arbeitsmarktintegration zu erhöhen. Maßnahmen zur Qualitätssicherung der Kurse sind zu setzen, um Qualitätsschwankungen zu vermeiden.

In Hinblick auf die Organisation ist ein besonderes Augenmerk auf den Erstkontakt mit dem AMS zu legen, um diesen friktionsfrei zu gestalten und die KundInnen nicht zu überfordern. In diesem Sinn sollte auch die Homepage durchforstet werden. Innerhalb des AMS ist der Informationsfluss auch abteilungsübergreifend zu erhöhen und unterstützende Maßnahmen für die MitarbeiterInnen (Supervision, Teamsitzungen etc.) zu setzen. Diversity-Aktivitäten in Form von Vorträgen und Lehrgängen sollten ausgebaut und auch MitarbeiterInnen mit derzeit geringem Interesse dazu motiviert werden, diese zu besuchen. Die Ressourcen der MitarbeiterInnen mit Migrationshintergrund könnten über einen internen Know-How Transfer besser genutzt werden. 
Eine intensivere Zusammenarbeit mit der Schule, der Wirtschaft und der Community ist anzustreben. Oftmals werden schulische Probleme an das AMS „weiterdelegiert" und die Jugendlichen verlassen die Schule mit positivem Abschluss aber zu geringen Kenntnissen, um am Arbeitsmarkt Fuß fassen zu können. Hier könnte ein vermehrter Austausch zu einem friktionsfreieren Übergang von der Schule zum AMS führen. Dies betrifft v. a. Berufsorientierungsmaßnahmen, die in der Schule vermehrt gesetzt werden könnten und eine Streuung der Informationen zum AMS. Diese Informationsverbreitung v. a. in Hinblick auf Notwendigkeiten im Zusammenhang mit dem AMS sollte auch vermehrt über MigrantInnenorganisationen stattfinden, um auch die Eltern der Jugendlichen mit einzubinden und deren Informationsstand und Wille zur Zusammenarbeit zu erhöhen.

Um den Vermittlungsprozess zu erleichtern, ist auch eine vermehrte Kooperation mit der Wirtschaft gefragt. Dabei sind mehr Unternehmen zu motivieren, ihre Stellen dem AMS zu melden und den Bewerberlnnen bzw. dem AMS Feedback zu geben. Zudem müssen die Unternehmen verstärkt für die Migrationsthematik sensibilisiert werden und entsprechend beraten und betreut werden, damit (unbewusste) Diskriminierungsmuster nicht greifen. 


\section{Einleitung}

\subsection{Vorbemerkungen}

Personen mit Migrationshintergrund stellen eine bedeutende KlientInnengruppe des Wiener Arbeitsmarktservice (AMS) für Jugendliche dar. Diese Personengruppe ist am Arbeitsmarkt noch immer von Diskriminierung betroffen und weist daher ein erhöhtes Arbeitslosigkeitsrisiko und überdurchschnittliche Arbeitslosigkeitsraten auf. In Wien, dem Bundesland mit dem höchsten MigrantInnenanteil, zeigt sich dieses Arbeitsmarktproblem besonders deutlich. Bei Jugendlichen ist der MigrantInnenanteil höher als in der sonstigen Bevölkerung und auf Grund der Lehrstellenlücke am Lehrstellenmarkt die Situation besonders prekär. Die multiple Problemlage, der sich Jugendliche mit Migrationshintergrund am Arbeitsmarkt gegenüber sehen, führt dazu, dass sie häufig zu KundInnen des Wiener AMS für Jugendliche werden.

Das AMS für Jugendliche in Wien versucht, die Leistungen und Beratungen auf die speziellen Bedürfnisse der jungen Zielgruppe anzupassen und laufend zu optimieren. Ende 2009 erfolgte die Übersiedlung vom Standort Neubaugasse in ein neues Gebäude am Gumpendorfer Gürtel. Dieser Standortwechsel war auch von internen Umstrukturierungsmaßnahmen und organisatorischen Veränderungen begleitet. Auch im Sinne des Diversity Management entwickelt das AMS Jugendliche laufend seine Leistungen für die spezifische Zielgruppe weiter. Um die Angebote weiter zu optimieren und die Neuerungen zu analysieren, bedarf es vertiefender Kenntnisse über die Bedürfnisse und Erwartungshaltungen der migrantischen Zielgruppe. Daher wurde die KMU FORSCHUNG AUSTRIA mit der Evaluierung der Beratung für Jugendliche mit Migrationshintergrund am Wiener AMS Jugendliche beauftragt.

Ziel der Evaluierung ist es, das Beratungsangebot und den Beratungsprozess im AMS Jugendliche zu analysieren und im Sinne des Diversity Management weiterzuentwickeln. Zu diesem Zwecke wurden die organisatorischen Veränderungen des AMS untersucht sowie der Bedarf der Zielgruppe der Jugendlichen mit Migrationshintergrund in Hinblick auf die Beratung erhoben und mögliche Diskrepanzen identifiziert. Zudem wurden die Ergebnisse mit der Studie aus dem Jahr 2007 verglichen, um Entwicklungen im Zeitablauf zu identifizieren. Darauf aufbauend werden Handlungsoptionen aufgezeigt, die zu einer weiteren Optimierung der Beratung im AMS Jugendliche für die spezifische Zielgruppe beitragen können. Folgende Forschungsfragen haben u. a. die Evaluierung geleitet:

- Wie ist der Beratungsprozess im AMS Jugendliche gestaltet? Welche organisatorischen Veränderungen wurden bei den AMS-Beratungen im AMS Jugendliche (für die spezifische Zielgruppe) durchgeführt?

- Welche Erwartungen und Wünsche haben Jugendliche an die AMS Beratung? Gibt es je nach Herkunft Unterschiede?

- Wie werden die Veränderungen am AMS Jugendliche von KundInnen und BeraterInnen wahrgenommen?

- Welche besonderen Probleme treten bei der Beratung der Zielgruppe der MigrantInnen auf (aus BeraterInnen- und KundInnensicht)? 
- Gibt es Diskrepanzen zwischen den angebotenen Beratungen und den Erwartungen der Jugendlichen?

- Wie kann die Beratung am AMS Jugendliche allgemein sowie speziell für Personen mit Migrationshintergrund optimiert und weiterentwickelt werden?

Im vorliegenden Endbericht werden die Ergebnisse der Evaluierung dargestellt. In Kapitel 1.2 findet sich die Beschreibung der angewandten Methodik, Kapitel 2 enthält einige Daten zur potentiellen Zielgruppe und zum Wiener Arbeits- und Lehrstellenmarkt und erläutert die Organisation und Abläufe im AMS Jugendliche. Kapitel 3 beschäftigt sich mit der KundInnenstruktur im Allgemeinen und mit den Charakteristika der Jugendlichen mit Migrationshintergrund in Hinblick auf Integration und Migration im Besonderen. Ausbildung und Arbeitsmarkterfahrungen der Jugendlichen werden in Kapitel 4 beschrieben. Der Beratungsprozess und die Betreuung der Jugendlichen durch das AMS sind Gegenstand von Kapitel 5. In Kapitel 6 werden aus den vorliegenden Ergebnissen Schlussfolgerungen gezogen, in welchen Bereichen die AMS-Beratung für Jugendliche mit Migrationshintergrund verbessert werden kann.

\subsection{Methodik}

\section{Passiv teilnehmende Beobachtung von Beratungsgespräche}

Um den Ablauf eines typischen Beratungsgesprächs näher zu untersuchen und die Auswirkungen der organisatorischen Veränderungen direkt zu erfassen, erfolgte eine offene Beobachtung der Beratungsgespräche, d. h. mit dem Wissen der zu beobachtenden Personen ohne Interaktion mit dem Beratungsprozess. Es wurden an einem Tag rd. 20 Informations- bzw. Beratungsgespräche mit Jugendlichen in der Infozone und der Beratungszone der RGS Jugendliche beobachtet.

\section{Workshops mit AMS-MitarbeiterInnen}

Um den Beratungsprozess aus Sicht des AMS zu analysieren sowie Verbesserungsvorschläge zur Optimierung der Beratungssituation zu erarbeiten, wurden 4 Workshops mit AMS-MitarbeiterInnen aus verschiedenen Zonen durchgeführt:

- ein Workshop mit MitarbeiterInnen des BerufsInfoZentrum (BIZ) und der Infozone,

- ein Workshop mit AMS-BeraterInnen der Servicezone und

- zwei Workshops mit AMS-BeraterInnen der Beratungszone.

Die 4 Workshops fanden im Juli und August 2010 in den Räumlichkeiten der RGS Jugendliche statt. Die Gespräche in den Workshops wurden aufgezeichnet und der Output der Diskussionen u. a. auf Flipcharts festgehalten. Die Auswertung der Workshoptranskripte und Beobachtungsblätter erfolgte entsprechend der grobanalytischen Methode. 


\section{Quantitative Befragung der AMS-KundInnen}

Im Mittelpunkt des Projektes stand die Befragung von Jugendlichen, die im AMS Jugendliche Beratung bzw. Serviceleistungen in Anspruch nehmen. Die Befragung erfolgte in den Räumlichkeiten der Regionalstelle im Zeitraum vom 21. bis 28. Juni 2010. Die Jugendlichen wurden vor bzw. nach ihrem Beratungsgespräch vor Ort persönlich von wissenschaftlichen MitarbeiterInnen und AssistentInnen der KMU FORSCHUNG AUSTRIA befragt. Die Interviewpersonen wurden zufällig gewählt. Der Befragung lag ein standardisierter Fragebogen mit hauptsächlich geschlossenen Fragen zu Grunde.

Ziel war es, insgesamt rd. 400 KundInnen des AMS Jugendliche zu interviewen, wobei rd. 300 Personen mit Migrationshintergrund erreicht werden sollten. Die Befragung von rd. 100 Jugendlichen ohne Migrationshintergrund sollte Aufschluss darüber geben, welche Problembereiche „typisch“ für die Klientel des AMS Jugendliche im Allgemeinen ist und wo es spezifische Herausforderungen in Hinblick auf den Migrationshintergrund gibt.

Personen mit Migrationshintergrund wurden für den Zweck der Studie definiert als Personen, die

- eine ausländische Staatsbürgerschaft besitzen und/oder

- im Ausland geboren sind und/oder

- deren Eltern im Ausland geboren sind und/oder

- deren Umgangssprache mit der Familie auch eine andere Sprache als Deutsch ist.

Der Migrationshintergrund der befragten Jugendlichen wurde anhand mehrerer Faktoren (Staatsbürgerschaft und Geburtsort der Jugendlichen, Geburtsort der Eltern; Sprachen, die innerhalb der Familie (mit Eltern und Geschwister) gesprochen werden) abgefragt bzw. ermittelt.

Insgesamt wurden 431 Personen interviewt, davon 311 Jugendliche mit Migrationshintergrund und 120 Jugendliche ohne Migrationshintergrund (siehe Tabelle 1). Der Anteil an weiblichen Jugendlichen unter allen Befragten lag bei $44 \%$. Ein Drittel der Jugendlichen war zum Befragungszeitpunkt unter 17 Jahre alt und $20 \%$ war 18 Jahre alt. Der Anteil der über 19-Jährigen lag bei $47 \%$.

Tabelle 1 Allgemeine Charakteristika der Stichprobe

\begin{tabular}{l|l|c|c}
\hline Merkmal & Ausprägungen & Anzahl & in Prozent \\
\hline \multirow{2}{*}{ Geschlecht } & männlich & 243 & 56 \\
& weiblich & 188 & 44 \\
\hline \multirow{2}{*}{ Migrationshintergrund } & mit Migrationshintergrund & 311 & 72 \\
& ohne Migrationshintergrund & 120 & 28 \\
\hline \multirow{3}{*}{ Alter } & unter 16 Jahre & 73 & 17 \\
& 17 Jahre & 67 & 16 \\
& 18 Jahre & 87 & 20 \\
& 19 Jahre & 91 & 21 \\
& über 20 Jahre & 113 & 26 \\
\hline \hline
\end{tabular}

Quelle: KMU FORSCHUNG AUSTRIA 


\section{Fokusgruppen mit jugendlichen AMS-KundInnen in Schulungseinrichtungen}

In Ergänzung zur quantitativen Befragung der Jugendlichen wurden 3 Fokusgruppen mit Jugendlichen (vorwiegend mit Migrationshintergrund) durchgeführt. Dabei handelte es sich um moderierte Gruppendiskussionen, denen ein Gesprächsleitfaden zugrunde lag. Das Ziel war es, mehr über die Erlebnisse der Jugendliche mit der AMS-Beratung und den AMS-Kursen zu erfahren sowie ihre Bedürfnisse und Erwartungshaltungen hinsichtlich der AMS-Beratung zu diskutieren. Die Fokusgruppen mit jeweils 4 bis 8 Teilnehmerlnnen fanden bei Schulungsträgern des AMS Jugendliche (bfi Wien - Jugendwerkstatt, Jugend am Werk und Spacelab) im September und Oktober 2010 statt: Insgesamt wurden 17 Jugendliche (11 Buben und 6 Mädchen) im Rahmen der Fokusgruppen befragt.

Die Fokusgruppeninterviews wurden aufgezeichnet, transkribiert und nach der grobanalytische Methode ausgewertet. 


\section{Das Wiener AMS Jugendliche}

\subsection{Jugendarbeitslosigkeit in Wien}

Am Wiener AMS Jugendliche werden alle arbeits- oder lehrstellensuchenden Jugendlichen unter 21 Jahren, die in der Bundeshauptstadt leben, betreut. Im Jahr 2009 waren insgesamt rd. 12.600 jugendliche KundInnen in der Geschäftsstelle für Jugendliche vorgemerkt. Der Großteil der KundInnen, nämlich rd. 5.490 Personen (44\%), befand sich in Schulungen, rd. 4.030 Jugendliche (32\%) waren arbeitslos gemeldet und rd. 2.250 Personen (18\%) wandten sich an das AMS, weil sie eine Lehrstelle suchten. Die rd. 4.030 am AMS Jugendliche als arbeitslos vorgemerkten KundInnen entsprechen beinahe zwei Fünftel aller arbeitslosen Jugendlichen unter 25 Jahre ( $r d$. $39 \%$ ) in Wien. Dieser Anteil ist seit 2005 deutlich gestiegen und weist auf die wachsende Anzahl an jungen Arbeitslosen unter 21 hin.

Das AMS Jugendliche in Wien verzeichnete mit Ausnahme des Jahres 2006 einen kontinuierlichen Anstieg an vorgemerkten arbeitslosen Jugendlichen, wie Tabelle 2 zeigt. Sowohl in Österreich als auch in Wien ist dagegen im Zeitraum von 2006 bis 2008 die Jugendarbeitslosigkeit zurückgegangen, wobei sich 2006 und 2007 österreichweit ein stärkerer Rückgang zeigte als in der Bundeshauptstadt. 2009 machte sich die Wirtschaftskrise mit einem deutlichen Anstieg der Jugendarbeitslosigkeit bemerkbar, wobei Wien - und damit auch das AMS Jugendliche - davon weniger stark betroffen war als Österreich insgesamt (+25,9\%). Der Anteil der Jugendlichen an der Gesamtarbeitslosigkeit in Wien ist mit 14,3\% geringer als in Österreich (16,5\%).

Tabelle 2 Vorgemerkte arbeitslose Jugendliche (15 bis unter 25 Jahren) am AMS Jugendliche, in Wien und Österreich, 2005 bis 2009

\begin{tabular}{|c|c|c|c|c|c|}
\hline & 2005 & 2006 & 2007 & 2008 & 2009 \\
\hline \multicolumn{6}{|c|}{ Arbeitslose Jugendliche am Wiener AMS Jugendliche (Personen unter 21 Jahren) } \\
\hline insgesamt & 3.398 & 3.292 & 3.438 & 3.606 & 4.031 \\
\hline Veränderung zum Vorjahr in \% & $3,9 \%$ & $-3,1 \%$ & $4,4 \%$ & $4,9 \%$ & $11,8 \%$ \\
\hline $\begin{array}{r}\text { Anteil an allen arbeitslosen } \\
\text { Jugendlichen in Wien \% }\end{array}$ & $33,3 \%$ & $33,6 \%$ & $36,5 \%$ & $39,9 \%$ & $38,6 \%$ \\
\hline davon ausländische Jugendliche & 821 & 725 & 713 & 727 & 843 \\
\hline Veränderung zum Vorjahr in \% & $5,6 \%$ & $-11,6 \%$ & $-1,7 \%$ & $2,0 \%$ & $16,0 \%$ \\
\hline \multicolumn{6}{|l|}{ Arbeitslose Jugendliche in Wien } \\
\hline insgesamt & 10.192 & 9.799 & 9.413 & 9.033 & 10.455 \\
\hline Veränderung zum Vorjahr in \% & $9,9 \%$ & $-3,9 \%$ & $-3,9 \%$ & $-4,0 \%$ & $15,7 \%$ \\
\hline Anteil an Gesamtarbeitslosigkeit in \% & $12,5 \%$ & $12,5 \%$ & $13,0 \%$ & $13,6 \%$ & $14,3 \%$ \\
\hline davon ausländische Jugendliche & 2.504 & 2.324 & 2.146 & 1.976 & 2.271 \\
\hline Veränderung zum Vorjahr in $\%$ & $17,1 \%$ & $-7,2 \%$ & $-7,7 \%$ & $-7,9 \%$ & $14,9 \%$ \\
\hline \multicolumn{6}{|l|}{ Arbeitslose Jugendliche in Österreich } \\
\hline insgesamt & 41.568 & 38.095 & 35.140 & 34.069 & 42.908 \\
\hline Veränderung zum Vorjahr in \% & $7,3 \%$ & $-8,4 \%$ & $-7,8 \%$ & $-3,0 \%$ & $25,9 \%$ \\
\hline Anteil an Gesamtarbeitslosigkeit in \% & $16,5 \%$ & $15,9 \%$ & $15,8 \%$ & $16,1 \%$ & $16,5 \%$ \\
\hline davon ausländische Jugendliche & 6.333 & 5.705 & 5.231 & 5.008 & 6.230 \\
\hline Veränderung zum Vorjahr in \% & $14,2 \%$ & $-9,9 \%$ & $-8,3 \%$ & $-4,3 \%$ & $24,4 \%$ \\
\hline
\end{tabular}


Nicht nur die vor allem im Jahr 2009 stark steigende Anzahl arbeitsloser Jugendlicher, sondern auch die angespannte Situation am Lehrstellenmarkt wirkt sich auf die Betreuungssituation beim AMS aus, da auch Lehrstellensuchende betreut werden. Insbesondere in Wien gibt es regelmäßig deutlich mehr Lehrstellensuchende als offene Lehrstellen. In der Bundeshauptstadt standen im Jahr 2009337 offene Lehrstellen 1.419 Lehrstellensuchenden gegenüber. Daraus resultiert eine Lehrstellenandrangsziffer von 4,2, d.h. durchschnittlich konkurrierten etwa vier Jugendliche um eine offene Lehrstelle, während österreichweit weniger als zwei Lehrstellensuchende einer offenen Lehrstelle gegenüber standen (siehe Tabelle 3).

In den Jahren 2006 und 2008 ist das Angebot an offenen Lehrstellen - u. a. bedingt durch Förderungen - sowohl in Wien als auch in ganz Österreich gestiegen. Während in Wien die Anzahl der Lehrstellensuchenden seit 2007 kontinuierlich zurückgeht, macht sich österreichweit nach einem starken Rückgang 2007 wieder ein Anstieg an InteressentInnen bemerkbar.

Tabelle 3 Lehrstellenmarkt in Wien und Österreich, 2005 bis 2009

\begin{tabular}{l|c|c|c|c|c|c}
\hline & \multicolumn{3}{|c|}{ Wien } & \multicolumn{3}{c}{ Österreich } \\
\hline & $\begin{array}{c}\text { Offene } \\
\text { Lehrstellen* }\end{array}$ & $\begin{array}{c}\text { Lehrstellen } \\
\text { suchende* }\end{array}$ & $\begin{array}{c}\text { Lehrstellen- } \\
\text { andrangsziffer }\end{array}$ & $\begin{array}{c}\text { Offene } \\
\text { Lehrstellen* }\end{array}$ & $\begin{array}{c}\text { Lehrstellen } \\
\text { suchende* }\end{array}$ & $\begin{array}{c}\text { Lehrstellen- } \\
\text { andrangsziffer }\end{array}$ \\
\hline 2005 & 283 & 1.741 & 6,2 & 2.900 & 6.156 & 2,1 \\
\hline 2006 & 449 & 1.790 & 4,0 & 3.611 & 6.099 & 1,7 \\
\hline 2007 & 386 & 1.663 & 4,3 & 3.546 & 5.689 & 1,6 \\
\hline 2008 & 414 & 1.660 & 4,0 & 3.633 & 5.695 & 1,6 \\
\hline 2009 & 337 & 1.419 & 4,2 & 3.279 & 5.944 & 1,8 \\
\hline
\end{tabular}

*sofort verfügbare offene Lehrstellen bzw. Lehrstellensuchende

Quelle: Arbeitsmarktservice (AMS)

\subsection{Organisation}

Das Wiener AMS Jugendliche ist im November 2009 vom Standort Neubaugasse in einen neues, deutlich größeres Haus am Gumpendorfer Gürtel gezogen. Die Übersiedlung war von internen Umstrukturierungsprozessen begleitet, es wurden u. a. die Kundengruppen zwischen den AMS-Zonen neu aufgeteilt und ein neues Terminvergabesystem implementiert, um die Spontanvorsprachen der KundInnen besser steuern und dadurch auch die Jugendlichen effizienter betreuen zu können. Eine weitere Neuerung ist die im Februar 2009 ins Leben gerufene Schulaustreterlnnen-Gruppe (SAG), die allen Jugendlichen, die erstmalig von der Schule in den Arbeitsmarkt einsteigen wollen, berät und eine individuelle Betreuung der Personen im letzten Schuljahr bis unmittelbar nach Schulaustritt ermöglicht. 
Die einzelnen AMS-Zonen sind $u$. a. mit folgenden Aufgaben betraut:

Infozone: Die erste Anlaufstelle beim AMS stellt für die Jugendlichen das Info-Pult dar, wo sie erste grundlegende Informationen über das AMS und Termine - bei einem Erstbesuch in der Infozone Backoffice bzw. bei Spontanvorsprachen von bestehenden KundInnen bei ihrem/r Beraterln - erhalten. Im Falle einer Erstvorsprache erfolgt in der Infozone Backoffice die Abklärung zum schulischen Hintergrund, über berufliche Erfahrung und Berufswünsche, die der/die Jugendliche mitbringt, welche Unterstützung er/sie benötigt und ob Anspruch auf Leistungsbezug besteht. Anschließend werden die weiterführenden Termine bei den Beraterlnnen, die meist in vier bis sechs Wochen stattfinden, vereinbart. Werden diese Beratungstermine nicht eingehalten, wird der/die Kundln abgebucht und muss sich bei der nächsten Vorsprache wieder in der Infozone melden. Ist der/die Jugendliche bei seinem Erstbesuch in der Infozone Backoffice beruflich noch orientierungslos, wird er/sie an das BerufsInfoZentrum (BIZ) verwiesen.

Servicezone: Vor der Umstrukturierungsphase wurden in dieser Zone nahezu alle AMS-KundInnen (mit gewissen Ausnahmen) während der ersten 3 Monate ihrer Vormerkung betreut, nun sind die BeraterInnen in dieser Zone nur für Personen zuständig, die direkt am Arbeitsmarkt vermittelbar sind, da sie z. B. bereits über eine abgeschlossene Ausbildung verfügen. Im Kundenkontakt steht daher die unmittelbare Vermittlung auf Arbeitsplätze im Vordergrund, es werden aber auch Kurse vermittelt, um Übertritte in die Langzeitarbeitslosigkeit zu vermeiden. Die Zielgruppe wird so lange in der Servicezone betreut, bis sie einen Arbeitsplatz findet oder auf Grund der Erreichung der Altersgrenze von 21 Jahren in eine andere Geschäftsstelle überstellt werden muss. Ein Wechsel der KundInnen zwischen Service- und Beratungszone erfolgt nur mehr dann, wenn sich die Zielgruppenzugehörigkeit der KundInnen ändert (z. B. durch einen Ausbildungsabschluss oder einen Reha-Fall). In der Servicezone werden alle finanziellen und versicherungstechnischen Angelegenheiten (Bezug von Arbeitslosengeld, Notstandshilfe, Versicherungsschutz, etc.) geregelt.

Beratungszone: Die BeraterInnen in dieser Zone betreuen Kundlnnen, die noch nicht ,job-ready" sind, das sind alle Jugendlichen, die keine Ausbildung absolviert haben, Vermittlungsprobleme aufweisen oder zu einer Problemgruppe (wie z. B. Personen in der Rehabilitation, Drogensüchtige) zählen. Diese Zielgruppen benötigen eine intensivere Betreuung und Hilfe bei der Arbeitssuche. Neben der Vermittlung in Arbeitsverhältnisse und Lehrstellen zählen die berufliche Beratung sowie die Vermittlung in Kurse zu den zentralen Aufgaben der Beratungszone. Die Auswahl der Kurse erfolgt unter der Berücksichtigung der Voraussetzungen, die die KundInnen mitbringen, wobei häufig Deutschkurse, Hauptschulabschlusskurse, Berufsorientierungsmaßnahmen und überbetriebliche Lehrausbildungen vermittelt werden. Die Beratungsgespräche finden nur mehr zu einem konkreten Termin statt, Spontanvorsprachen, die früher sehr häufig waren, werden bereits in der Infozone, wo konkrete Termine vereinbart werden, abgefangen. Trotz Erhöhung der personellen Ressourcen betreut ein/e Berater/in durchschnittlich rd. 600 KundInnen.

BerufsInfoZentrum (BIZ): Eines der fünf Wiener BerufsInfoZentren, die Bildungsberatung und Berufsinformation anbieten, ist im AMS für Jugendliche angesiedelt. Hier können sich die Jugendlichen über Berufe, Beschäftigungsmöglichkeiten, Jobchancen sowie Aus- und Weiterbildungsmöglichkeiten informieren. Den KundInnen steht eine Fülle an Informationsmaterial in Form von Broschüren, Infomappen, Videofilmen und PCs zur Verfügung bzw. können sie auch ein Beratungsgespräch mit einem/r BIZ-Mitarbeiterln führen. Neben Einzelgesprächen mit Jugendlichen, die terminlich möglichst unbürokratisch gestaltet werden, um dem Einzelnen weiterzuhelfen, werden auch Führungen und Vorträge für Schulklassen veranstaltet. 
Psychologische Abklärung: Ist bei den Jugendlichen aus Sicht der BeraterInnen ein Bedarf nach einer Leistungsabklärung gegeben oder liegt eine besondere Orientierungslosigkeit vor, kann eine genauere psychologische Abklärung erfolgen. Dabei werden ausführliche Anamnesegespräche mit den Jugendlichen und den Eltern geführt sowie psychologische Tests und Interessenstests zur Abklärung der beruflichen Möglichkeiten und Wünsche vorgegeben. Auf Basis dieser Befunde werden Gutachten und Empfehlungen für die weitere berufliche Laufbahn formuliert.

Das neue AMS-Gebäude am Gumpendorfer Gürtel stellt sowohl aus Sicht der AMSBeraterInnen als auch der Jugendlichen eine wesentliche Verbesserung dar. Für die AMS-BeraterInnen haben sich durch den Umzug ihre Arbeits- und Beratungsbedingungen deutlich gebessert und sie können den KundInnen eine angenehmere Beratungsatmosphäre bieten. $75 \%$ der befragten Jugendlichen ist noch der Standort in der Neubaugasse bekannt, daher können sie Vergleiche zwischen dem alten AMS-Gebäude und dem aktuellen Standort am Gumpendorfer Gürtel ziehen. 85 \% dieser KundInnen empfinden die Atmosphäre und die Gestaltung der Innenräume besser als in der Neubaugasse, wie Grafik 1 zeigt. Sie beschreiben den neuen Standort als besser, größer, sauberer, schöner und moderner. Die AMS-MitarbeiterInnen berichten, dass einzelne Jugendliche sogar Angst hatten, dass alte AMS-Gebäude in der Neubaugasse zu betreten.

Aber nicht nur die Räumlichkeiten und die Atmosphäre haben sich verbessert, sondern rd. drei Viertel $(76 \%)$ der befragten AMS-KundInnen fällt auch die Orientierung im Haus leichter und für $69 \%$ ist der Gumpendorfer Gürtel einfacher zu erreichen. Die AMS-BeraterInnen empfinden die Infrastruktur, wie z. B. die größere Infozone, im neuen, größeren Haus wesentlich besser und mehr als zwei Drittel der Jugendlichen schätzen die erweiterten Angebote zur Selbstbedienung (Computer, etc.). Auch die Aufzüge sowie die neue räumliche Einteilung werden von den KundInnen lobend erwähnt.

Grafik 1 Beurteilung des aktuellen Standorts (Gumpendorfer Gürtel) im Vergleich zum alten Standort (Neubaugasse), Anteil der befragten Jugendlichen, die beide Standorte kennen, in Prozent

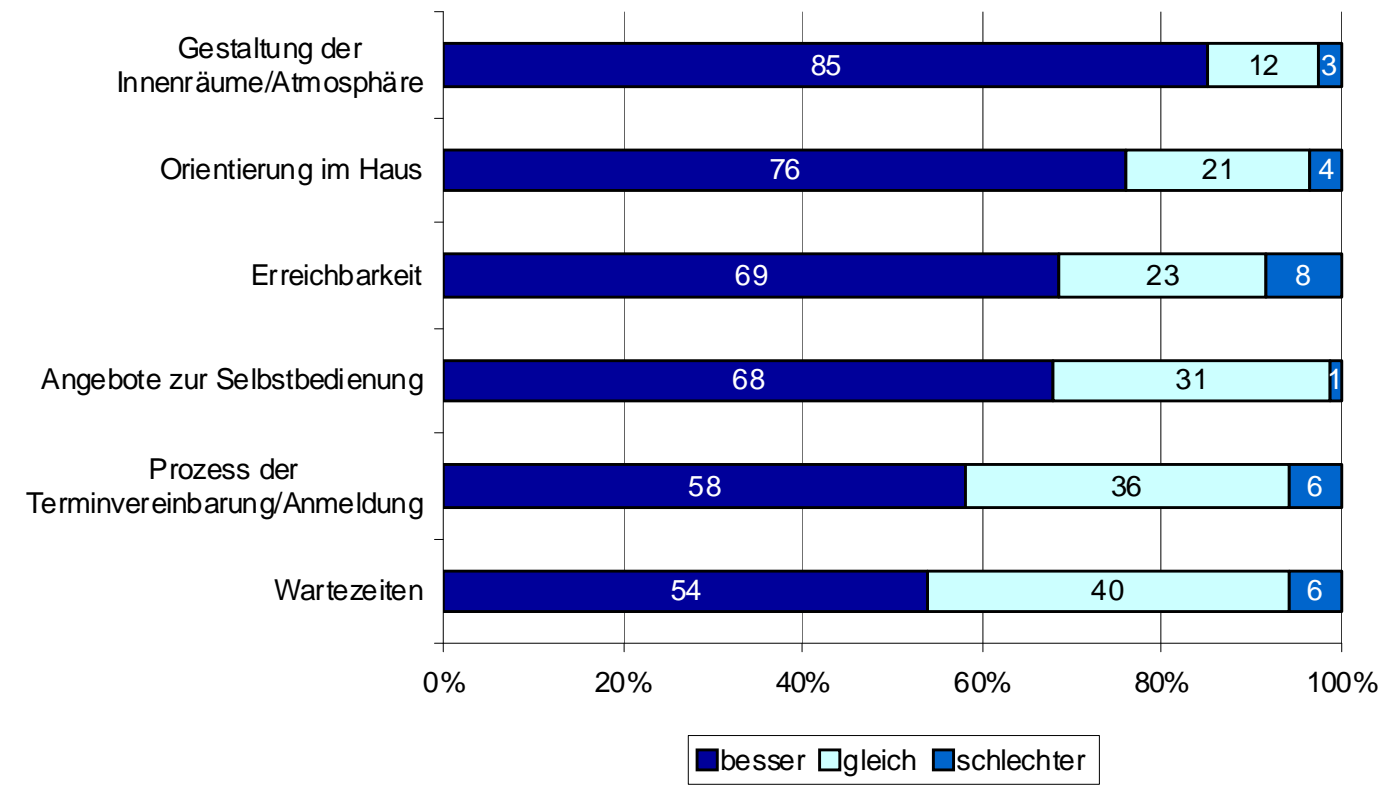

Quelle: KMU FORSCHUNG AUSTRIA, Befragung der jugendlichen AMS-KundInnen, n = 319 - 228 
Im Rahmen der Übersiedlung wurde $u$. a. der Prozess der Terminvereinbarung bzw. das Anmeldeprozedere reformiert. Die Terminvereinbarungen werden nun großteils über die Infozone abgewickelt und die KundInnen müssen keine Nummern mehr ziehen. Die BeraterInnen können sich somit auf eine fixe Anzahl an KundInnenvorsprachen pro Tag einstellen und auf die geplanten Gespräche vorbereiten. In der Neubaugasse traf der Großteil der KundInnen am späten Vormittag beim AMS ein, was für die BeraterInnen belastend war und ihre Arbeitszeit erheblich verlängerte, wie eine Mitarbeiterin folgendermaßen schildert: „Dann waren um 11:15 Uhr noch 7 bis 8 Kunden zu bearbeiten, dann ist man bis 12:30 Uhr gesessen, das längste war 13:15 Uhr und um 13:30 Uhr sind die nächsten Kunden gekommen. Das gibt es jetzt nicht mehr, um 11:15 sind noch 3 Kunden da und dann ist Schluss. Wenn sie ihren Termin nicht einhalten, dann müssen sie zu einem anderen Termin kommen. Das ist perfekt. Das ist eine tausendfache Verbesserung." Auch für $58 \%$ der befragten jugendlichen AMSKundInnen hat sich der Prozess der Terminvereinbarung bzw. Anmeldung verbessert.

Ziel des neuen Terminvereinbarungssystems war, die Wartezeiten für die Kundlnnen zu reduzieren. Wenn der/die KundIn bereits einen fixen Termin hat, fällt kaum mehr eine Wartezeit an. Dies kann aber die BeraterInnen in Zeitdruck bringen, wenn sie sich kurzfristig für eine/n Jugendliche/n mehr Zeit nehmen wollen, da die KundInnen nun erwarten, dass ihre Termine pünktlich eingehalten werden. Nur bei Spontanvorsprachen können noch Wartezeiten entstehen, je nachdem wann noch Termine bei den BeraterInnen verfügbar sind. So kann es vorkommen, dass der/die Jugendliche nicht immer zu seinem/r zuständigen Beraterln kommt, sondern einem/r anderen Beraterln zugewiesen wird, wenn diese/r früher einen Termin frei hat. Dies beeinträchtigt zwar die Betreuungskontinuität, kann sich aber auch positiv auswirken, da ein/e andere/r Beraterln möglicherweise einen anderen Zugang hat und neue Ideen einbringen kann. Aus Sicht der betroffenen KundInnen hat sich für mehr als die Hälfte (54 \%) die Wartezeit verbessert. Aus Sicht der Jugendlichen zeigt sich der neue Standort so: „Am Gumpendorfer Gürtel ist es besser, schöner und es ist nicht mehr so viel los. In der Neubaugasse waren immer ur-viele Leute, das war eine Katastrophe. Die Wartezeiten sind besser geworden." $40 \%$ nehmen jedoch - u. a. bedingt durch häufiges spontanes Erscheinen beim AMS und damit wieder auch längeren Wartezeiten - keine Veränderung wahr.

Das neue Terminvereinbarungssystem hatte auch zum Ziel, die Spontanvorsprachen zu reduzieren. Dies hat sich aus Sicht der AMS-BeraterInnen jedoch nicht erfüllt, denn die Spontanvorsprachen haben sich nur von der Beratungs-/Servicezone in die Infozone verlagert. Dort wird es als sehr problematisch erlebt, dass viele Jugendliche ihre Termine nicht einhalten oder vergessen. Da viele KundInnen noch über keinen Leistungsanspruch verfügen, hat ein Terminversäumnis keine finanziellen Konsequenzen. Außerdem erhalten die meisten Jugendlichen noch am selben Tag einen Termin. Wenn sie häufig ihre Beratungstermine versäumen, hat dies aber zur Folge, dass sie mehrmals die Infozone aufsuchen müssen, bevor sie in die Service- oder Beratungszone weitervermittelt werden können und somit wertvolle Zeit ohne produktive Arbeit mit den KundInnen verstreicht.

Eine weitere organisatorische Neuerung betrifft die Aufteilung der Zielgruppen zwischen Service- und Beratungszone. Diese wird von den AMS-MitarbeiterInnen großteils positiv erlebt. Insbesondere die MitarbeiterInnen der Servicezone sind froh, dass sie sich auf die direkt vermittelbaren KundInnen konzentrieren können. Dadurch ist ihr Beratungsaufwand geringer, sie sind aber zusätzlich mit der gesamten Abwicklung des Leistungsbezugs betraut. Auch die Möglichkeit, die Jugendlichen nach drei Monaten weiterbetreuen zu können, wird sehr geschätzt. Jedoch könnte die Zuteilung der Kun- 
dInnen, die noch nicht unmittelbar am Arbeitsmarkt zu vermitteln sind, in der Beratungszone zu dem Eindruck führen, dass alle Jugendlichen „schwierig“ sind und im Berufsleben nicht Fuß fassen können oder wollen. Auch die Erlebnisse erfolgreicher Vermittlungen finden in der Beratungszone auf Grund der KundInneneinteilung seltener statt als in der Servicezone.

Darüber hinaus werden die Kommunikationsmöglichkeiten zwischen KundInnen und BeraterInnen laufend erweitert. Neben persönlichen Vorsprachen können die Arbeitsuchenden auch per Telefon und E-Mail mit ihrem/r Beraterln in Kontakt treten und ein persönliches e-AMS-Konto anlegen, wo sie u. a. ihre Stelleninserate bearbeiten und die Betreuungsvereinbarung nachlesen können. Dies bringt jedoch auch mehr Arbeitsaufwand für die AMS-BeraterInnen mit sich, da sich vor allem das Beantworten der zahlreichen E-Mails als sehr zeitaufwändig erweist.

\subsection{Diversity Management im Rahmen des Wiener AMS Jugendliche}

Am Wiener AMS Jugendliche wird auf Diversity Management großer Wert gelegt und es fließt als Querschnittsmaterie in alle Kernprozesse mit ein. Ein wesentliches Handlungsfeld der Diversity Management Gruppe bezieht sich auf Informationsaktivitäten für Jugendliche und deren Eltern. In diesem Zusammenhang wurde mehrsprachiges Informationsmaterial entwickelt und einige Formulare und Broschüren auf Türkisch und Serbisch bzw. Kroatisch übersetzt. Darüber hinaus erfolgten Beratungs- und Informationsgespräche in Moscheen und Kulturvereinen in Kooperation mit dem Beratungszentrum für MigrantInnen. Auch an einer Info-DVD zur Gestaltung des Übergangs Schule / Beruf und zum Thema Lehrberufe und Arbeitsmarkt wird gearbeitet, um in erster Linie die migrantischen Eltern über die Lehrausbildung aufzuklären, da diese oftmals keinen Zugang zu diesen Informationen haben. Des Weiteren können die Kooperationen der AMS Landesgeschäftsstelle Wien mit einer türkischen und einer serbischen Zeitung genutzt werden, um die Zielgruppe regelmäßig zu informieren.

Das zweite Handlungsfeld im Bereich des Diversity Managements konzentriert sich auf die Schaffung geeigneter Rahmenbedingungen in der Beratungssituation. Daher wurden Schulungen zur interkulturellen Kommunikation für AMS-MitarbeiterInnen angeboten und verpflichtende Seminare zu Gender- und Diversity-Fragen für Führungskräfte des AMS Wien eingeführt (ab 2011 verpflichtend für alle MitarbeiterInnen). Auch Türkischkurse für AMS-BeraterInnen und Veranstaltungen mit VertreterInnen aus verschiedenen Kulturen oder Einrichtungen stehen regelmäßig auf dem Weiterbildungsprogramm. Darüber hinaus wurden verstärkt neue MitarbeiterInnen mit Migrationshintergrund aufgenommen.

Diversity Management spielt auch bei der Umsetzung von AMS-Qualifizierungsmaßnahmen als drittes Handlungsfeld eine bedeutende Rolle. Für TrainerInnen von AMSMaßnahmen wird z. B. ein Diversitätstraining vorausgesetzt und bei Schulungen für Teilnehmerlnnen werden Module zur interkulturellen Kommunikation integriert. Diversity Management findet auch bei der Entwicklung und Implementierung von MentoringKonzepten, wobei beispielsweise auch Berufsorientierungsmaßnahmen mit Mentoring kombiniert werden, sowie bei der Entwicklung von Pilotmaßnahmen Berücksichtigung.

Um auf die Bedeutung des Diversity Management Themas hinzuweisen und Kooperationen aufzubauen, veranstaltet das AMS Wien Workshops und Tagungen. Außerdem beteiligt es sich aktiv an Veranstaltungen, die sich an UnternehmerInnen mit Migrationshintergrund richten. 
In Zusammenhang mit Diversity-Management stehen für die AMS-BeraterInnen die Qualifizierungsmaßnahmen, die speziell auf die Zielgruppe der Jugendlichen mit Migrationshintergrund zugeschnitten sind, im Vordergrund. Die Beraterlnnen nennen zahlreiche Kursmaßnahmen für MigrantInnen, wie beispielsweise das vielfältige Angebot an Deutschkursen und Alphabetisierungsmaßnahmen. Spezifische Qualifizierungen, wie z. B. die Ausbildung zur Ordinationshilfe oder zur Kindergartenhelferin, richten sich ausschließlich an Mädchen. Dies führt jedoch gleichzeitig zu einer Diskriminierung von Burschen, die sich auch für eine Ausbildung in diesem Bereich interessieren, und kann laut Erfahrung der AMS-Beraterlnnen bei den ausgeschlossenen Kundengruppen zu Ärger und Unverständnis führen. Bei diesen spezifischen Qualifizierungsmaßnahmen sind oftmals Deutschkurse integriert, jedoch könnte ein Deutschkurs auch begleitend zu einer regulären Ausbildung besucht werden. Darüber hinaus merken die AMS-MitarbeiterInnen an, dass bei den speziellen Qualifizierungsangeboten für MigrantInnen auch Kontakte zur Unternehmen geknüpft werden sollten, da sich trotz entsprechender Ausbildung oftmals die Vermittlung z. B. mit Kopftuch und nicht perfekten Deutschkenntnissen schwierig gestaltet. Ein weiteres Angebot für die Zielgruppe sind spezifische Beratungseinrichtungen, wie z. B. das Beratungszentrum für MigrantInnen oder Migln, die den KundInnen die Möglichkeit bieten, Fragen in Zusammenhang mit ihrer Immigration zu beantworten und auch private Probleme zu lösen.

Zu den Diversity-Maßnahmen zählen auch die Informationsaktivitäten des AMS für die Zielgruppe. Mehr als die Hälfte der befragten Jugendlichen (56\%) nehmen diese in Anspruch, wobei sie in erster Linie auf die AMS-Homepage zugreifen. Broschüren oder schriftliches Informationsmaterial werden von den jugendlichen AMS-KundInnen viel seltener als Informationsquelle genutzt. Auch die AMS-BeraterInnen bestätigen, dass die jugendlichen AMS-KundInnen (mit und ohne Migrationshintergrund) kaum schriftliche Informationen (Broschüren, Infoblätter) in Anspruch nehmen. Als Gründe für die mangelnde Nutzung nennen rd. $44 \%$ der Jugendlichen, die nicht auf die Informationsmaterialien des AMS zurückgreifen, dass sie kein Interesse haben bzw. keinen Bedarf dafür sehen. Einigen fehlt auch die Zeit, sich damit zu befassen. Ein Teil der Jugendlichen ist noch nicht auf die Informationsmaterialien aufmerksam geworden oder hat sich diesen noch nie aktiv zugewandt. Aber auch sprachliche Barrieren und Verständigungsprobleme sowie eine nicht ansprechende Aufbereitung (unübersichtlich, uninteressant) werden vereinzelt als Hemmnisse genannt ebenso wie, dass die Materialien als nicht hilfreich erlebt werden.

Auf Schwierigkeiten beim Verstehen des Informationsmaterials weisen auch $9 \%$ der befragten Jugendlichen, die die Materialien des AMS nutzen, hin. Insbesondere Jugendliche mit Migrationshintergrund (11\%) haben auf Grund von Problemen mit der deutschen Sprache eher Schwierigkeiten, das Informationsmaterial zu verstehen, aber auch für rd. $3 \%$ der AMS-KundInnen ohne Migrationshintergrund sind die Angebote nicht gut verständlich. Die KundInnen haben den Eindruck, dass die Broschüren zu kompliziert geschrieben sind. Fremdsprachige Informationsangebote helfen daher aus Sicht der AMS-BeraterInnen nur bedingt, um diesen Verständigungsproblemen entgegenzuwirken. Fremdsprachiges Material erscheint nur für Grundinformationen über die Leistungen des AMS sinnvoll, damit keine sprachlichen Missverständnisse mit KundInnen auftreten. Weiterführende Information über Berufe oder Ausbildungsmöglichkeiten in der Muttersprache anzubieten, wird als weniger zielführend erachtet, da auch die Qualifizierungsmaßnahmen auf Deutsch zu absolvieren sind. Außerdem beobachten die AMS-MitarbeiterInnen, dass einige KundInnen mit Informationsmaterialien in ihrer Muttersprache überfordert sind und diese nicht lesen können, da sie ihre 
Muttersprache meist nur mündlich erlernt haben und teilweise nur regionale Dialekte beherrschen. Daher ist der Bedarf an weiterem fremdsprachigem Informationsmaterial aus Sicht der AMS-MitarbeiterInnen gering.

Das bereits zur Verfügung stehende Informationsmaterial findet bei der Zielgruppe jedoch einen guten Anklang. Mehr als $90 \%$ der befragten Jugendlichen, die schon Materialien des AMS (Broschüren, Internet) in Anspruch genommen haben, gefallen diese (sehr) gut, wie aus Grafik 2 ersichtlich. Lediglich $6 \%$ sprechen diese weniger oder gar nicht an.

Grafik 2 Attraktivität des Informationsmaterials, Anteil der befragten Jugendlichen, die das Material nutzen, in Prozent

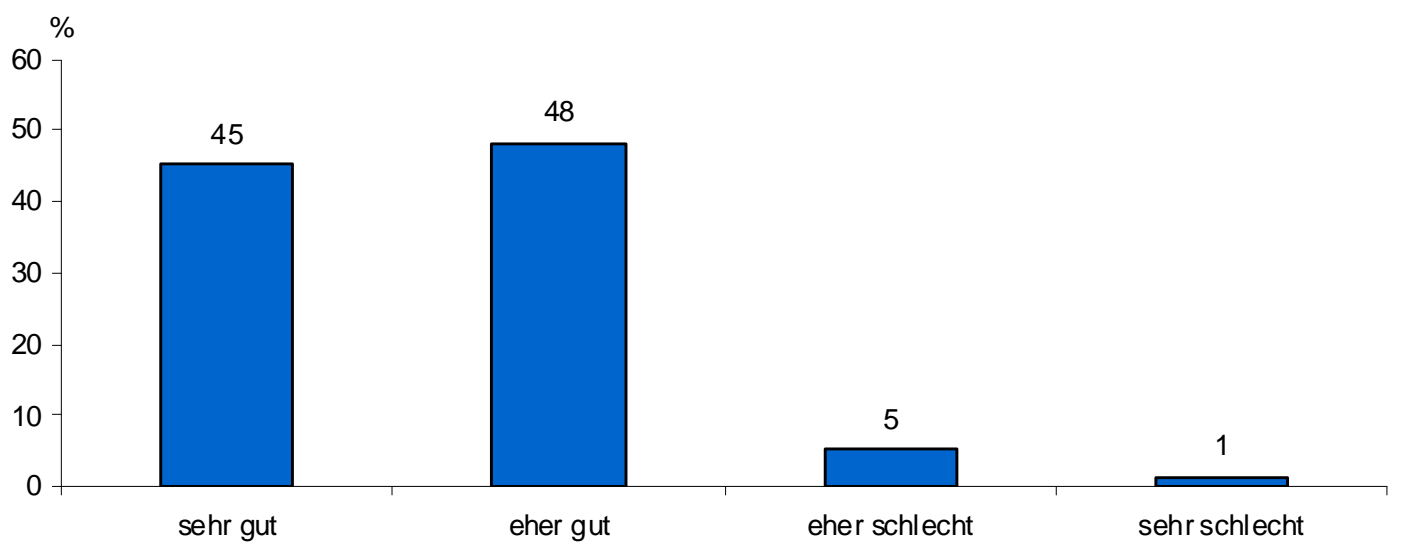

Quelle: KMU FORSCHUNG AUSTRIA, Befragung der jugendlichen AMS-KundInnen, $\mathrm{n}=230$

Verbesserungen des Informationsangebots sind jedoch der Mehrheit der befragten AMS-KundInnen (58\%), die bereits davon Gebrauch machten, nicht aufgefallen bzw. konnten sie diese nicht beurteilen (siehe Grafik 3). $22 \%$ sind Verbesserungen ins Auge gestochen, wobei ihrer Meinung nach die Informationen auf den Broschüren und vor allem im Internet aktueller sind, die Bedienung leichter fällt sowie mehr Auskünfte über Berufe und Stellenangebote enthalten sind. Jedoch hatte auch ein Fünftel den Eindruck, dass sich das Informationsmaterial nicht verbessert hat und im Wesentlichen gleichgeblieben ist.

Grafik 3 Wahrgenommene Verbesserung des Informationsmaterials, Anteil der befragten Jugendlichen, die das Material nutzen, in Prozent

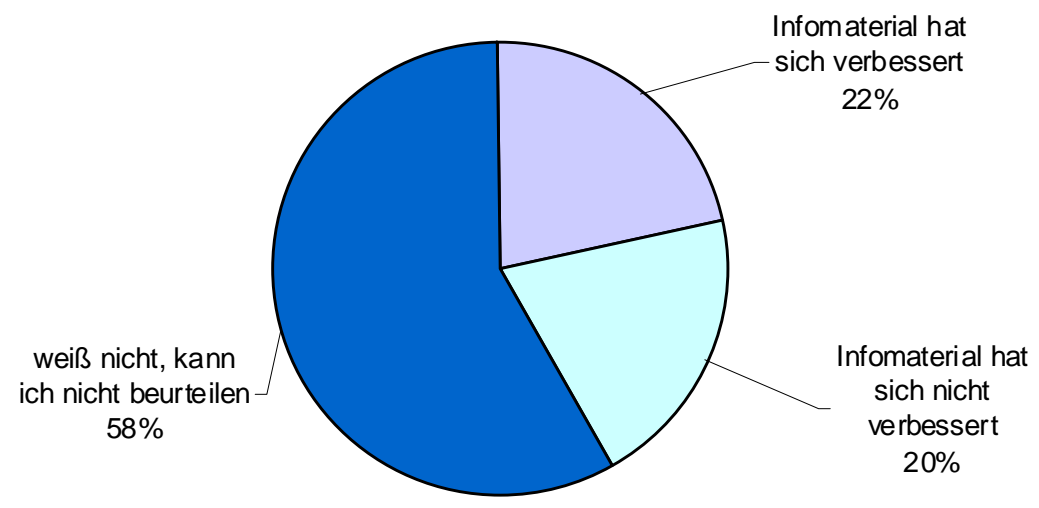

Quelle: KMU FORSCHUNG AUSTRIA, Befragung der jugendlichen AMS-KundInnen, n=199 
In Zusammenhang mit dem weiteren Diversity-Management Handlungsfeld, das sich auf die Schaffung geeigneter Rahmenbedingungen in der Beratungssituation konzentriert, nehmen die AMS-BeraterInnen in erster Linie die steigende Anzahl an MitarbeiterInnen mit Migrationshintergrund wahr. Die Sprachkenntnisse werden - im Gegensatz zu früher - als bedeutende Ressource erkannt. Daher werden gezielt Personen gesucht, die verschiedene Sprachen beherrschen. Eine Beraterin mit Migrationshintergrund berichtet, dass sie dennoch die Kundengespräche auf Deutsch führt und nur im Bedarfsfall ein paar Schlüsselwörter in ihrer Muttersprache einwirft. Sonst besteht die Gefahr, dass zu viele Jugendliche zu ihr kommen und das Beratungsgespräch ausschließlich in der Muttersprache führen wollen. Die KundInnen selbst reagieren meist überrascht auf die fremdsprachige Auskunft und interessieren sich oft für den persönlichen Werdegang der Beraterin. Problematisch ist jedoch, wenn die Jugendlichen nur regionale Dialekte ihrer Muttersprache beherrschen und nicht einmal die Übersetzungen verstehen.

Das gesamte Beratungsgespräch wird jedoch nur sehr selten in einer anderen Sprache abgewickelt. Lediglich 1,7 \% der befragten Jugendlichen mit Migrationshintergrund haben bisher beim AMS ein Beratungsgespräch in einer anderen Sprache als auf Deutsch geführt. Jedoch fände es grundsätzlich mehr als ein Drittel der Befragten (36 \%) sinnvoll, wenn es die Möglichkeit gäbe, die Beratungsgespräche in einer anderen Sprache als auf Deutsch zu führen. Naturgemäß können sich vor allem Personen mit Migrationshintergrund (38\%) für ein muttersprachliches Beratungsangebot begeistern, aber auch rd. $30 \%$ der österreichischen Jugendlichen halten eine solche Zusatzleistung für sinnvoll.

Im Rahmen der Beratungsgespräche beim AMS überwiegt von Seiten der befragten Jugendlichen der Eindruck, dass KundInnen mit und ohne Migrationshintergrund gleich behandelt werden. Dennoch beobachten rd. $16 \%$ aller Jugendlichen, dass MigrantInnen beim AMS eine andere Behandlung widerfahren. Dies trifft vor allem auf Personen mit Migrationshintergrund (18\%) zu wie Grafik 4 zeigt. Die Betroffenen haben vorwiegend das Gefühl, dass sie von den Beraterlnnen schlechter und unfreundlicher behandelt werden und sie fühlen sich weniger verstanden, obwohl sie sich ihrer Meinung nach verständlich ausdrücken. Einzelne MigrantInnen nehmen jedoch auch eine bessere Behandlung wahr, wobei innen mehr Information und Unterstützung geboten wird, da sie am Arbeitsmarkt mehr Schwierigkeiten haben. Diesen Eindruck hat auch etwas mehr als ein Zehntel der befragten AMS-KundInnen ohne Migrationshintergrund (11\%). Diese Jugendlichen haben tendenziell das Gefühl, dass MigrantInnen bevorzugt bzw. besser behandelt werden und leichter zu Unterstützungsangeboten gelangen. Dieser Eindruck kann möglicherweise auch dadurch entstehen, dass sich einzelne Kurse und Beratungsangebote des AMS speziell auf die Zielgruppe der MigrantInnen konzentrieren. 
Grafik 4 Subjektive Wahrnehmung der Behandlung der Jugendlichen mit Migrationshintergrund seitens der AMS-BeraterInnen, Anteil der befragten Jugendlichen nach Migrationshintergrund in Prozent

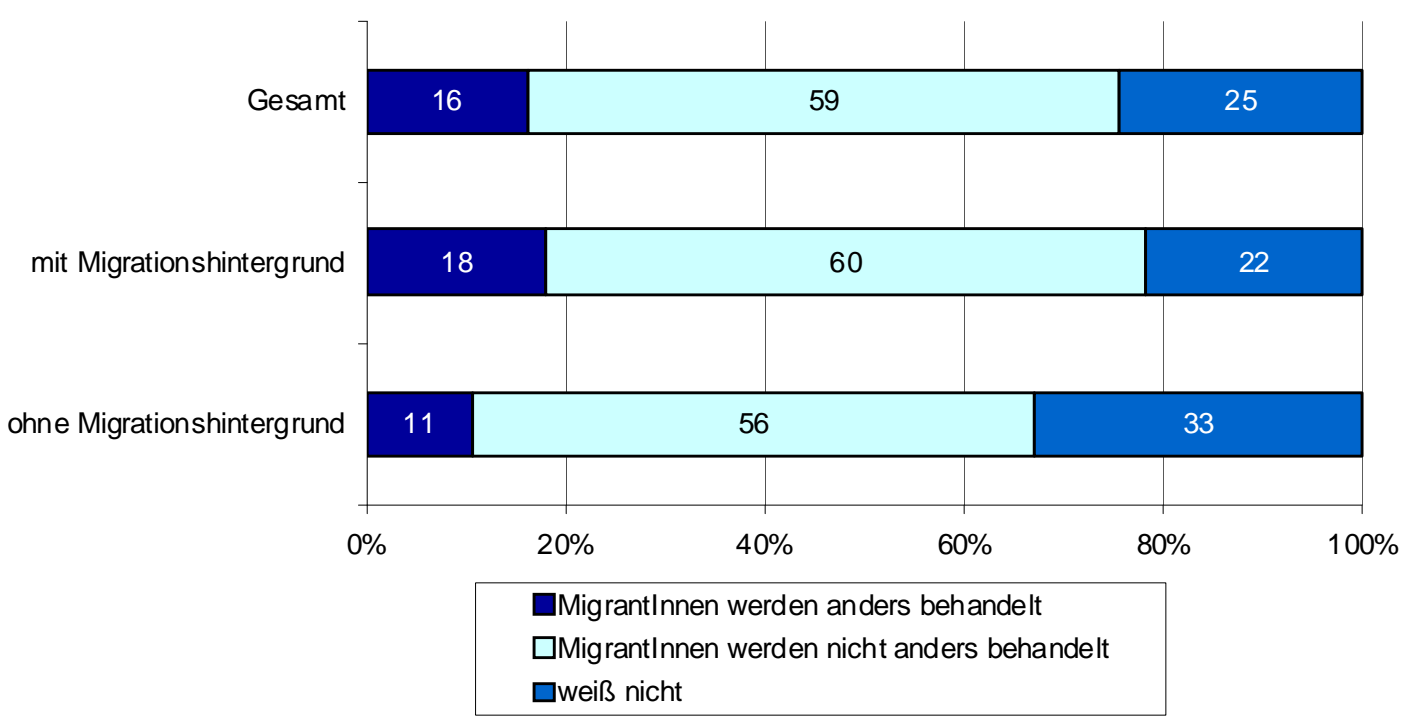

Quelle: KMU FORSCHUNG AUSTRIA, Befragung der jugendlichen AMS-KundInnen, $\mathrm{n}=355$

Als weitere Diversity-Aktivitäten werden Weiterbildungsmaßnahmen für AMS-MitarbeiterInnen, die sich mit dem Thema Diversity Management auseinander setzen, erwähnt. Einige der befragten AMS-BeraterInnen haben bereits an Vorträgen oder Lehrgängen (z.B. SIQUA-Lehrgang) zu diesem Thema teilgenommen. Diese sind auf sehr positive Resonanz gestoßen, da sie den TeilnehmerInnen einen Einblick in das Leben von MigrantInnen und deren Probleme bei einem Eintritt in ein neues Lebensumfeld anschaulich vermittelt haben. Die BeraterInnen würden sich in Zukunft weitere interessante Vorträge zu diesem Thema und eine intensivere Vernetzung mit den Communities wünschen. Auch die für das Thema Diversity-Management zuständigen Personen werden als sehr engagiert und als unterstützend (z. B. in Konfliktfällen) erlebt. Das Angebot an Sprachkursen für AMS-BeraterInnen (z. B. Türkisch-Kurse) wird hingegen als weniger nützlich erachtet, da es kaum möglich ist, mit einem geringen Zeitaufwand eine weitere Fremdsprache zu erlernen und die KundInnen über einen sehr vielfältigen sprachlichen Hintergrund verfügen. Außerdem ist der Bedarf nach zusätzlichen Sprachkenntnissen gering, da Personen mit unzureichenden Deutsch-Kenntnissen zumeist von einem/r Dolmetscher/in aus dem Verwandten- oder Bekanntenkreis begleitet werden oder jederzeit auch andere KundInnen als Übersetzungshilfe herangezogen werden können. 


\section{KundInnen des AMS Jugendliche}

\subsection{Charakteristika der Jugendlichen mit Migrationshintergrund}

\subsubsection{Fakten zum Migrationshintergrund}

Mehr als ein Drittel (37\%) der Jugendlichen stammt aus einem der Nachfolgestaaten des ehemaligen Jugoslawiens, $32 \%$ aus der Türkei. Wie auch in der Wiener Bevölkerung stellen diese zwei Herkunftsgruppen den größten Anteil unter den migrantischen KundInnen der RGS Jugendliche dar.

Die anderen Herkunftsländer der Kundlnnen mit Migrationshintergrund spielen eine vergleichsweise geringere Rolle, wie aus Grafik 5 ersichtlich. Jeweils $9 \%$ kommen aus Osteuropa und Russland bzw. aus dem Nahen und Mittleren Osten. Rd. $5 \%$ der MigrantInnen haben Wurzeln in Asien und $4 \%$ in Afrika.

Grafik 5 Art des Migrationshintergrundes der Jugendlichen, Anteil der migrantischen Jugendlichen in Prozent

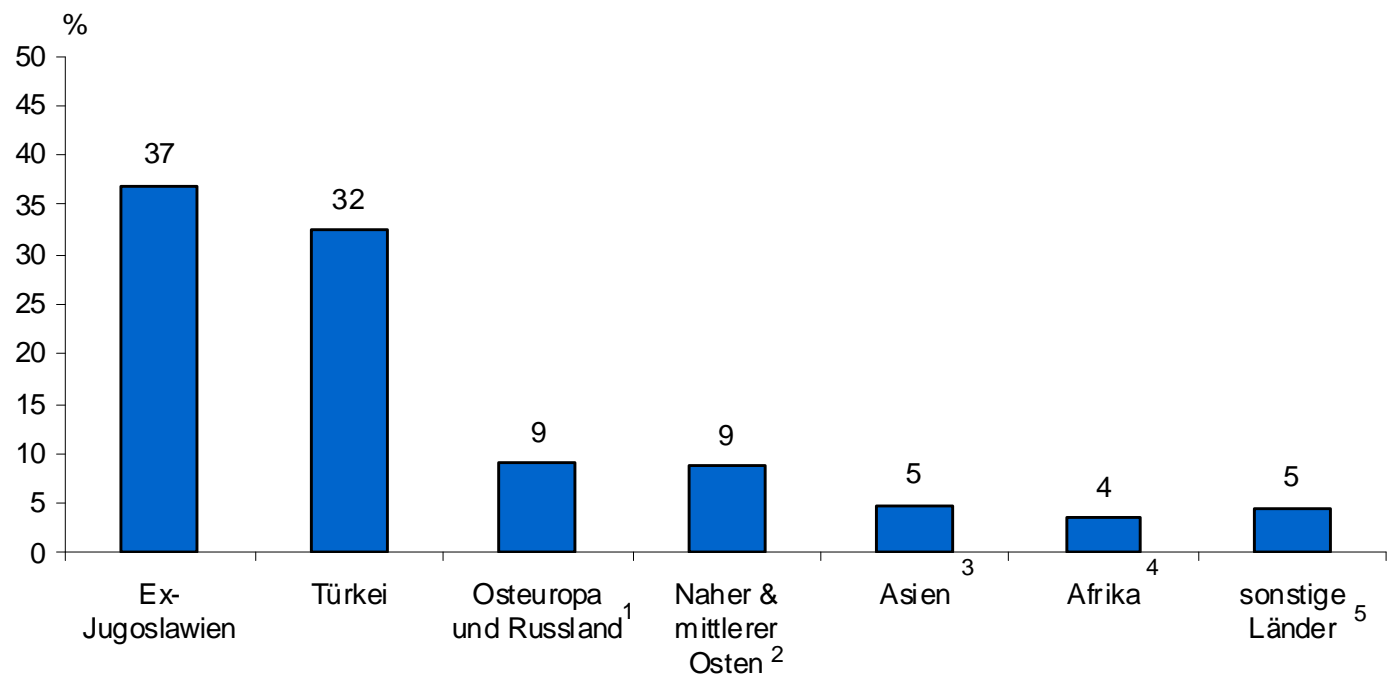

${ }^{1}$ Russland, Ukraine, Tschetschenien, Ungarn, Polen, Rumänien, Slowakei, Tschechische Republik

2 Ägypten, Iran, Irak, Afghanistan, Pakistan, Tadschikistan

${ }^{3}$ China, Indien, Philippinen, Sri Lanka, Thailand, Bangladesh

${ }^{4}$ DR Kongo, Ghana, Kenia, Nigeria, Äthiopien, Somalia, Tunesien

${ }^{5}$ Australien, Dominikanische Republik, Brasilien, Chile, Deutschland, Italien, Schweiz

Quelle: KMU FORSCHUNG AUSTRIA, Befragung der jugendlichen AMS-KundInnen, n=311

Rd. zwei Drittel (64 \%) der befragten jugendlichen MigrantInnen sind in Österreich geboren und leben bereits in zweiter Generation in Österreich (siehe Grafik 6). Während fast drei Viertel (73\%) der Jugendlichen mit Migrationshintergrund aus Ex-Jugoslawien und der Türkei in Österreich geboren sind, ist mehr als die Hälfte der Befragten (57\%) mit einem anderen Migrationshintergrund, insbesondere Jugendliche aus dem Nahen und Mittleren Osten (78\%), im Ausland auf die Welt gekommen (1. Generation). 
Grafik 6 Geburtsort der Jugendlichen, Anteil der migrantischen Jugendlichen nach Migrationshintergrund in Prozent

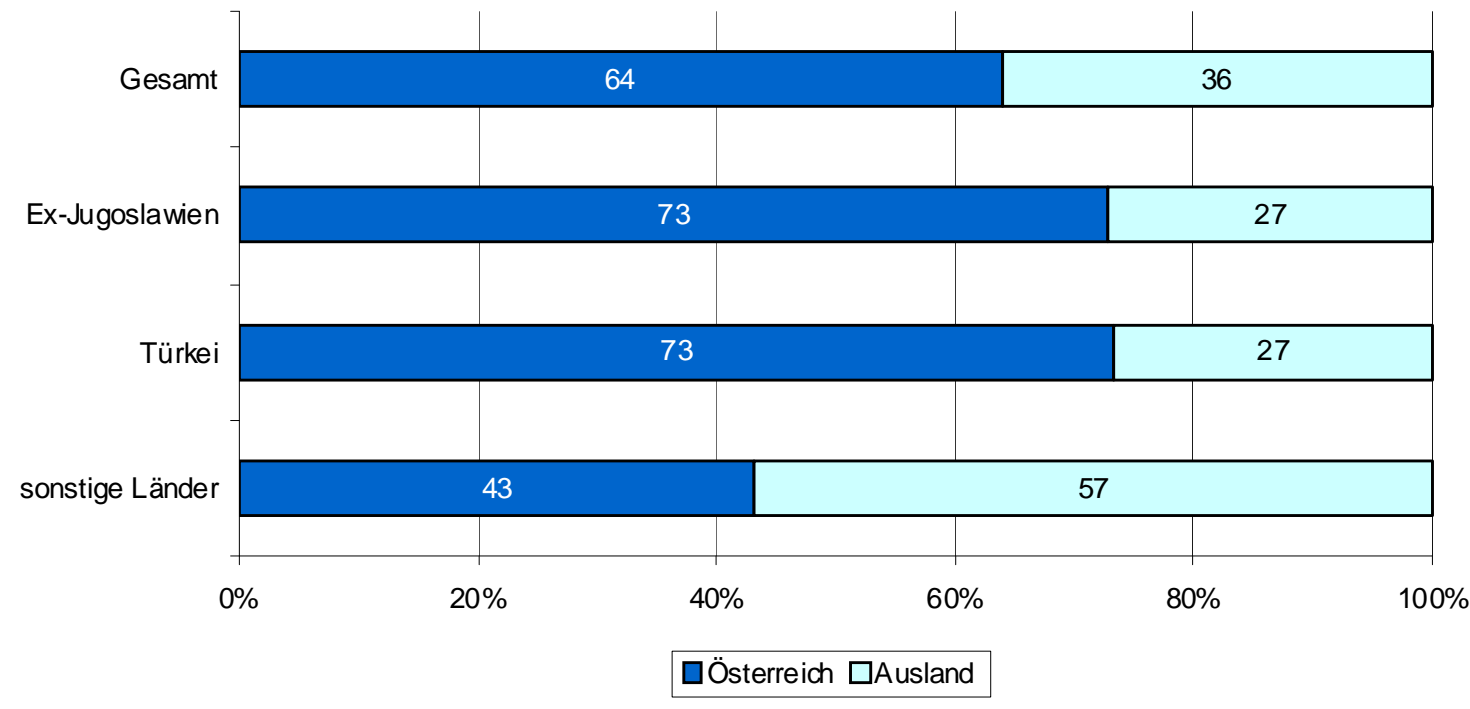

Quelle: KMU FORSCHUNG AUSTRIA, Befragung der jugendlichen AMS-KundInnen, $\mathrm{n}=310$

Der Großteil der Jugendlichen (48\%), die im Ausland geboren sind, und insbesondere jene aus der Türkei (67\%), kamen im Alter von 7 bis 15 Jahren nach Österreich, wie Grafik 7 zeigt. $52 \%$ der Jugendlichen, die in Ex-Jugoslawien geboren sind, kamen bereits vor ihrem 6. Lebensjahr nach Österreich im Vergleich zu $32 \%$ aller im Ausland Geborenen. Jugendliche aus anderen Ländern kamen überdurchschnittlich oft nach Österreich als sie bereits älter als 15 Jahre alt waren (37\% vs. $20 \%$ ), d. h. nach dem schulpflichtigen Alter.

Grafik 7 Alter zum Zeitpunkt der Immigration der im Ausland geborenen Jugendlichen, Anteil der migrantischen Jugendlichen nach Altersgruppen und Migrationshintergrund in Prozent

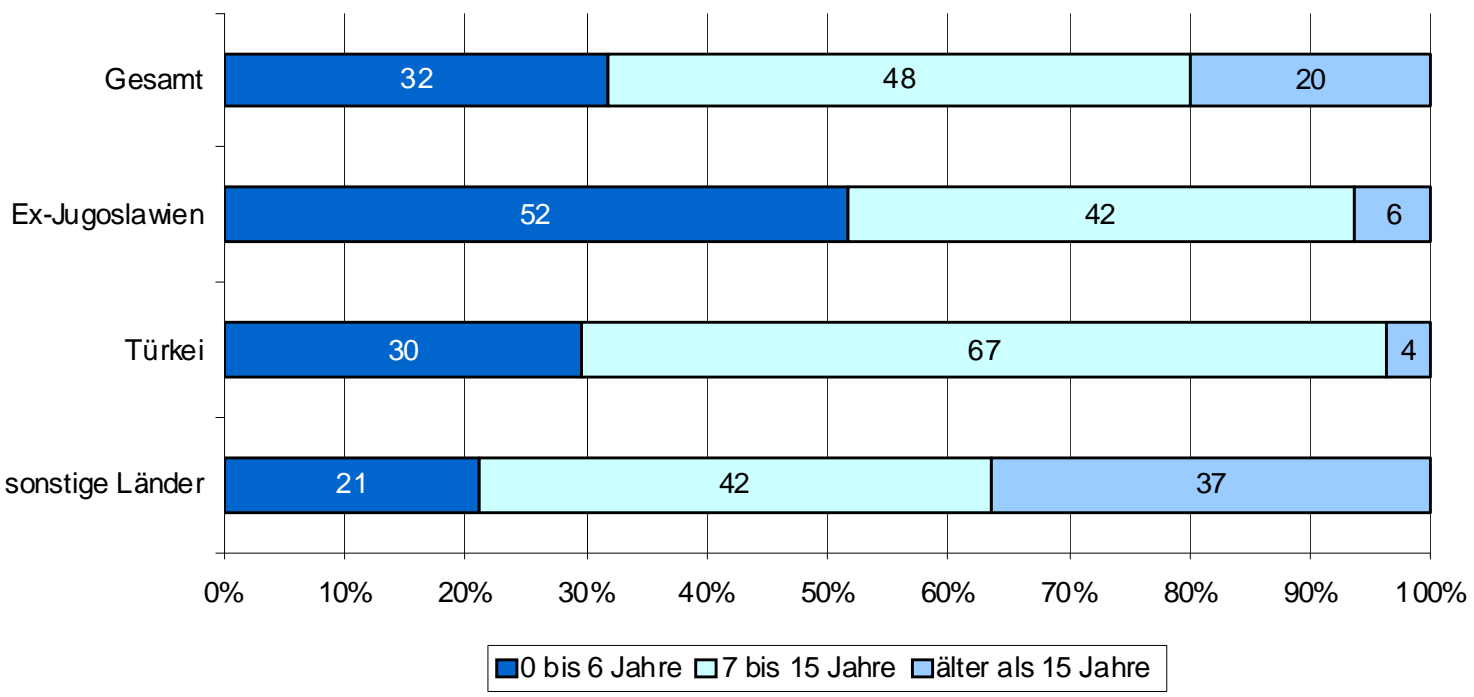

Quelle: KMU FORSCHUNG AUSTRIA, Befragung der jugendlichen AMS-KundInnen, n=110 
Insgesamt besitzen rd. zwei Drittel der befragten MigrantInnen die österreichische Staatsbürgerschaft (siehe Grafik 8). Unter den aus der Türkei stammenden Jugendlichen sind es sogar $82 \%$. Der Anteil der österreichischen StaatsbürgerInnen ist naturgemäß unter den MigrantInnen, die in Österreich geboren sind, deutlich höher (84\%) als unter den im Ausland geborenen Personen (37\%).

Grafik 8 Staatsbürgerschaft der Jugendlichen, Anteil der migrantischen Jugendlichen nach Migrationshintergrund in Prozent

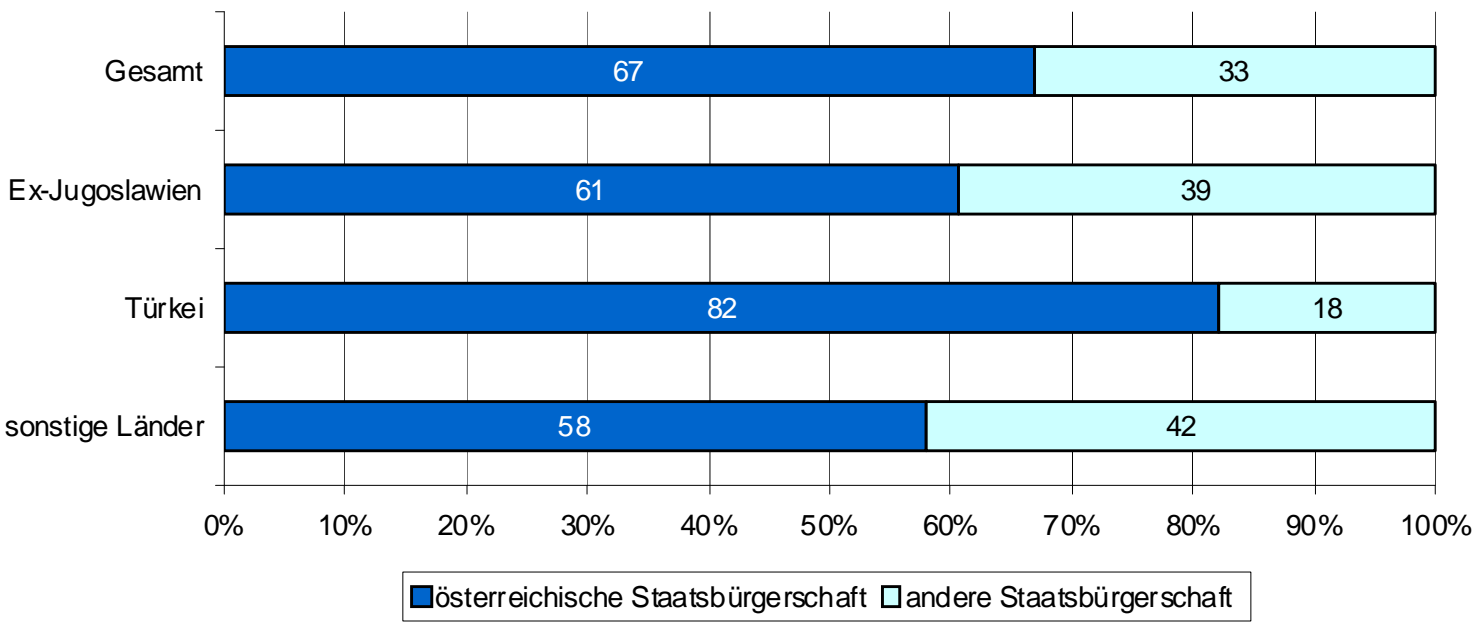

Quelle: KMU FORSCHUNG AUSTRIA, Befragung der jugendlichen AMS-KundInnen, $\mathrm{n}=310$

\subsubsection{Migration und Integration}

Die Beherrschung und der Gebrauch der deutschen Sprache sind wichtige Vorraussetzungen für die Integration in den österreichischen Arbeitsmarkt und stellen somit wichtige Integrationsfaktoren dar. Der Großteil der befragten MigrantInnen ist in Österreich geboren und hat auch hier die Schule besucht. Nichtsdestotrotz sind mangelnde oder fehlerhafte Deutschkenntnisse ein häufiges Problem bei den arbeitsuchenden MigrantInnen. Dies ist auf verschiedene Faktoren zurückzuführen wie z.B., dass mit dem Erwerb der deutschen Sprache zu spät begonnen wurde (z.B. erst in der Volksschule), die Muttersprache selbst nicht ausreichend beherrscht wird, was den Erwerb einer weiteren Sprache erschwert oder die Jugendlichen aus bildungsfernen Familien kommen. Anstatt eine Chance für die berufliche Zukunft darzustellen, wird die Mehrsprachigkeit in diesen Fällen zu einem Stolperstein für eine erfolgreiche Schullaufbahn und für eine Arbeitsmarktintegration der Jugendlichen.

$44 \%$ der befragten MigrantInnen gaben an, dass sie mit ihren Eltern ausschließlich in ihrer Muttersprache kommunizieren, wie Grafik 9 zeigt. Bei den Jugendlichen der ersten Generation liegt dieser Anteil bei $64 \%$. Ebenfalls ist dieser Anteil unter den aus der Türkei stammenden Jugendlichen überdurchschnittlich hoch (54\%). Bei $47 \%$ der Befragten wird im Familienkreis mehr als eine Sprache gesprochen. Deutsch ist hier in den häufigsten Fällen die Zweitsprache. Unter den Jugendlichen der zweiten Generation ist diese Konstellation häufiger vorzufinden (53\%), ebenso bei den Jugendlichen mit ex-jugoslawischem Hintergrund (57\%). Ausschließlich Deutsch sprechen $9 \%$ der Jugendlichen mit den Eltern, insbesondere die Jugendlichen der zweiten Generation (13\%) und MigrantInnen aus anderen Ländern (19 \%). 
Grafik 9 Gesprochene Sprache mit den Eltern, Anteil der migrantischen Jugendlichen nach Generation und Migrationshintergrund in Prozent

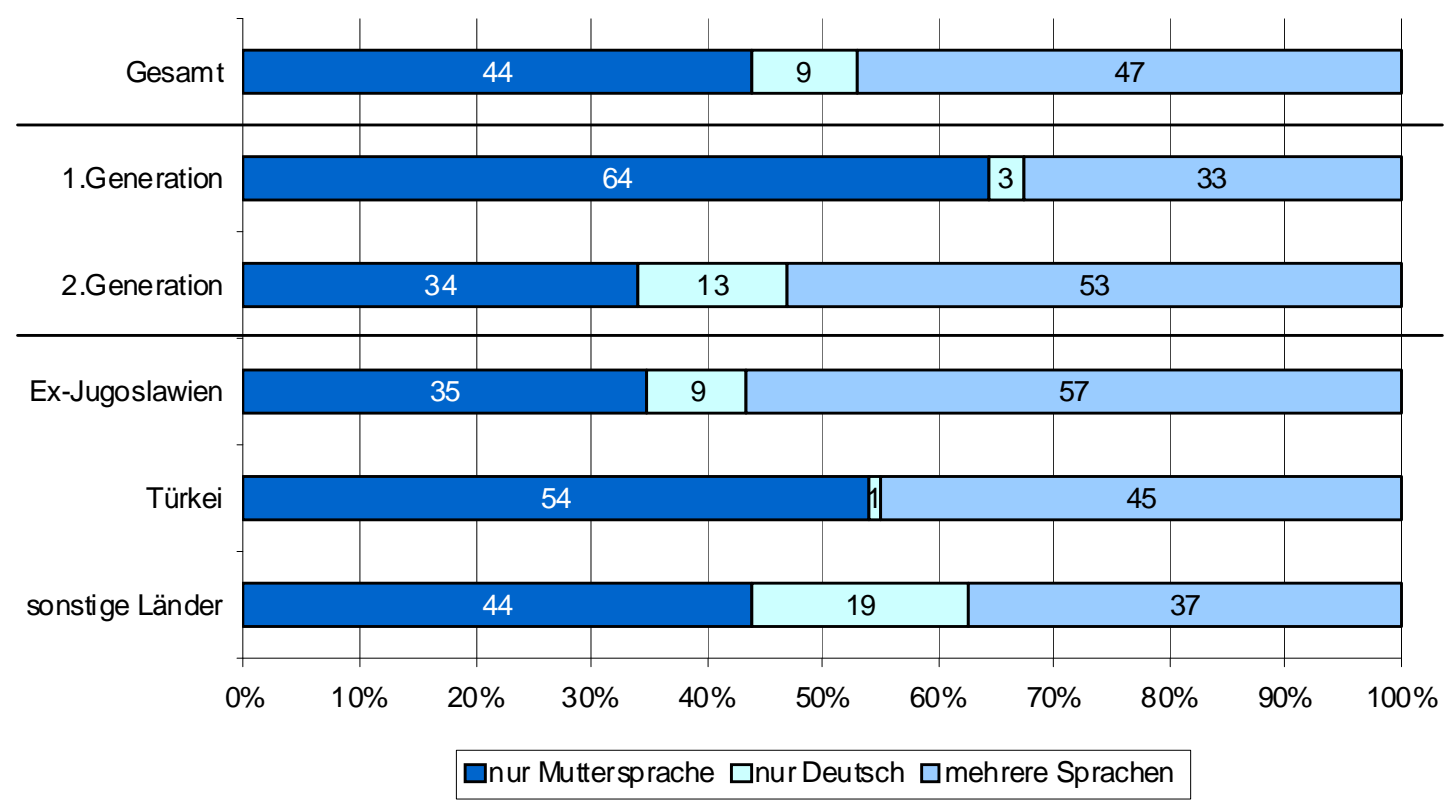

Quelle: KMU FORSCHUNG AUSTRIA, Befragung der jugendlichen AMS-KundInnen, $n=306$

Mit den Geschwistern spricht mehr als ein Drittel (36 \%) der Jugendlichen nur Deutsch, insbesondere die Jugendlichen der zweiten Generation (47\%), wie aus Grafik 10 ersichtlich. Am häufigsten werden mehrere Sprachen mit den Geschwistern gesprochen (45\%), ebenso bei den Jugendlichen aus Ex-Jugoslawien als auch bei jenen aus der Türkei. Ausschließlich in der Muttersprache unterhalten sich knapp ein Fünftel der befragten MigrantInnen (19\%) mit ihren Geschwistern, wobei dieser Anteil überdurchschnittlich hoch ist bei den Jugendlichen aus den anderen Ländern (28 \%).

Grafik 10 Gesprochene Sprache mit den Geschwistern, Anteil der migrantischen Jugendlichen nach Generation und Migrationshintergrund in Prozent

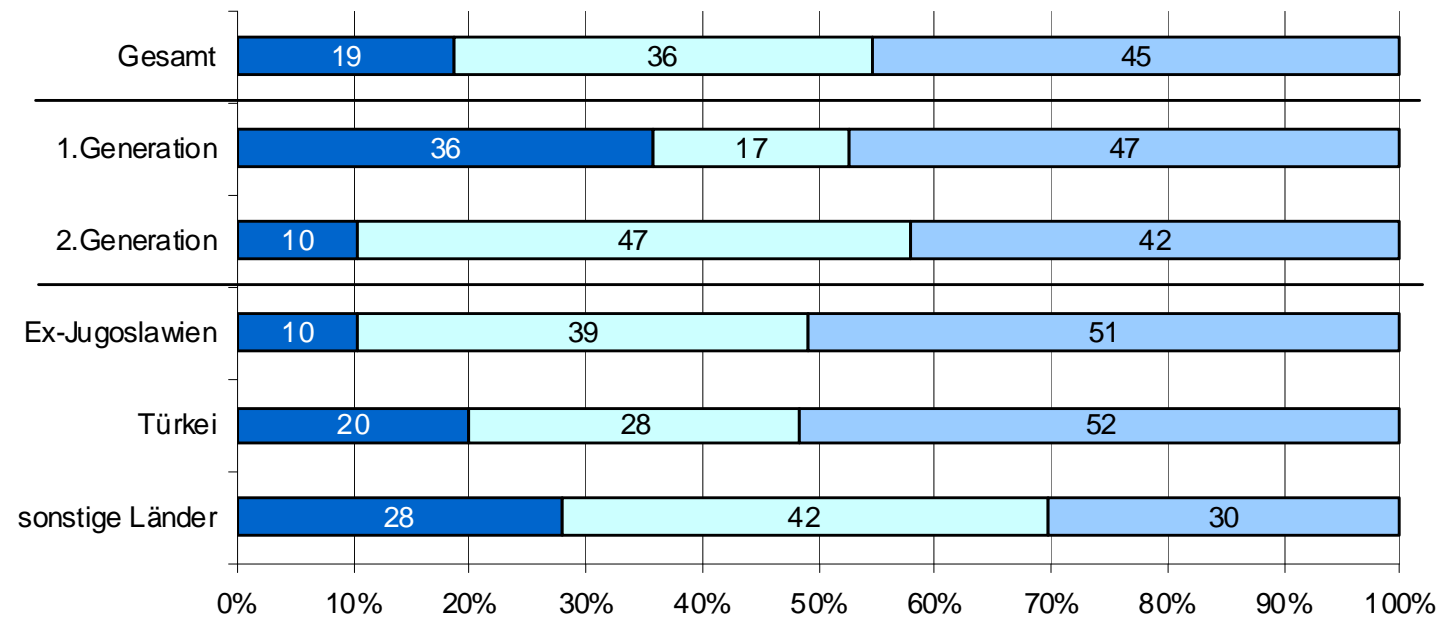

$\square$ nur Muttersprache $\square$ nur Deutsch $\square$ mehrere Sprachen

Quelle: KMU FORSCHUNG AUSTRIA, Befragung der jugendlichen AMS-KundInnen, $\mathrm{n}=\mathbf{2 8 0}$ 
Bei mehr als der Hälfte der befragten MigrantInnen (57\%) werden im Freundeskreis mehrere Sprachen gesprochen, insbesondere bei den Jugendlichen aus Ex-Jugoslawien (63\%) und der Türkei (67\%). Mehr als ein Drittel der MigrantInnen spricht ausschließlich Deutsch mit seinen FreundInnen. Dies ist am häufigsten bei den Jugendlichen der zweiten Generation und bei den Jugendlichen mit einem anderen Migrationshintergrund wie z.B. aus Asien, Osteuropa und Russland und Afrika der Fall (siehe Grafik 11). Dies kann darauf zurückgeführt werden, dass Jugendliche aus Ex-Jugoslawien und der Türkei in der Schule und im Freundeskreis eher auf Personen treffen, die ihre Muttersprache beherrschen.

Grafik 11 Gesprochene Sprache mit den Freunden, Anteil der migrantischen Jugendlichen nach Generation und Migrationshintergrund in Prozent

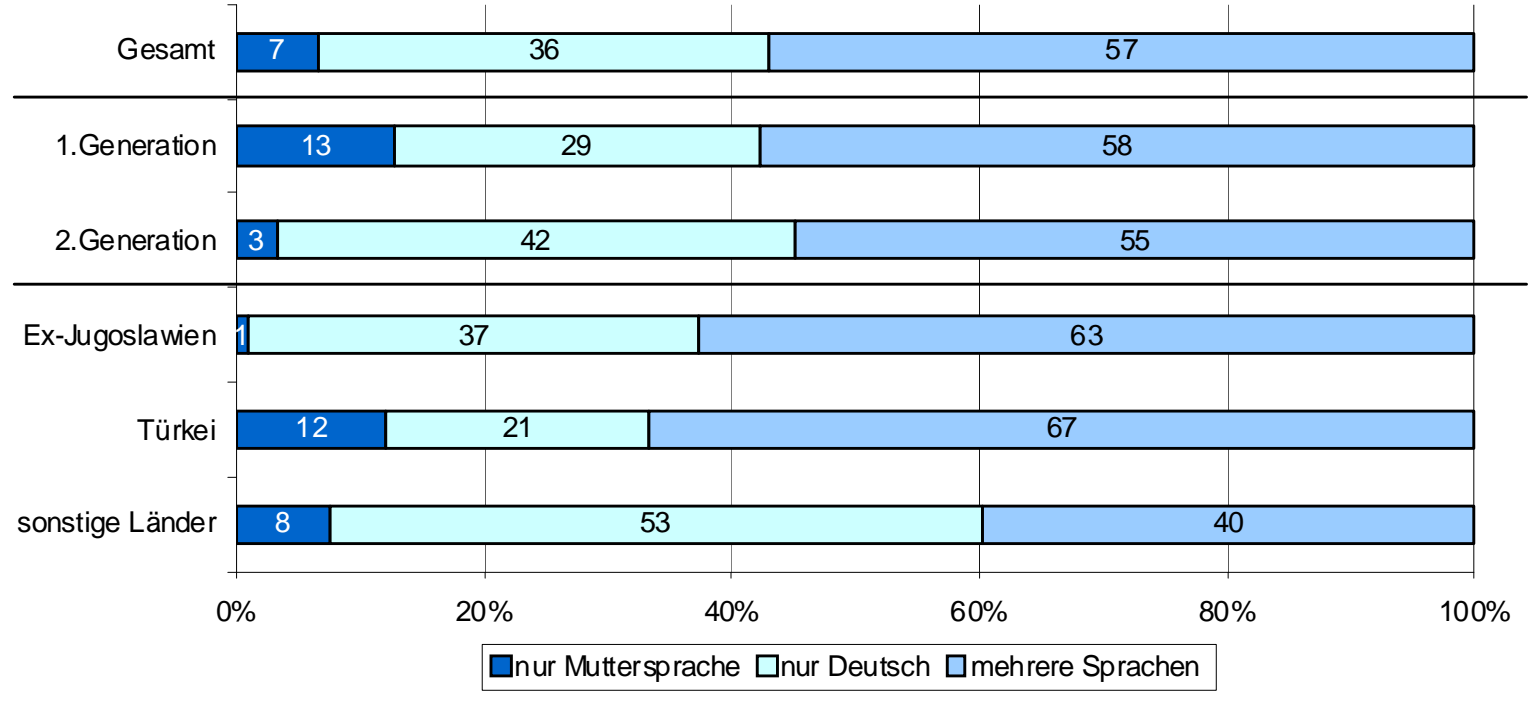

Quelle: KMU FORSCHUNG AUSTRIA, Befragung der jugendlichen AMS-KundInnen, n=307

Die jugendlichen MigrantInnen wurden ebenfalls zur Rolle der Traditionen des Herkunftslandes innerhalb ihrer Familien befragt und insbesondere über die Pflege der Feiertage, der Essgewohnheiten oder von etwaigen Bekleidungsvorschriften. Insgesamt gaben $89 \%$ der befragten MigrantInnen an, dass ihre Familie die Traditionen ihres Herkunftslandes pflegen, in erster Linie nannten diese Jugendlichen die Einhaltung der Feiertage (90\%), wie Grafik 12 zeigt. Weitere $89 \%$ gaben an, dass die Essgewohnheiten des Herkunftslandes in der Familie gepflegt werden, insbesondere in Familien aus der Türkei, dem Nahen und Mittleren Osten sowie aus Afrika. Bekleidungsvorschriften spielen in rd. einem Drittel der Familien eine Rolle (36\%), am häufigsten in Familien aus der Türkei (51\%) und dem Nahen bzw. Mittleren Osten (45\%), d. h. aus vom Islam geprägten Kulturkreisen, während z. B. nur $17 \%$ aus ExJugoslawien spezielle Bekleidungsvorschriften beachten. 
Grafik 12 Pflege der Traditionen des Herkunftslandes in Bezug auf die Feiertage, Anteil der migrantischen Jugendlichen nach Migrationshintergrund in Prozent

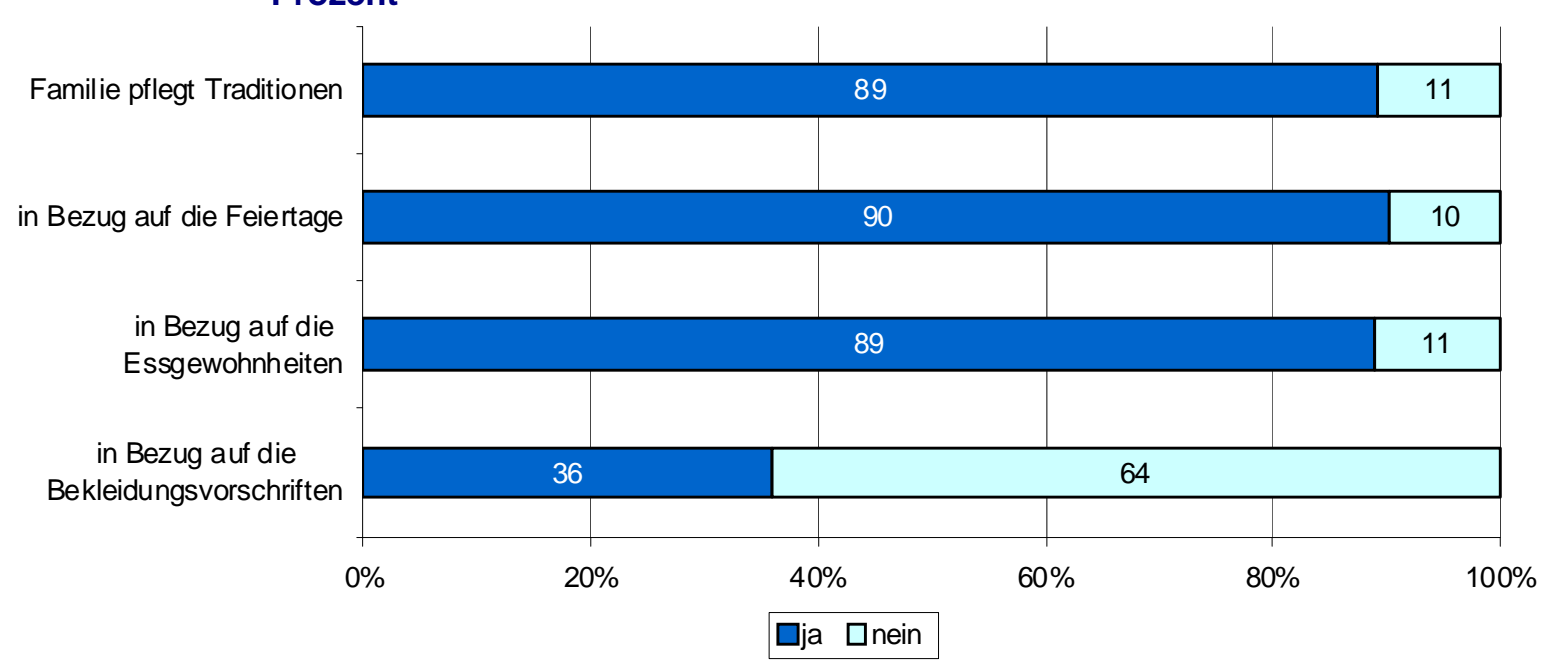

Quelle: KMU FORSCHUNG AUSTRIA, Befragung der jugendlichen AMS-KundInnen, $\mathrm{n}=240-278$

$45 \%$ der befragten MigrantInnen deklarierten, sich zu Österreich zugehörig zu fühlen, wobei dieser Anteil unter den Jugendlichen der zweiten Generation (53\%) höher ist als unter jenen der ersten (30\%) (siehe Grafik 13). Bei letzteren ist das Zugehörigkeitsgefühl zum Herkunftsland am stärksten ausgeprägt (49\%). Das Zugehörigkeitsgefühl zu Österreich ist unter den Jugendlichen aus der Türkei (48\%) als auch aus Ex-Jugoslawien (42 \%) ähnlich stark ausgeprägt.

\section{Grafik 13 Zugehörigkeitsgefühl der Jugendlichen, Anteil der migrantischen Jugend-} lichen nach Generation in Prozent

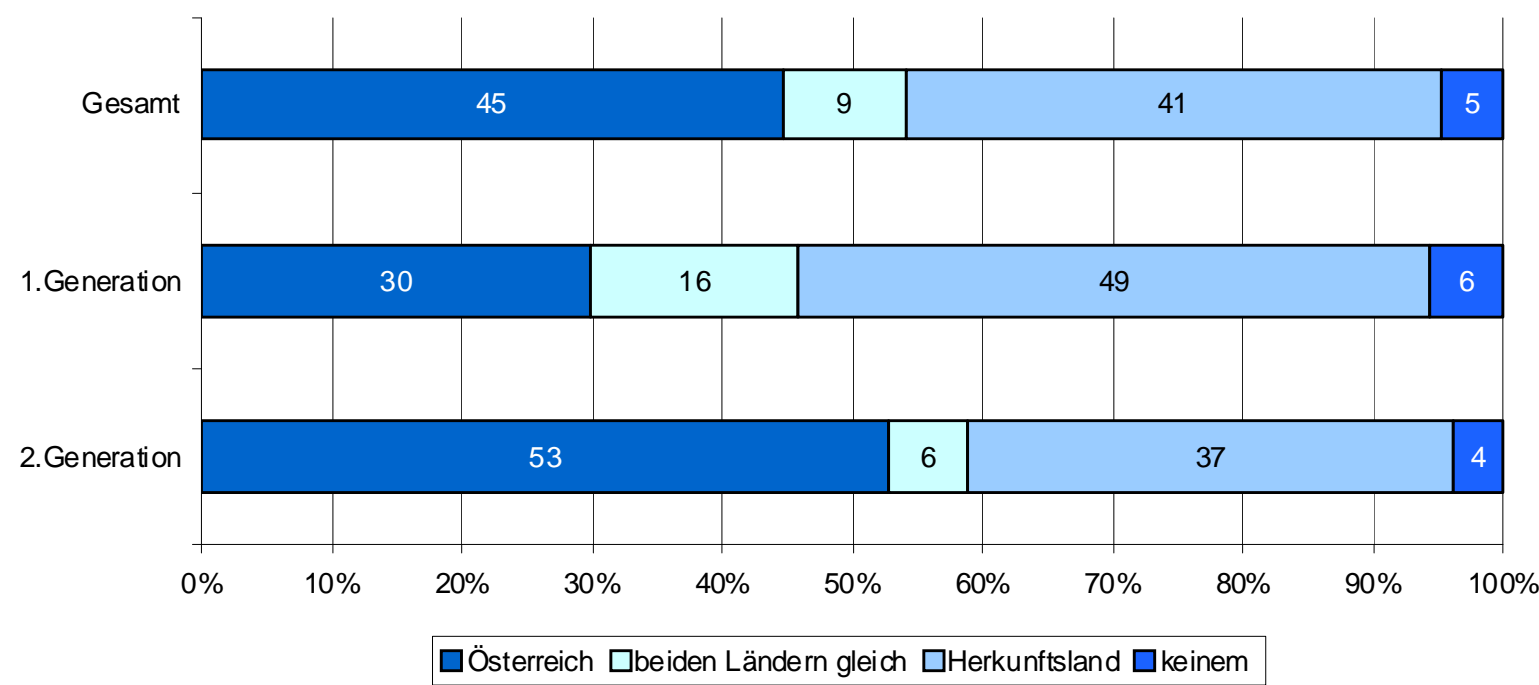

Quelle: KMU FORSCHUNG AUSTRIA, Befragung der jugendlichen AMS-Kundlnnen, $n=298$ 


\subsection{Beschäftigungssituation und Ausbildungshintergrund der Eltern}

Die Frage nach der Beschäftigungs- und Ausbildungssituation der Eltern der befragten Jugendlichen ergab - wie bereits in der Befragung von 2007 (vgl. Heckl et al., 2007) ein differenziertes Bild. Während die Väter, unabhängig vom Migrationshintergrund, in hohem Ausmaß erwerbstätig sind, ist dies bei den Müttern deutlich weniger der Fall, wobei starke Unterschiede je nach Migrationshintergrund vorliegen. In Bezug auf die Beschäftigungs- und Ausbildungssituation gibt es ebenfalls deutliche Unterschiede zwischen den Eltern mit und ohne Migrationshintergrund.

$80 \%$ der befragten Jugendlichen gaben an, dass ihre Väter in aufrechten Arbeitsverhältnissen stehen, wie Grafik 14 zeigt. Bei den Vätern der Jugendlichen ohne Migrationshintergrund waren es sogar $85 \%$. Mehr als ein Zehntel der Väter sind wie ihre Kinder ebenfalls auf Arbeitssuche. Rd. $8 \%$ hingegen sind nicht (mehr) erwerbstätig bzw. sind bereits in Pension.

Grafik 14 Erwerbsstatus der Väter der Jugendlichen, Anteil der befragten Jugendlichen nach Migrationshintergrund in Prozent

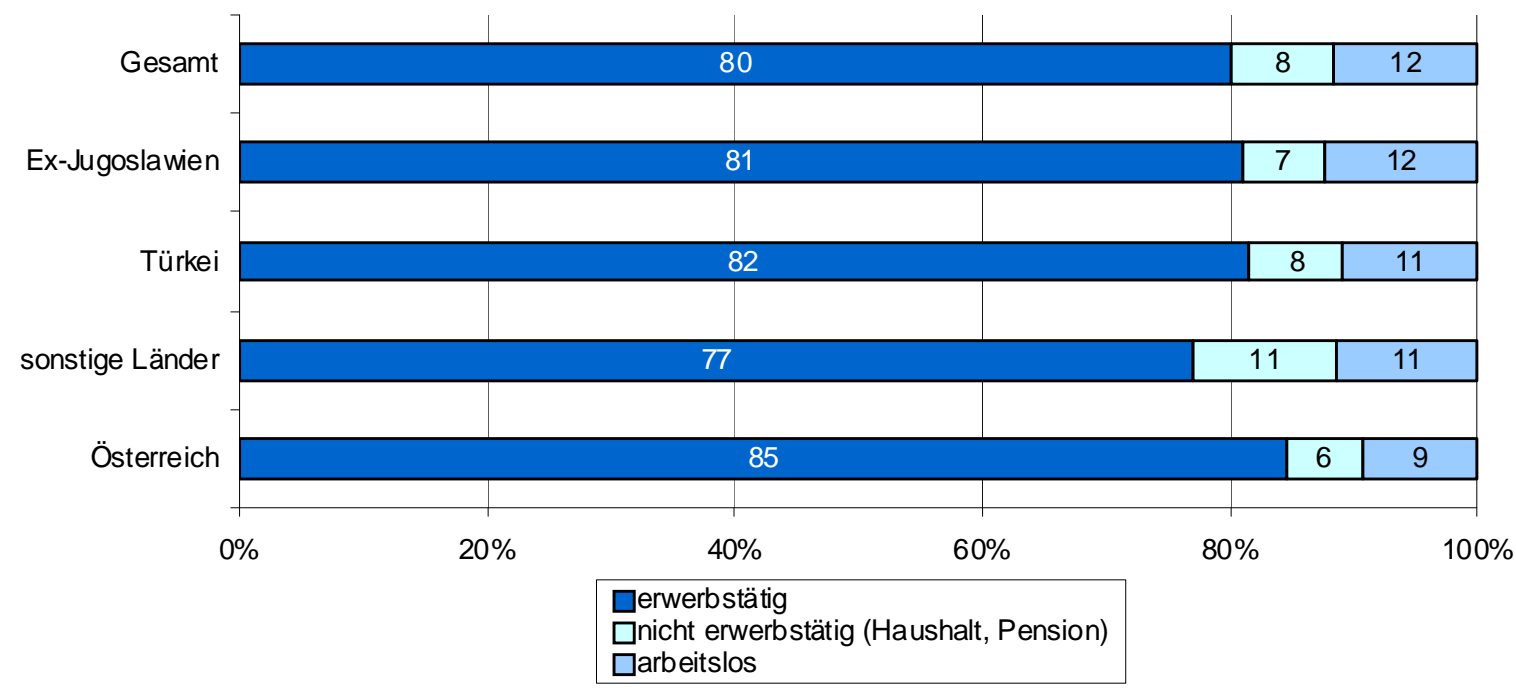

Quelle: KMU FORSCHUNG AUSTRIA, Befragung der jugendlichen AMS-KundInnen, n=364 
Der Großteil der Väter - und insbesondere jene mit Migrationshintergrund - arbeitet im Bauwesen und Baunebengewerbe, gefolgt vom Verkehr/Transport/Zustelldienste (siehe Tabelle 4). Die Väter der jugendlichen MigrantInnen arbeiten häufiger in niedrig qualifizierten Berufen und sind überdurchschnittlich oft in den Berufsbereichen Maschinen/Kfz/Metall und Hotel- und Gastgewerbe zu finden, während die Väter der österreichstämmigen Jugendlichen häufiger in den Branchen Elektro/Elektronik/Telekommunikation und Büro/Wirtschaft/Finanzwesen/Recht tätig sind.

Tabelle 4 Berufsbereiche der Väter der Jugendlichen, Anteil der befragten Jugendlichen nach Migrationshintergrund in Prozent

\begin{tabular}{|c|c|c|c|}
\hline & Gesamt & $\begin{array}{l}\text { ohne Migrations- } \\
\text { hintergrund }\end{array}$ & $\begin{array}{l}\text { mit Migrations- } \\
\text { hintergrund }\end{array}$ \\
\hline Bau, Baunebengewerbe und Holz & 25 & 20 & 27 \\
\hline Verkehr, Transport und Zustelldienste & 19 & 21 & 18 \\
\hline Maschinen, Kfz und Metall & 8 & 3 & 10 \\
\hline Hotel- und Gastgewerbe & 8 & 4 & 9 \\
\hline selbständige Tätigkeit & 7 & 7 & 7 \\
\hline Handel und Verkauf & 6 & 6 & 6 \\
\hline Reinigung und Hausbetreuung & 5 & 7 & 4 \\
\hline $\begin{array}{l}\text { Elektro, Elektronik und } \\
\text { Telekommunikation }\end{array}$ & 4 & 9 & 2 \\
\hline $\begin{array}{l}\text { Büro, Wirtschaft, Finanzwesen und } \\
\text { Recht }\end{array}$ & 3 & 7 & 1 \\
\hline $\begin{array}{l}\text { Landwirtschaft, Gartenbau und } \\
\text { Forstwirtschaft }\end{array}$ & 3 & 1 & 3 \\
\hline Soziales, Erziehung und Bildung & 3 & 1 & 3 \\
\hline Gesundheit und Medizin & 2 & 1 & 3 \\
\hline Sicherheitsdienste & 2 & 7 & 1 \\
\hline Sonstige Berufsbereiche & 7 & 4 & 8 \\
\hline Gesamt in Prozent & 100 & 100 & 100 \\
\hline
\end{tabular}


In Bezug auf die Schulausbildung gibt es ebenfalls Unterschiede zwischen den Vätern mit und ohne Migrationshintergrund. Ein Drittel der Väter mit Migrationshintergrund hat nur die Pflichtschule absolviert und rd. $8 \%$ lediglich die Volksschule (v. a. Väter aus der Türkei), wie aus Grafik 15 ersichtlich. $46 \%$ der Väter ohne Migrationshintergrund verfügen hingegen über einen Lehrabschluss und sind somit höher qualifiziert als die migrantischen Väter $(21 \%)$. Bei den Hochschulabschlüssen hingegen ist der Anteil unter den Migranten (6 \%) höher als unter den Österreichern (4\%). Mehr als ein Viertel der befragten Jugendlichen wusste über das Ausbildungsniveau ihrer Väter jedoch nicht Bescheid.

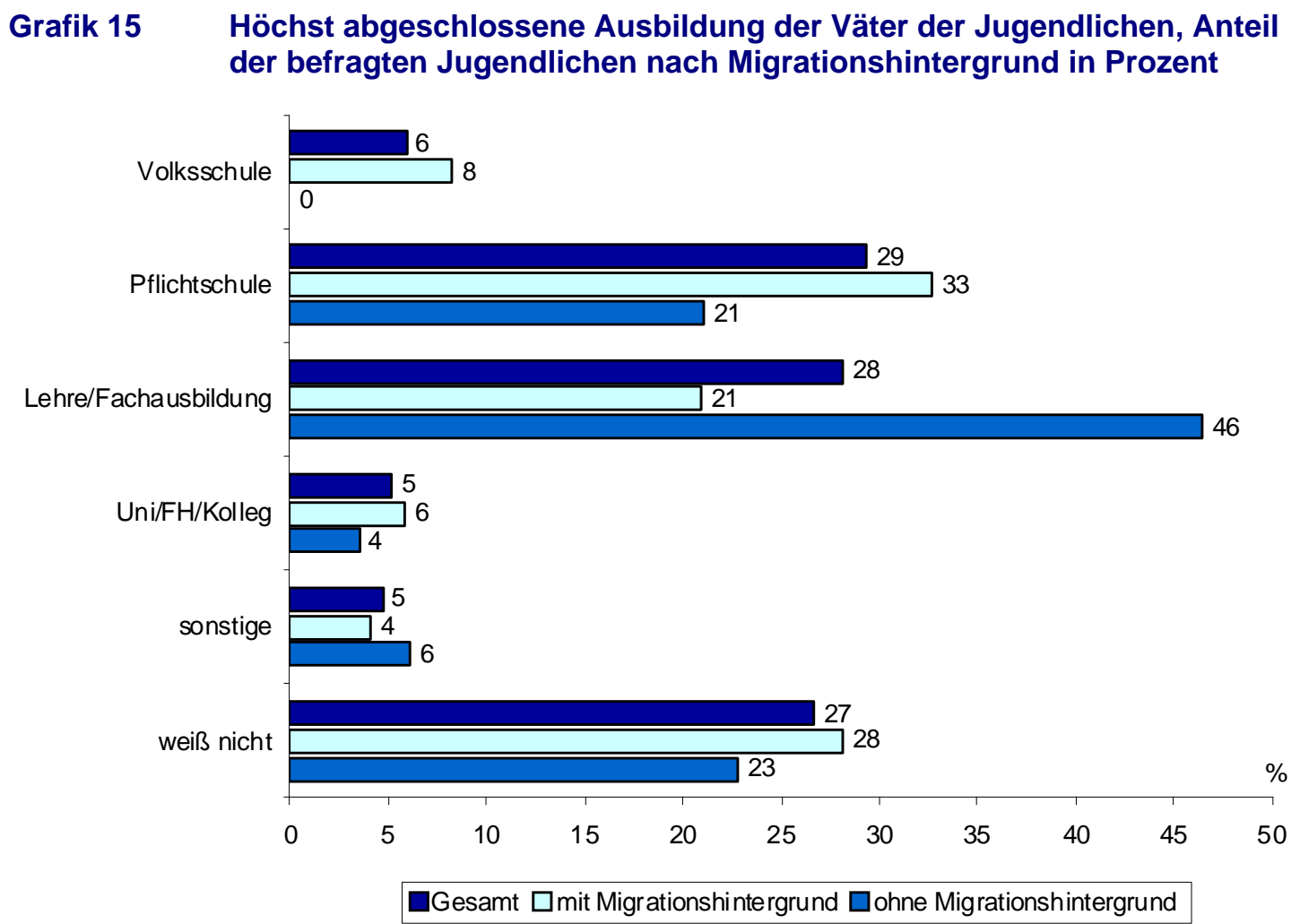

Quelle: KMU FORSCHUNG AUSTRIA, Befragung der jugendlichen AMS-KundInnen, n=405

In Bezug auf die Erwerbstätigkeit der Mütter gibt es deutliche Unterschiede je nach Herkunftsgruppe. Insgesamt ist mehr als die Hälfte der Mütter erwerbstätig (55 \%), insbesondere aber die österreichstämmigen Mütter (81 \%) und jene aus Ex-Jugoslawien (69\%), wie Grafik 16 zeigt. Die türkischen Mütter sind hingegen mehrheitlich (64\%) nicht erwerbstätig bzw. im Haushalt tätig - lediglich ein Drittel ist am Arbeitsmarkt integriert. Arbeitslos sind $5 \%$ der Mütter laut Auskunft ihrer Kinder. 
Grafik 16 Erwerbsstatus der Mütter der Jugendlichen, Anteil der befragten Jugendlichen nach Migrationshintergrund in Prozent

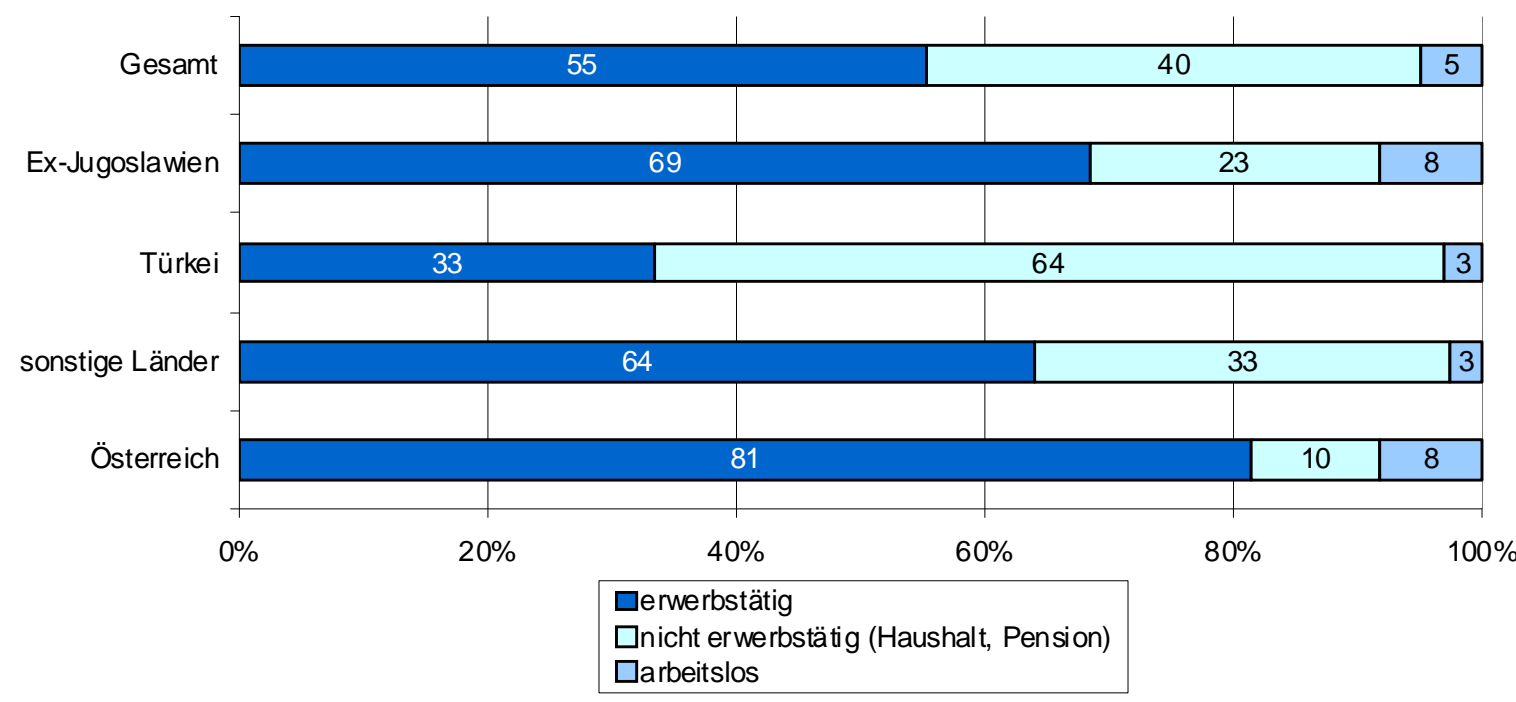

Quelle: KMU FORSCHUNG AUSTRIA, Befragung der jugendlichen AMS-KundInnen, n=390

In Hinblick auf die Berufsbereiche ist der Unterschied zwischen den Müttern der jugendlichen MigrantInnen und denen der österreichstämmigen Jugendlichen deutlich stärker ausgeprägt als bei den Vätern. Während die Migrantinnen am häufigsten in der Reinigung/Haubetreuung, im Hotel- und Gastgewerbe und der Gesundheit/Medizin arbeiten, sind die österreichstämmigen Mütter in erster Linie im Büro/Wirtschaft und im Handel/Verkauf tätig (siehe Tabelle 5).

Tabelle 5 Berufsbereiche der Mütter der Jugendlichen, Anteil der befragten Jugendlichen nach Migrationshintergrund in Prozent

\begin{tabular}{l|c|c|c}
\hline & Gesamt & $\begin{array}{c}\text { ohne Migrations- } \\
\text { hintergrund }\end{array}$ & $\begin{array}{c}\text { mit Migrations- } \\
\text { hintergrund }\end{array}$ \\
\hline Reinigung und Hausbetreuung & 25 & 7 & 35 \\
\hline Büro, Wirtschaft, Finanzwesen und Recht & 17 & 29 & 10 \\
\hline Hotel- und Gastgewerbe & 14 & 11 & 16 \\
\hline Gesundheit und Medizin & 11 & 9 & 13 \\
\hline Handel und Verkauf & 11 & 13 & 10 \\
\hline Soziales, Erziehung und Bildung & 8 & 9 & 7 \\
\hline selbständige Tätigkeit & 5 & 4 & 5 \\
\hline Verkehr, Transport und Zustelldienste & 2 & 5 & 5 \\
\hline Sonstige Berufsbereiche & 8 & 12 & 100 \\
\hline \hline Gesamt in Prozent & 100 & 100 & \\
\hline Quelle: KMU FORSCHUNG AUSTRIA, Befragung der jugendlichen AMS-Kundlnnen, $\mathrm{n}=210$ \\
\hline
\end{tabular}


In Bezug auf die Ausbildung gibt es ebenfalls große Unterschiede. Die migrantischen Mütter sind grundsätzlich weniger gebildet als die österreichstämmigen Mütter: Ebenso wie die Väter verfügt fast die Hälfte der österreichstämmigen Mütter über einen Lehrabschluss im Gegensatz zu nur $14 \%$ der migrantischen Mütter, wie Grafik 17 zeigt. Der Großteil der Migrantinnen (40\%) hat nur die Pflichtschule absolviert, $9 \%$ nur die Volksschule (v. a. Mütter aus der Türkei). Hingegen gibt es wie bei den Vätern mehr Mütter mit Hochschulabschluss unter den Migrantinnen (7\%) als unter den Österreicherinnen (4\%).

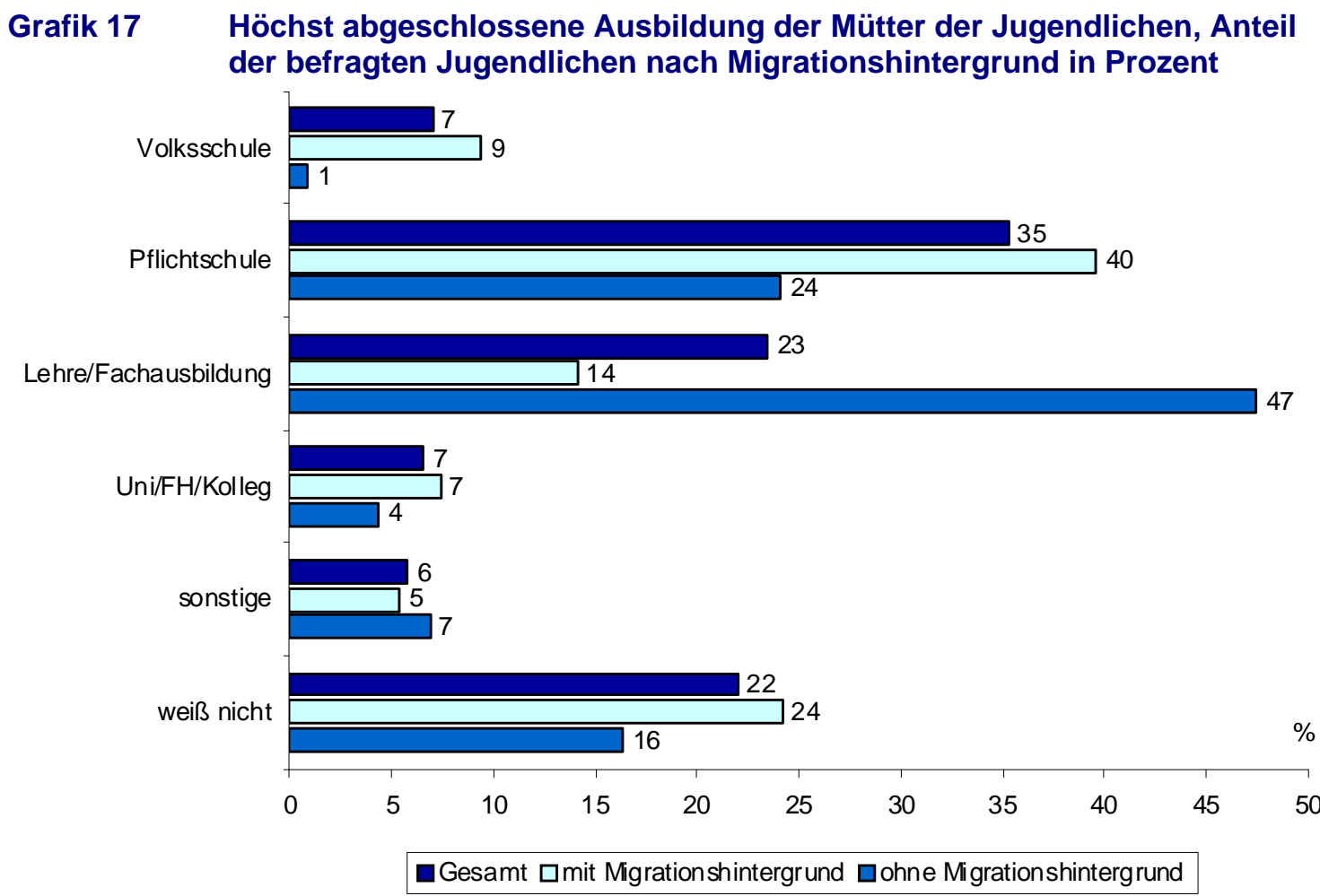

Quelle: KMU FORSCHUNG AUSTRIA, Befragung der jugendlichen AMS-KundInnen, n=414

Zusammenfassend lässt sich sagen, dass das Ausbildungsniveau der migrantischen Eltern und insbesondere der Mütter niedriger ist als das der österreichstämmigen. Ebenfalls sind die Eltern der befragten MigrantInnen viel häufiger in Sektoren mit einem hohen Anteil an niedrig qualifizierten Jobs tätig als die Eltern der österreichischen Jugendlichen. 


\section{Ausbildungshintergrund und Integration am Arbeitsmarkt}

\subsection{Schulkarriere}

Eine Grundausbildung sowie entsprechende Schulabschlüsse sind wichtige Faktoren, um die Integration von Jugendlichen am Arbeitsmarkt zu erleichtern. Ein wesentliches Problem der Jugendlichen, die beim AMS gemeldet sind, ist ihr niedriges Ausbildungsniveau. Hier zeigt sich, dass die niedrige Ausbildung der Eltern an die Kinder „weitervererbt" wird. Die Befragung ergab, dass rd. $43 \%$ der befragten Jugendlichen zuletzt die Hauptschule bzw. eine kooperative Mittelschule besucht haben (siehe Grafik 18). Im Polytechnischen Lehrgang waren knapp ein Viertel (23\%) der Befragten. Jeweils ein Zehntel der Jugendlichen hat eine berufsbildende mittlere oder höhere Schule besucht und lediglich $9 \%$ die AHS. Insgesamt haben $67 \%$ der befragten Jugendlichen unabhängig von Migrationshintergrund - die zuletzt besuchte Schule positiv abgeschlossen. Vor allem Hauptschule und Polytechnikum werden positiv absolviert, während der Besuch von mittleren oder höheren Schulen häufig nicht zum gewünschten Abschluss führt, wie auch ein Jugendlicher aus einer Fokusgruppe berichtet: „Ich habe die Handelsschule nach einem Jahr abgebrochen, die Noten waren nicht gut, dann war ich eine zeitlang daheim. "Insgesamt weisen jedoch $85 \%$ der Jugendlichen einen positiven und $12 \%$ einen negativen Pflichtschulabschluss auf. Nur $3 \%$ der Befragten haben die Matura. Bezüglich der Schulkarriere bestehen kaum Unterschiede zwischen Jugendlichen mit und ohne Migrationshintergrund.

Grafik 18 Zuletzt besuchte Schule der Jugendlichen, Anteil der befragten Jugendlichen nach Migrationshintergrund in Prozent

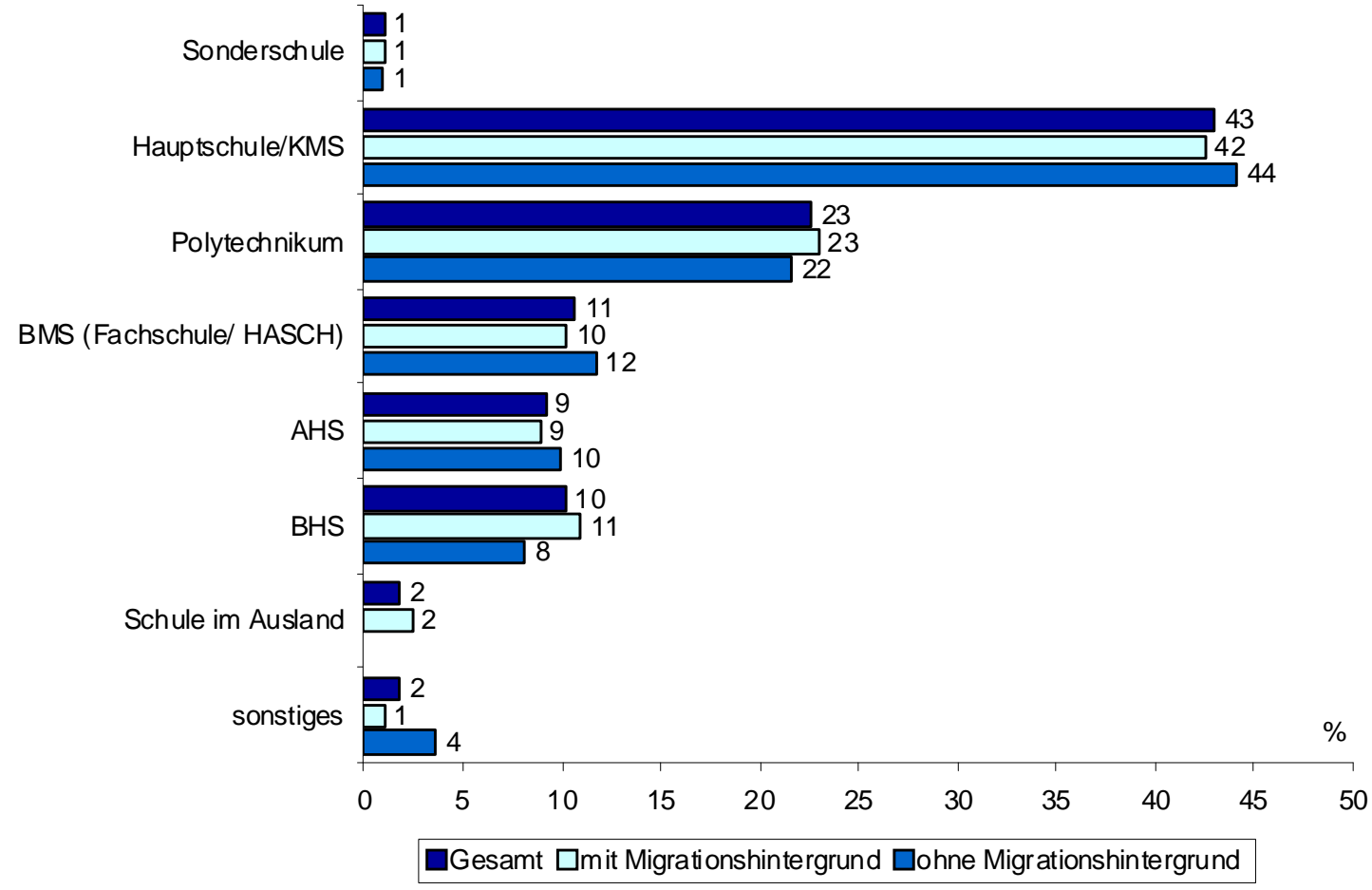

Quelle: KMU FORSCHUNG AUSTRIA, Befragung der jugendlichen AMS-KundInnen, n=403 
Rd. ein Drittel der befragten Jugendlichen (36\%) wurde während ihrer Schulzeit beim Lernen unterstützt. Am häufigsten hatten sie Hilfe von Familienmitgliedern, insbesondere die Jugendlichen aus österreichstämmigen Familien (64\% vs. $43 \%$ der migrantischen Jugendlichen), wie aus Grafik 19 ersichtlich. Dies ist wohl darauf zurückzuführen, dass die Eltern der migrantischen Jugendlichen nicht genügend Deutschkenntnisse haben, um ihren Kindern beim Lernen zu helfen. Von diesem Problem berichten auch die Jugendlichen bei den Fokusgruppen: „Die Familie unterstützt mich schon, aber die können nicht so gut Deutsch, daher mach ich alles selber." Die Jugendlichen mit Migrationshintergrund wurden häufiger als die österreichstämmigen Jugendlichen von den Lehrerlnnen in der Schule (26\% vs. $21 \%)$ und insbesondere von NachhilfelehrerInnen (24\% vs. $10 \%)$ unterstützt.

Grafik 19 Unterstützung der Jugendlichen beim Lernen während der Schulzeit, Anteil der befragten Jugendlichen nach Migrationshintergrund in Prozent

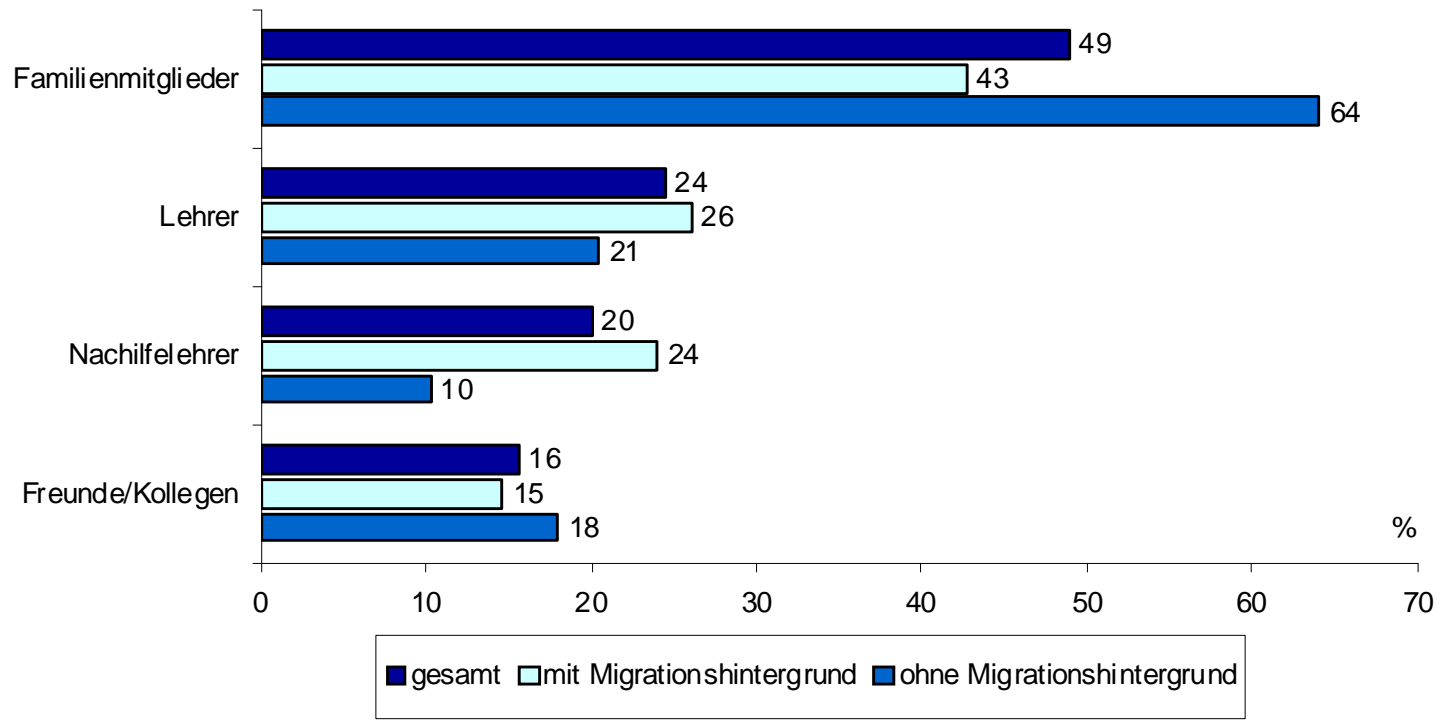

Quelle: KMU FORSCHUNG AUSTRIA, Befragung der jugendlichen AMS-KundInnen, n=135, Mehrfachnennungen möglich

Mehr als die Hälfte der befragten Jugendlichen (51\%) gab an, dass sie mehr Unterstützung gebraucht hätte, um einen besseren Abschluss zu erreichen (14\% viel mehr und $37 \%$ etwas mehr Hilfe). Insbesondere $19 \%$ der Mädchen (vs. $10 \%$ bei den Burschen) und $16 \%$ der MigrantInnen (vs. $8 \%$ der Österreichstämmigen) meinten, dass sie viel mehr Unterstützung benötigt hätten. Die Hilfe hätten sich die Jugendlichen in erster Linie von LehrerInnen und Familienmitgliedern gewünscht.

Die Lernumgebung zuhause ist ebenfalls für einen Schulerfolg wichtig. $70 \%$ der befragten Jugendlichen gaben an, dass sie immer zuhause ungestört lernen konnten, $18 \%$ nur manchmal und $12 \%$ selten oder nie (siehe Grafik 20). Die Mädchen als auch die MigrantInnen konnten weniger häufig in Ruhe zuhause lernen. 
Grafik 20 Möglichkeit eines ungestörten Lernens der Jugendlichen zuhause, Anteil der befragten Jugendlichen nach Migrationshintergrund und Geschlecht in Prozent

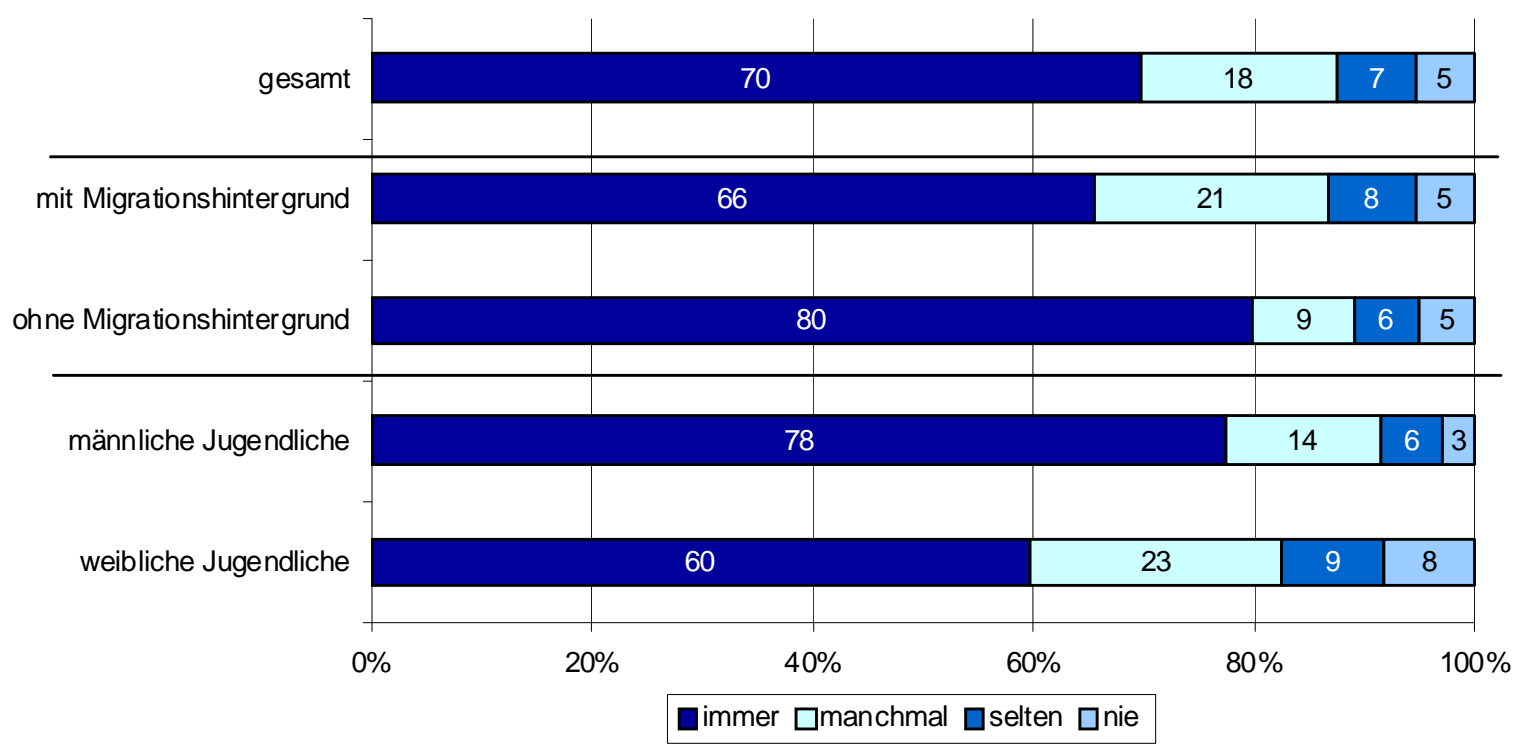

Quelle: KMU FORSCHUNG AUSTRIA, Befragung der jugendlichen AMS-KundInnen, n=420

Rd. ein Drittel der Eltern standen zudem im regelmäßigen Kontakt mit der Schule. $41 \%$ taten es nur fallweise, wenn es notwendig war und mehr als ein Viertel (27\%) hatte während der Schulzeit ihrer Kinder nie Kontakt mit der Schule, wie Grafik 21 zeigt. Tendenziell standen die österreichstämmigen Eltern viel häufiger in Kontakt mit der Schule bzw. den LehrerInnen als die Eltern mit Migrationshintergrund. Dies kann wieder auf die mangelnden Deutschkenntnisse der migrantischen Eltern zurückzuführen sein.

\section{Grafik 21 Häufigkeit der Kontakte der Eltern mit der Schule, Anteil der befragten Jugendlichen nach Migrationshintergrund in Prozent}

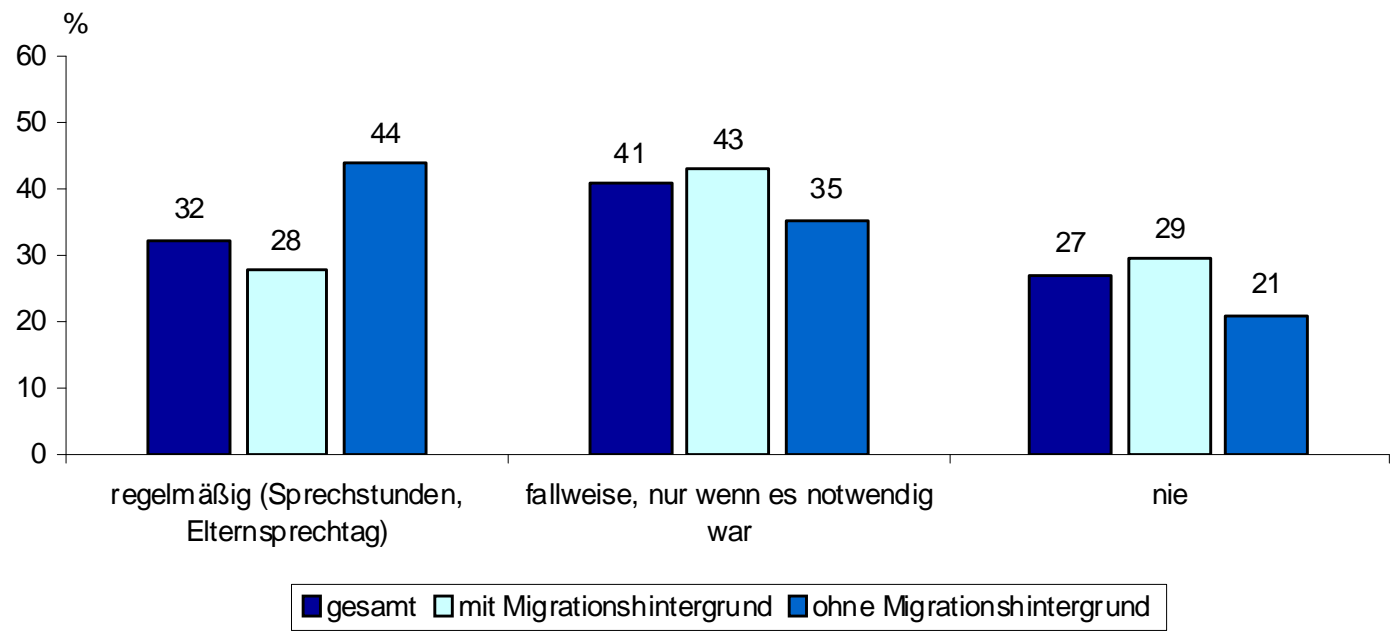

Quelle: KMU FORSCHUNG AUSTRIA, Befragung der jugendlichen AMS-KundInnen, n=415 
Vor diesem Hintergrund ist es auch nicht erstaunlich, dass nur $40 \%$ der migrantischen Eltern über das österreichische Schul- und Ausbildungssystem gut informiert sind im Vergleich zu $58 \%$ der österreichstämmigen Eltern (siehe Grafik 22). Mehr als ein Drittel der migrantischen Eltern kennen sich lediglich ein wenig und rd. ein Viertel überhaupt nicht aus (vs. 26 \% bzw. $15 \%$ der österreichstämmigen Eltern).

Grafik 22 Überblick der Eltern über das Schul- und Ausbildungssystem in Österreich, Anteil der befragten Jugendlichen nach Migrationshintergrund in Prozent

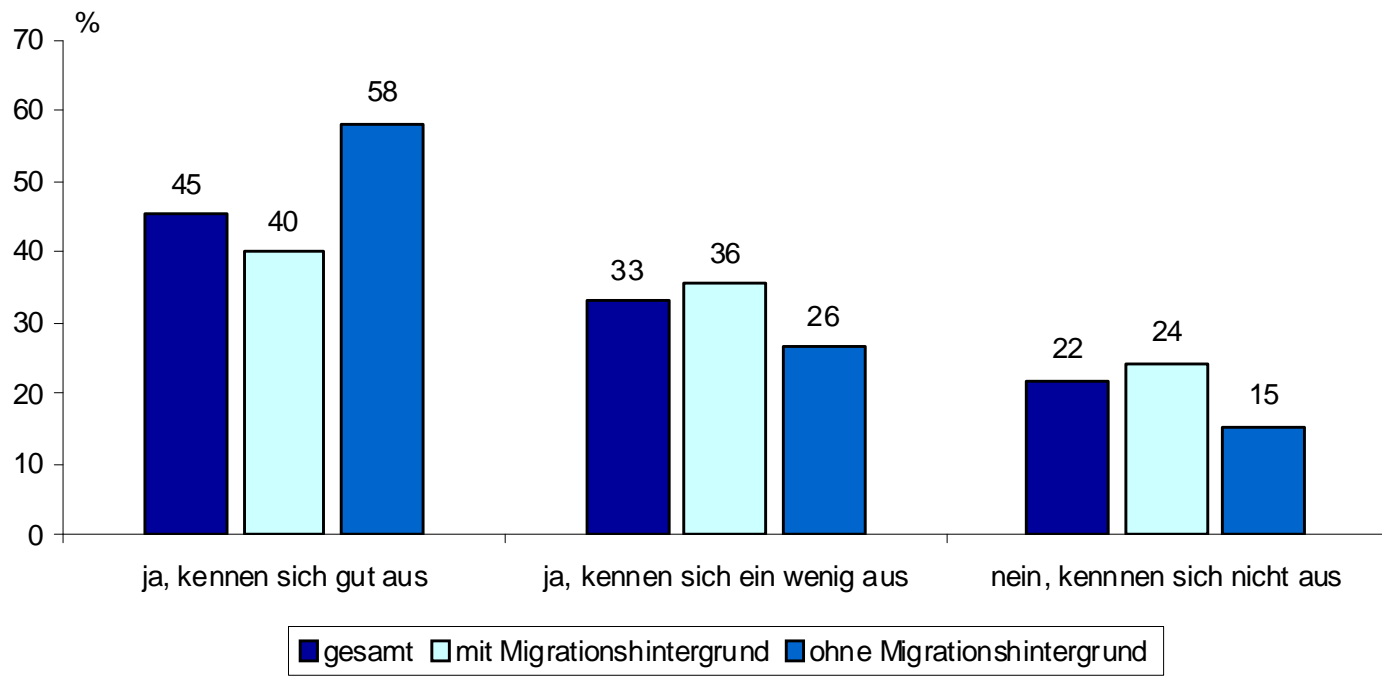

Quelle: KMU FORSCHUNG AUSTRIA, Befragung der jugendlichen AMS-Kundlnnen, $n=409$

Die Ergebnisse zeigen, dass die befragten Jugendlichen bereits während der Schulzeit mehr Unterstützung benötigt hätten, um zu einem besseren Schulabschluss zu kommen und ihre Chancen am Arbeitsmarkt zu steigern. Die mangelnde Unterstützung und auch der fehlende regelmäßige Kontakt zwischen den Eltern der Jugendlichen und der Schule, insbesondere bei migrantischen Eltern, erschwerten dies. 


\subsection{Erfahrungen am Arbeitsmarkt}

Trotz ihres jungen Alters haben insgesamt $79 \%$ der befragten Jugendlichen bereits Arbeitserfahrungen sammeln können, wie Grafik 23 zeigt. Die österreichstämmigen und die männlichen Jugendlichen jedoch häufiger (86\% und $83 \%$ ) als die migrantischen und die weiblichen Jugendlichen (76\% und $74 \%$ ).

Grafik 23 Berufliche Erfahrungen der Jugendlichen, Anteil der befragten Jugendlichen nach Migrationshintergrund und Geschlecht in Prozent

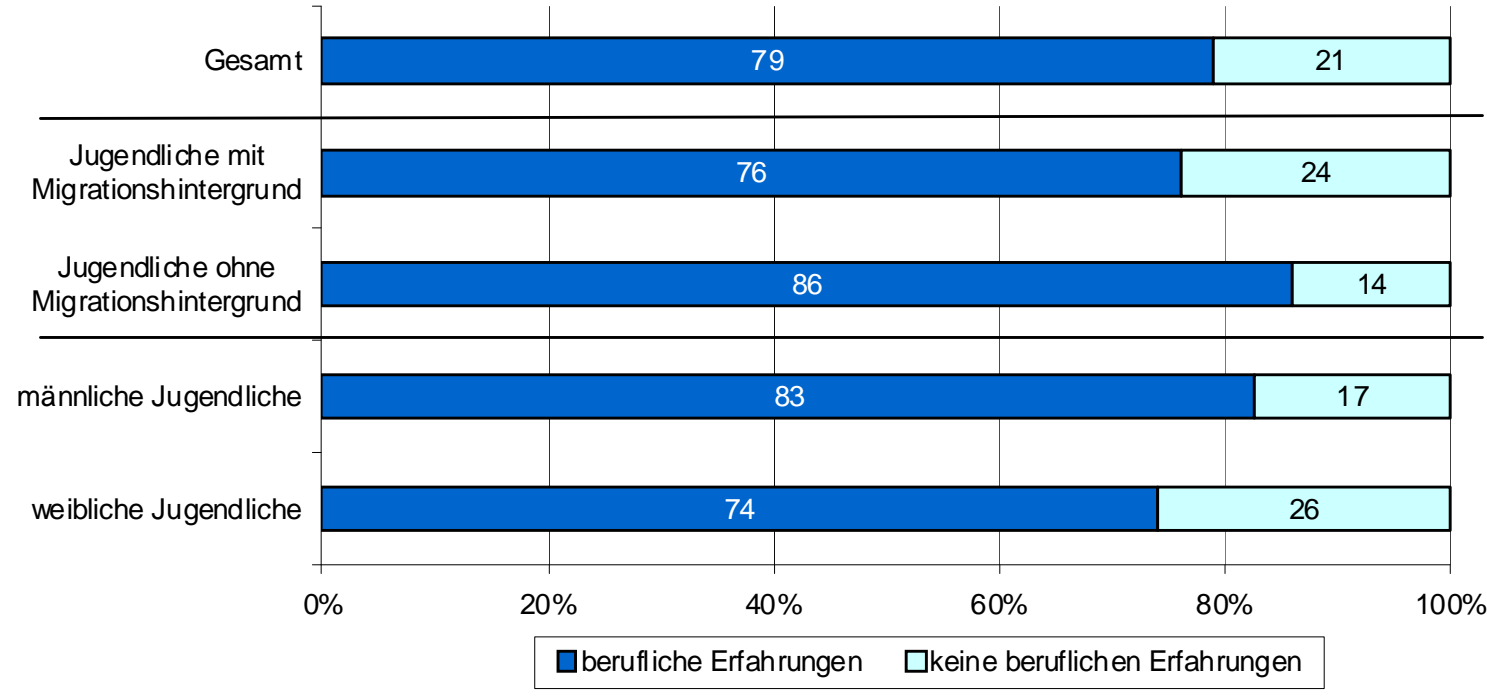

Quelle: KMU FORSCHUNG AUSTRIA, Befragung der jugendlichen AMS-KundInnen, n=431

Rd. zwei Drittel der Jugendlichen mit Arbeitspraxis hat Erfahrungen im Rahmen einer Lehrausbildung in einem Betrieb oder einer Ausbildungseinrichtung sammeln können, insbesondere die österreichstämmigen und männlichen Jugendlichen (76\% und $72 \%$ ), wie aus Grafik 24 ersichtlich.

$\mathrm{Rd} .30 \%$ der befragten Jugendlichen haben bereits in verschiedenen Arbeitsbereichen für einige Monate gearbeitet, insbesondere die migrantischen und die weiblichen Jugendlichen (33\% und $38 \%$ ). Erfahrungen in Hilfsarbeitstätigkeiten haben in erster Linie die männlichen Jugendlichen (14\% vs. $6 \%$ der Mädchen) und die MigrantInnen (12\% vs. $8 \%$ der österreichstämmigen Jugendlichen) gesammelt. Bei mehr als der Hälfte der Jugendlichen hat die (Hilfs-)arbeit weniger als 6 Monate gedauert. Ein Viertel dieser Jugendlichen und insbesondere die Mädchen (30\%) haben jedoch mehr als eine einjährige Berufserfahrung vorzuweisen.

Mehr als ein Zehntel der befragten Jugendlichen hat weiters auch Praktika bzw. Schnuppertage in Unternehmen gemacht. 
Grafik 24 Art der beruflichen Erfahrungen der Jugendlichen, Anteil der befragten Jugendlichen nach Migrationshintergrund und Geschlecht in Prozent
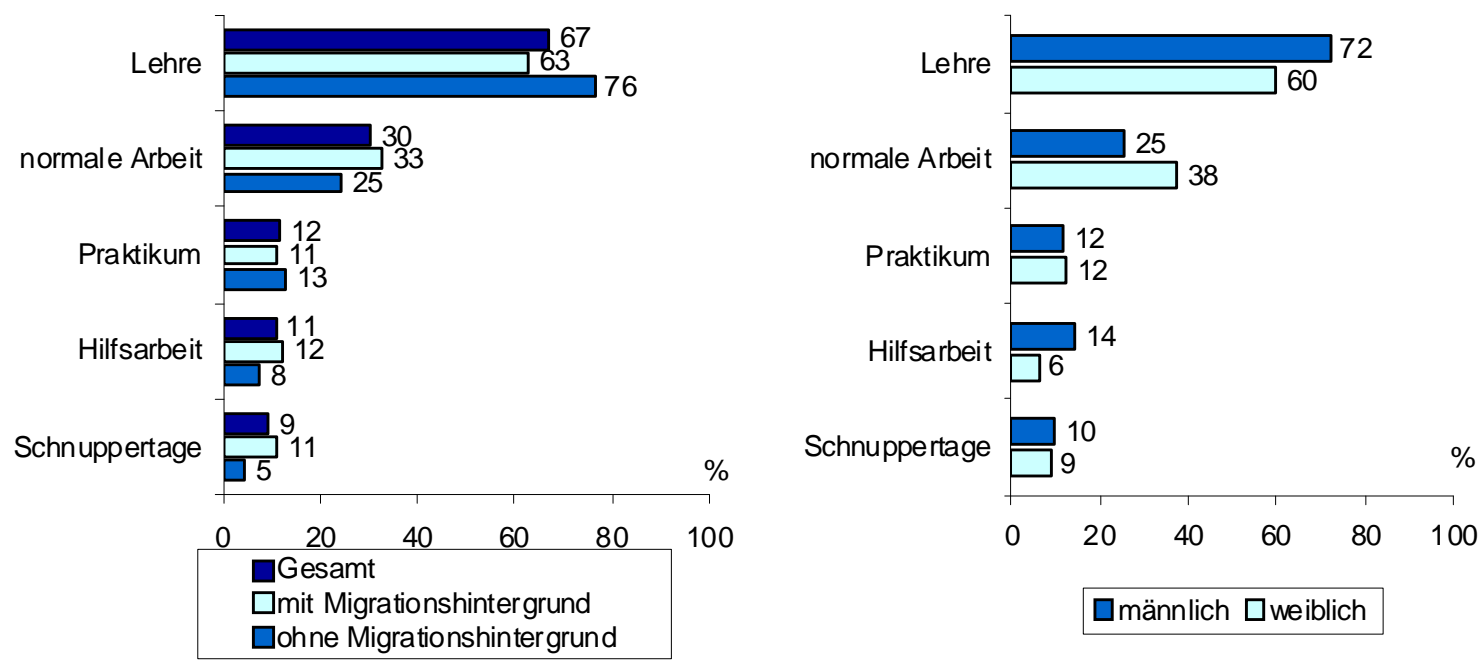

Quelle: KMU FORSCHUNG AUSTRIA, Befragung der jugendlichen AMS-KundInnen, $n=366$, Mehrfachnennungen möglich

Mehr als die Hälfte $(57 \%)$ der befragten Jugendlichen ${ }^{1}$ gab an, bereits einmal eine Lehrausbildung begonnen zu haben, $47 \%$ davon in einem Betrieb und $53 \%$ in einer Ausbildungseinrichtung (siehe Grafik 25). Rd. ein Viertel aller befragten Jugendlichen hat die Lehre abgeschlossen und rd. ein Zehntel befindet sich noch in der Lehrausbildung. Fast die Hälfte dieser Jugendlichen (48 \%) will in diesem Beruf auch in Zukunft tätig sein. Weitere $46 \%$ wollen einen Berufswechsel vornehmen und in einem anderen Bereich in Zukunft arbeiten.

Insgesamt brachen aber fast zwei Drittel (63\%) aller befragten Jugendlichen die Lehrausbildung ab. Die Abbruchsquote ist in den Ausbildungseinrichtungen (74\%) viel höher als in den Betrieben (53 \%). 64 \% aller LehrabbrecherInnen wollen zudem in Zukunft einen anderen Beruf wählen. Ein Drittel jedoch will in demselben Lehrberuf weiter tätig sein bzw. eine neue Lehrstelle suchen.

Der Prozentsatz weicht von dem in Grafik $24 \mathrm{ab}$, da hier eine andere Fragestellung (Lehre ja/nein) vorliegt und bei Grafik 24 Mehrfachnennungen zugelassen wurden. Daher ist die Anzahl der Antworten und damit die Basis für die Errechnung des Prozentsatzes ein andere. 
Grafik 25 Darstellung des Lehrausbildungsverlaufs der Jugendlichen, Anteil der befragten Jugendlichen in Prozent

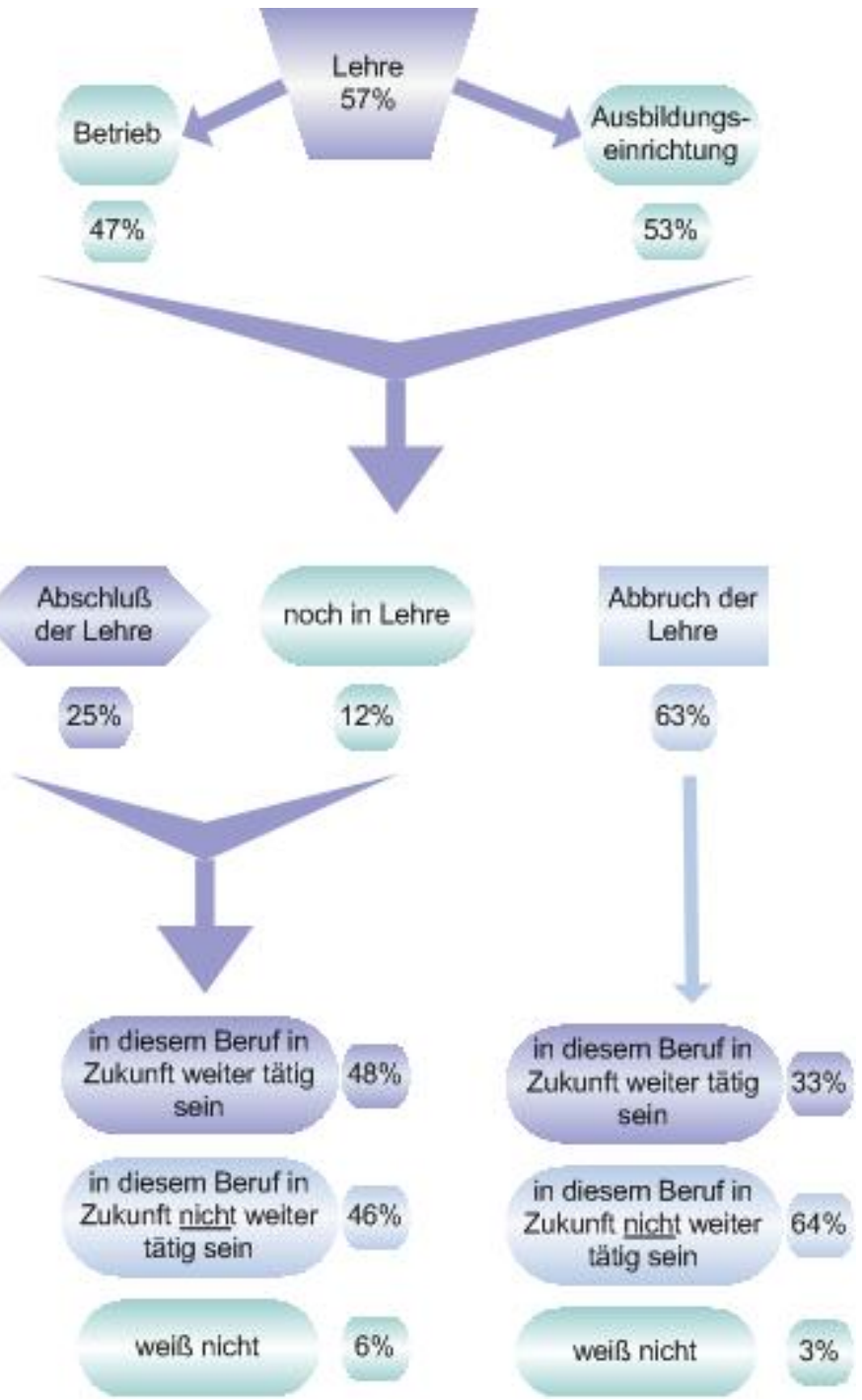

Quelle: KMU FORSCHUNG AUSTRIA, Befragung der jugendlichen AMS-Kundlnnen, n=245 
Unter den befragten Jugendlichen ist die Wahl des Lehrberufs geschlechtsspezifisch unterschiedlich. Mehr als die Hälfte der weiblichen Jugendlichen wählten den Beruf Einzelhandelskauffrau, Friseurin oder Bürokauffrau, wie Tabelle 6 zeigt. Die männlichen Jugendlichen wählten neben den Einzelhandelskaufmann den Beruf des Kochs oder des Kfz-Technikers am häufigsten. Die geschlechtsspezifischen Berufsvorlieben zeigen sich in ähnlicher Weise bei MigrantInnen und Nicht-MigrantInnen (zu den Unterschieden siehe Grafik 26 und Grafik 27).

Tabelle 6 Top-10 Lehrberufe der Jugendlichen, Anteil der befragten Jugendlichen nach Geschlecht in Prozent

\begin{tabular}{l|c|l|c}
\hline \multicolumn{2}{c|}{ weibliche Jugendliche } & \multicolumn{2}{c}{ männliche Jugendliche } \\
\hline Einzelhandelskauffrau & $26 \%$ & Einzelhandelskaufmann & $11 \%$ \\
\hline Friseurin & $15 \%$ & Koch & $11 \%$ \\
\hline Bürokauffrau & $11 \%$ & Kfz-Techniker & $10 \%$ \\
\hline Köchin & $9 \%$ & $\begin{array}{l}\text { Restaurant- und } \\
\text { Gastronomiefachmann }\end{array}$ & $9 \%$ \\
\hline Kosmetik/Fußpflege & $7 \%$ & Elektroinstallationstechniker & $8 \%$ \\
\hline EDV-Kommunikation & $4 \%$ & Maurer & $6 \%$ \\
\hline $\begin{array}{l}\text { Restaurant- und } \\
\text { Gastronomiefachfrau }\end{array}$ & $3 \%$ & Maschinenbautechniker & $5 \%$ \\
\hline $\begin{array}{l}\text { Hotel- und } \\
\text { Gastgewerbeassistentin }\end{array}$ & $3 \%$ & Tischler & $5 \%$ \\
\hline $\begin{array}{l}\text { Pharmazeutisch-kaufmännische } \\
\text { Assistenz }\end{array}$ & $3 \%$ & Sanitär- und Klimatechniker & $4 \%$ \\
\hline \begin{tabular}{l} 
Zahnarztassistentin \\
\hline Quelle: KMU FORSCHUNG AUSTRIA, Befragung der jugendlichen AMS-KundInnen, n=245
\end{tabular} & $\begin{array}{l}\text { Metalltechnik/Metallbearbeitung } \\
\text { S-technik/Schlosser }\end{array}$ \\
\hline
\end{tabular}

Auch beim Verlauf der Lehrausbildung gibt es geschlechtsspezifische Unterschiede: Während $64 \%$ der männlichen Jugendlichen eine Lehre begonnen haben, sind es bei den Mädchen nur $47 \%$, wie Grafik 26 zeigt. Daneben ist der Anteil der Mädchen, die die Lehre in einer Ausbildungseinrichtung begonnen haben, höher (58\% vs. $50 \%$ bei den Buben). Die Lehre positiv abgeschlossen haben die Mädchen als auch die Buben aber im selben Ausmaß ( $r d$. ein Viertel). Die Mädchen scheinen sich bei der Berufswahl ein wenig sicherer zu sein, da rd. $52 \%$ der Mädchen, die noch in der Lehre sind bzw. diese schon abgeschlossen haben, auch in Zukunft diesen Beruf weiter ausüben wollen im Vergleich zu 47 \% der Buben. 
Grafik 26 Darstellung des Lehrausbildungsverlaufs der Jugendlichen, Anteil der befragten Jugendlichen nach Geschlecht in Prozent
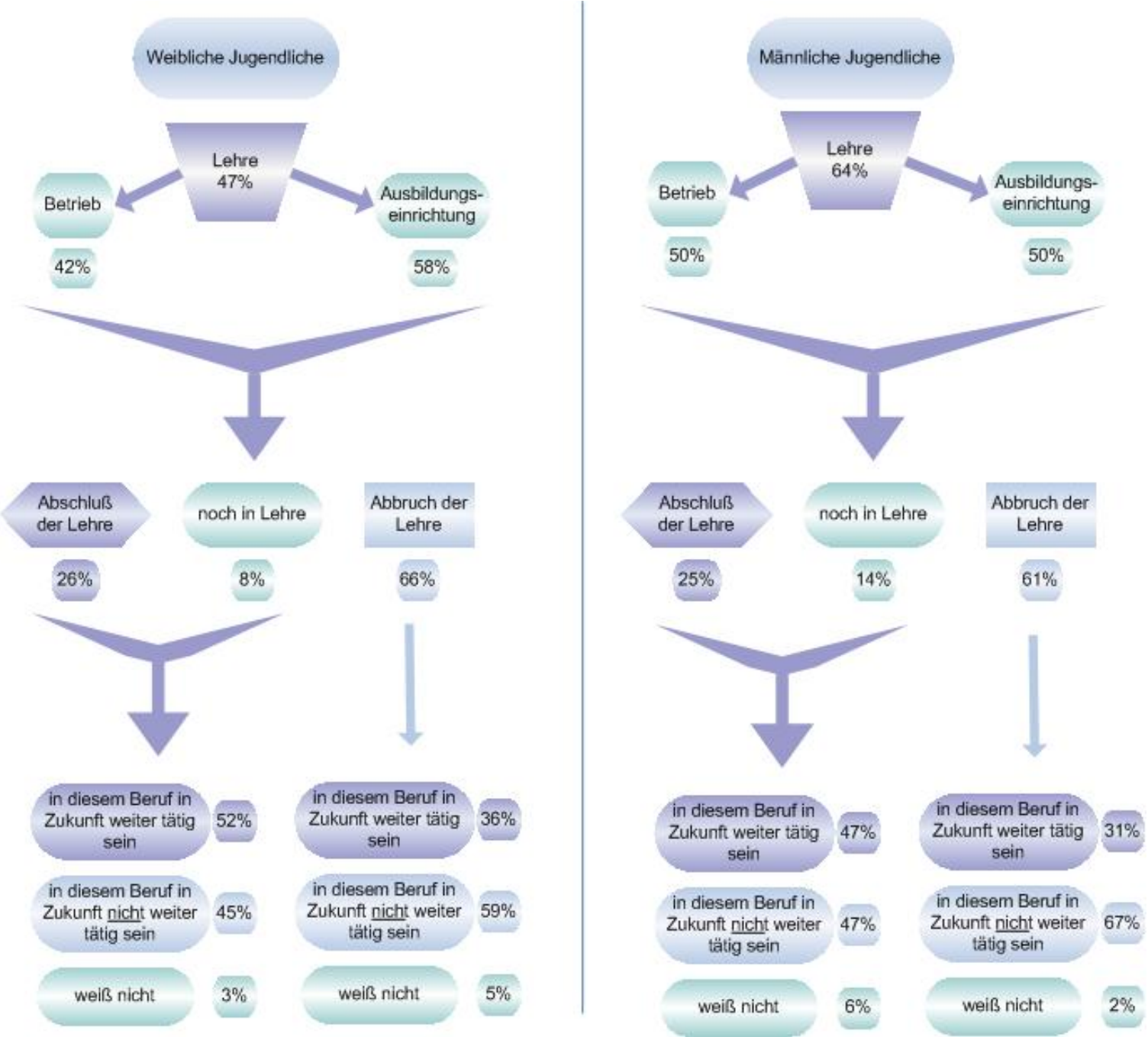

Quelle: KMU FORSCHUNG AUSTRIA, Befragung der jugendlichen AMS-KundInnen, n=245

Zwischen den Jugendlichen mit und ohne Migrationshintergrund bestehen ebenfalls Unterschiede im Bezug auf die Erfahrungen mit der Lehrausbildung. Während $68 \%$ der österreichstämmigen Jugendlichen eine Lehre bereits einmal begonnen haben, sind es unter den migrantischen Jugendlichen nur $53 \%$ (siehe Grafik 27). Letztere haben, wenn sie eine Lehre absolviert haben, diese auch öfters in Ausbildungseinrichtungen gemacht als die österreichstämmigen Jugendlichen (55\% vs. $49 \%$ ). Die Abbruchsquote war unter den Jugendlichen mit Migrationshintergrund auch besonders hoch: Mehr als zwei Drittel (69 \%) der MigrantInnen hat die Ausbildung abgebrochen, bei den österreichstämmigen Jugendlichen waren es „nur“ $51 \%$. Nur hinsichtlich der Berufswahl sind sich MigrantInnen sicherer. 
Grafik 27 Darstellung des Lehrausbildungsverlaufs der Jugendlichen, Anteil der befragten Jugendlichen nach Migrationshintergrund in Prozent

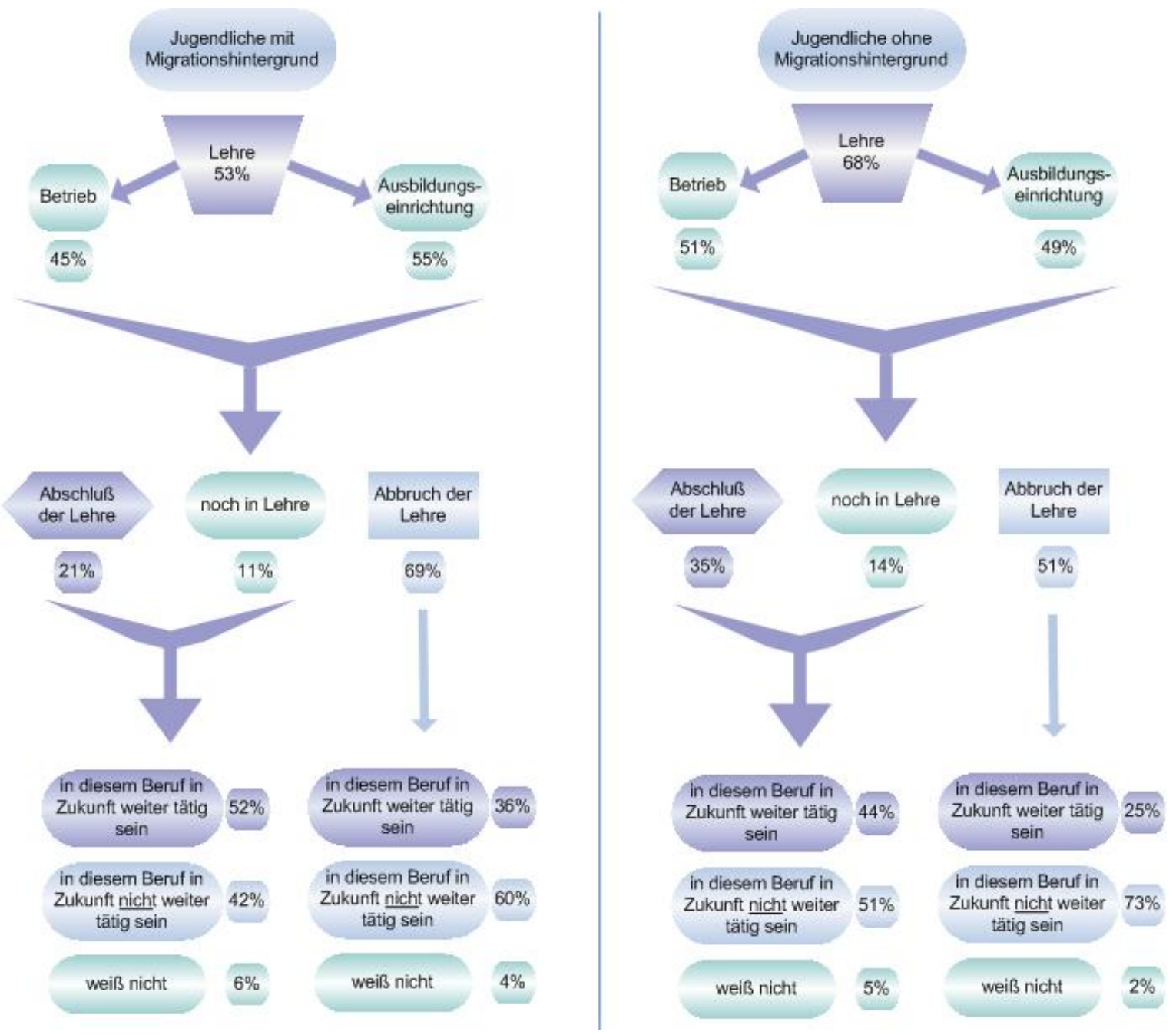

Quelle: KMU FORSCHUNG AUSTRIA, Befragung der jugendlichen AMS-Kundlnnen, n=245

Die Wahl der Lehrberufe bzw. der Berufsbereiche ist je nach Geschlecht und Migrationshintergrund unterschiedlich. Männliche Migranten sind vor allem im Bau/Baunebengewerbe und im Berufsbereich Maschinen/Kfz/Metall sowie Hotel- und Gastgewerbe tätig, wie Grafik 28 zeigt. Die österreichstämmigen Jugendlichen sind neben diesen genannten Berufsbereichen, aber auch vermehrt im Handel/Verkauf, im Büro/ Wirtschaft und Informationstechnologie vorzufinden. 
Grafik 28 Top 7 der Berufsbereiche der männlichen Jugendlichen, Anteil der befragten männlichen Jugendlichen nach Migrationshintergrund in Prozent

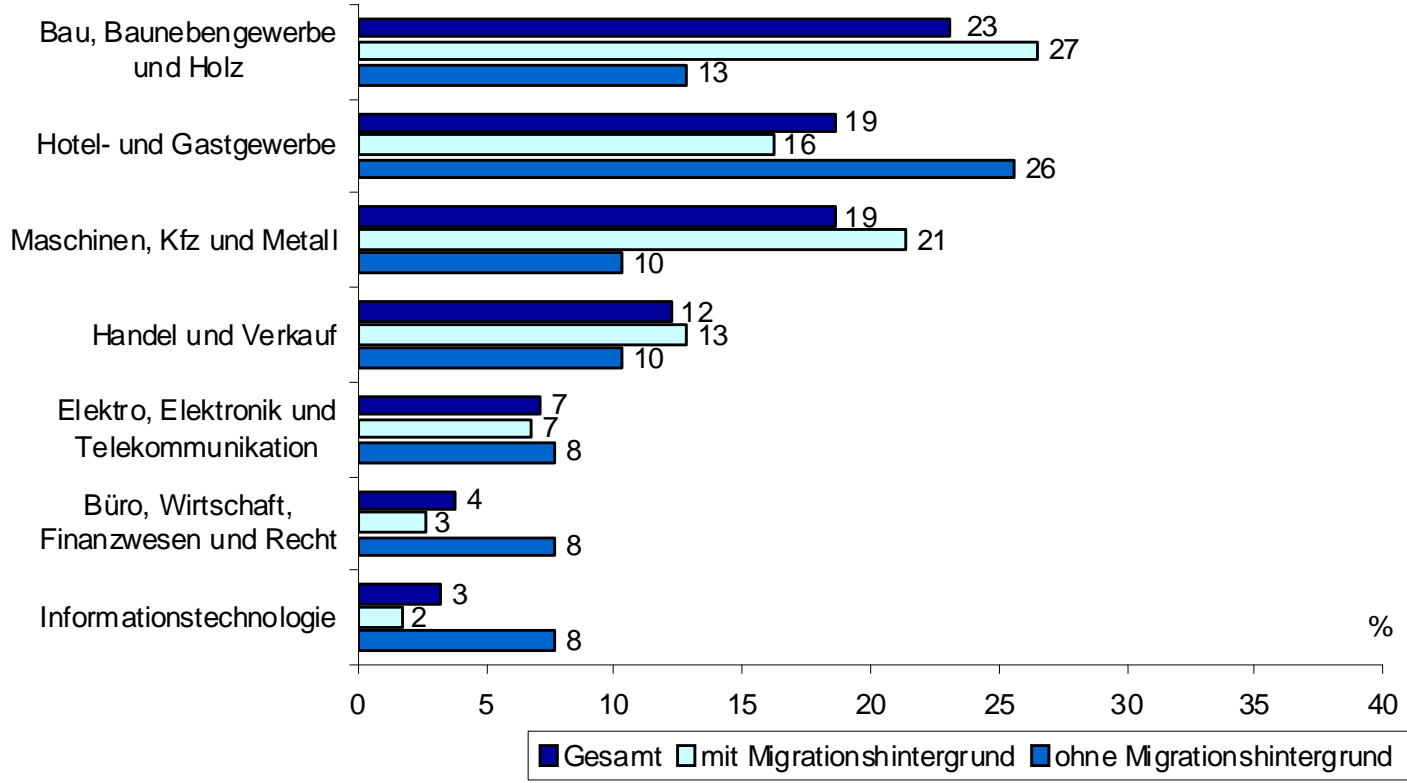

Quelle: KMU FORSCHUNG AUSTRIA, Befragung der jugendlichen AMS-KundInnen, n=245

Auch bei den Mädchen gibt es auch Unterschiede (siehe Grafik 29): Während Migrantinnen in erster Linie im Handel/Verkauf und im Hotel- und Gastgewerbe vorzufinden sind, wählen die österreichstämmigen Mädchen die Körper- und Schönheitspflege noch vor dem Handel/Verkauf. Wie auch bei den Buben sind letztere auch häufiger im Berufsbereich Büro/Wirtschaft tätig als die Mädchen mit Migrationshintergrund. Dies ist sicherlich auf die manchmal mangelhaften Deutschkenntnisse der MigrantInnen zurückzuführen, die für einen Bürojob nicht ausreichen.

Grafik 29 Top-7 der Berufsbereiche der weiblichen Jugendlichen, Anteil der befragten weiblichen Jugendlichen nach Migrationshintergrund in Prozent

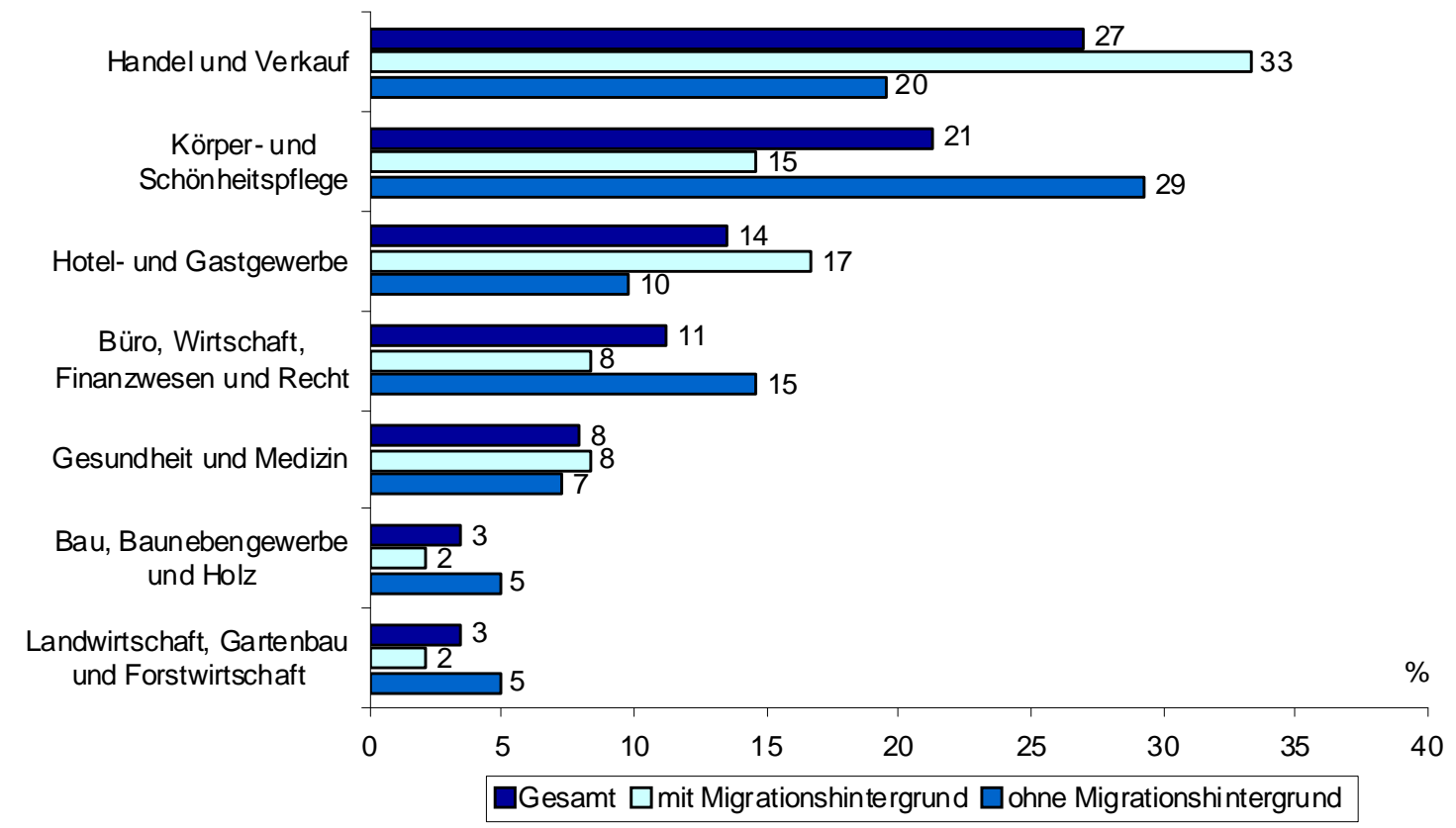

Quelle: KMU FORSCHUNG AUSTRIA, Befragung der jugendlichen AMS-KundInnen, n=245 


\subsection{Berufswünsche}

In Hinblick auf die Berufswünsche der Jugendlichen, die noch keinen Beruf gewählt haben, besteht auch wie bei den anderen Jugendlichen eine starke geschlechtsspezifische Segmentierung, wie Grafik 30 zeigt. Während die Buben zu technischen und handwerklichen Berufe tendieren (wie z. B. Berufsbereiche wie Maschinen/Kfz/Metall oder Bau), bevorzugen Mädchen den Handel/Verkauf oder Ausbildungen im Bürobereich oder im Bereich der Gesundheit/Medizin.

Grafik 30 Wunschberufsbereiche der Jugendlichen, Anteil der befragten Jugendlichen nach Geschlecht in Prozent

männliche Jugendliche

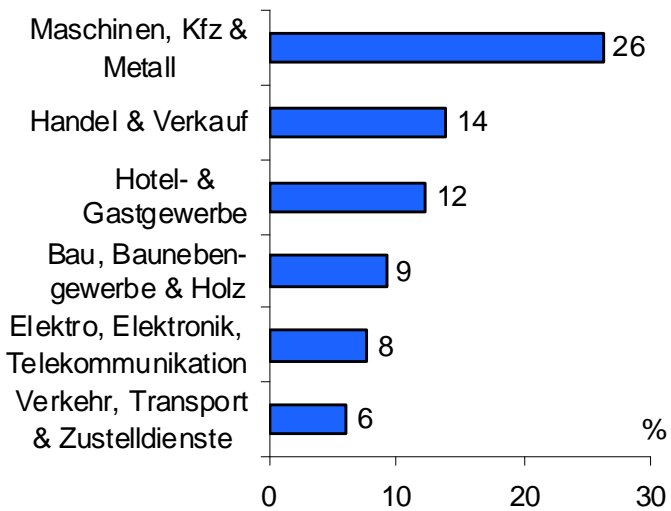

weibliche Jugendliche

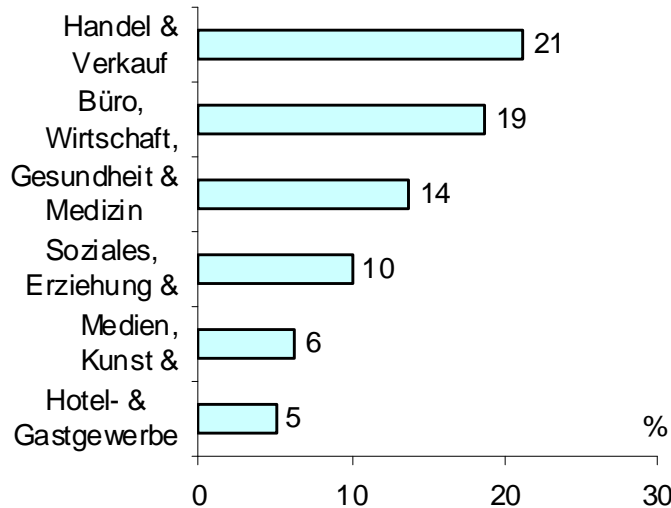

Quelle: KMU FORSCHUNG AUSTRIA, Befragung der jugendlichen AMS-KundInnen, n=145

Wie sind die Jugendlichen zu ihrer Berufswahl gekommen? Mehr als die Hälfte der befragten Jugendlichen gibt an, dass sie sich alleine entschieden haben (siehe Grafik 31). Rd. ein Fünftel hat sich aber mit den Eltern beraten, insbesondere die österreichstämmigen Jugendlichen. Rd. ein Zehntel wurde von FreundInnen beeinflusst. Rd. $8 \%$ der migrantischen Jugendlichen haben außerdem von BeraterInnen Hilfe in Anspruch genommen. Weitere $4 \%$ der Jugendlichen haben sich von LehrerInnen beraten lassen. Neben den Eltern nimmt auch die weitere Verwandtschaft Einfluss auf die Berufswahl, wie in den Fokusgruppen erläutert wurde: „Mein Onkel ist auch Elektriker. Ich habe mich auch bei seiner Firma beworben." 


\section{Grafik $31 \quad$ Hilfe bei der Berufsfindung der Jugendlichen, Anteil der befragten} Jugendlichen nach Migrationshintergrund in Prozent

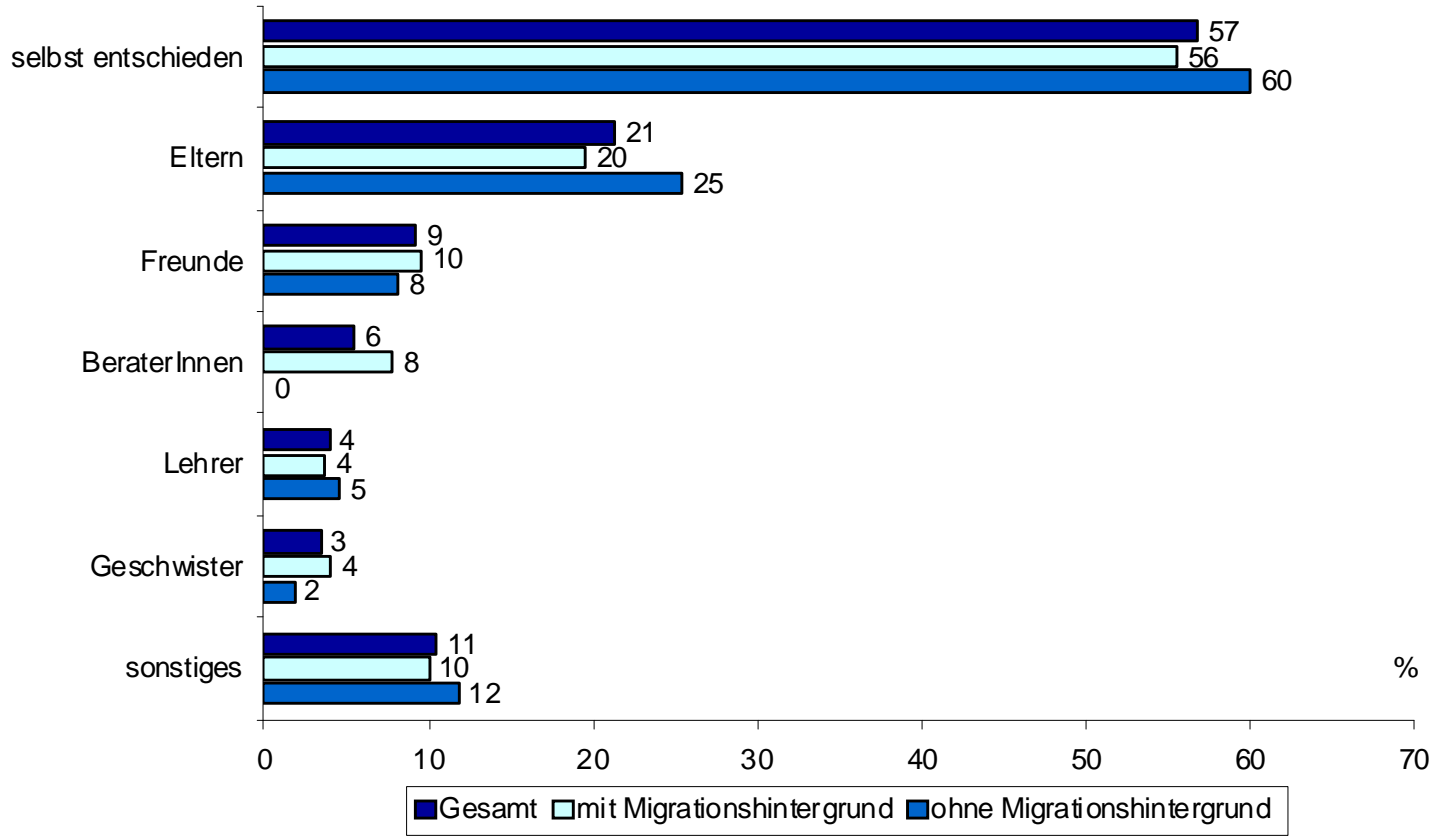

Quelle: KMU FORSCHUNG AUSTRIA, Befragung der jugendlichen AMS-KundInnen, n=380, Mehrfachnennungen möglich

Als konkrete Informationsquellen für die Berufswahl nannten rd. ein Drittel der Jugendlichen das AMS, insbesondere die MigrantInnen (37\% vs. $27 \%$ der österreichstämmigen Jugendlichen), wie aus Grafik 32 ersichtlich. Die österreichstämmigen Jugendlichen informierten sich hingegen häufiger im Internet (35\% vs. $24 \%$ der MigrantInnen). Berufsorientierungskurse wurden in erster Linie von den MigrantInnen als Informationsquelle genutzt (16\% vs. $6 \%)$. Weitere Informationsquellen waren die Familie bzw. der Bekanntenkreis (12\%), die Schule (6\%), das BIZ (3\%) oder Berufsinformationsberatungen bzw. -messen (2\%). Rd. ein Fünftel der Befragten und insbesondere die österreichstämmigen Jugendlichen (24\% vs. $17 \%$ der MigrantInnen) haben sich nirgends informiert. 
Grafik 32 Informationsquellen der Jugendlichen für die Berufswahl, Anteil der befragten Jugendlichen nach Migrationshintergrund in Prozent

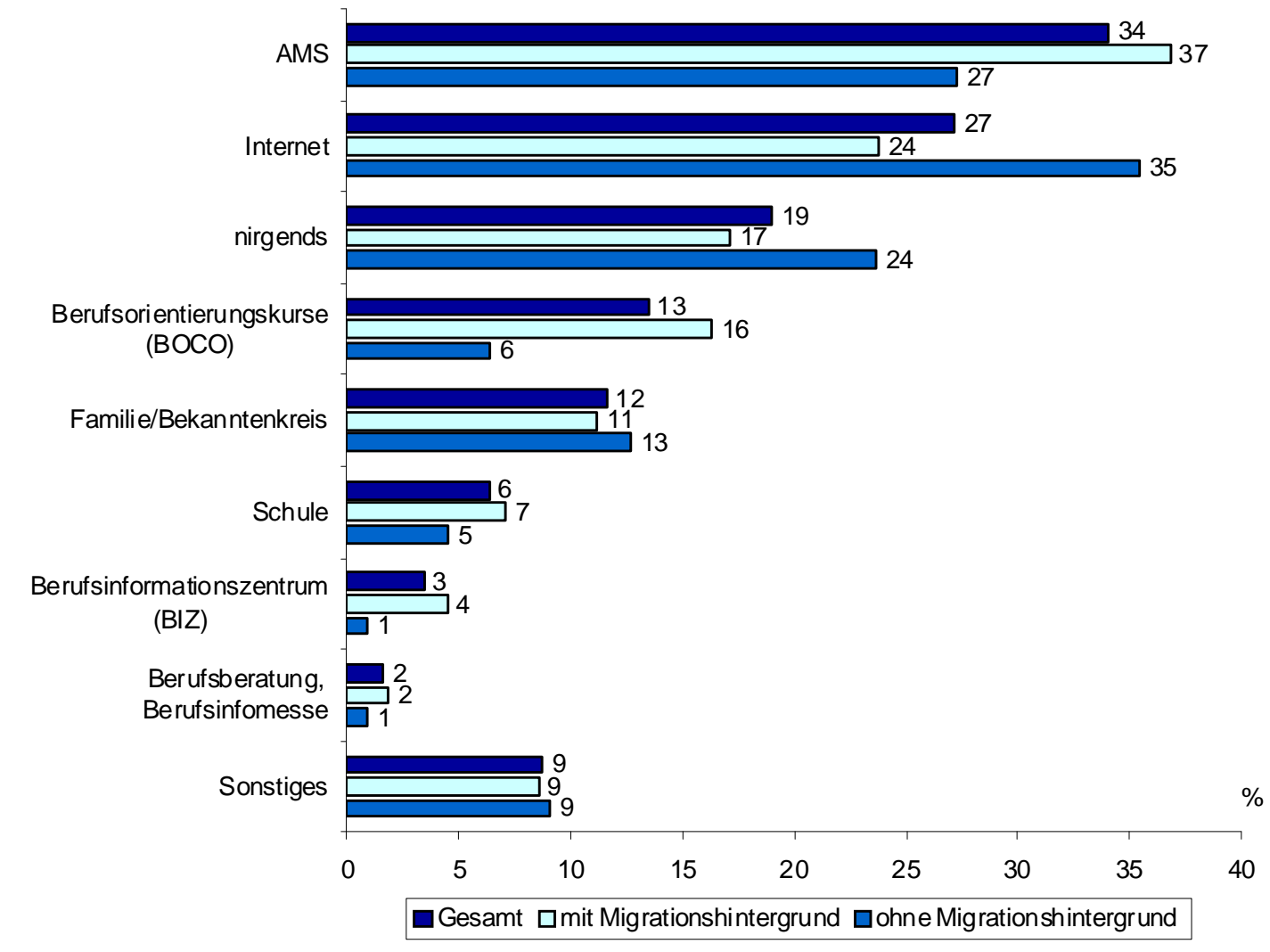

Quelle: KMU FORSCHUNG AUSTRIA, Befragung der jugendlichen AMS-KundInnen, $n=379$, Mehrfachnennungen möglich

Rd. ein Drittel der befragten Jugendlichen gab an, das sie das Berufsinformationszentrum (BIZ) im AMS besucht haben. In erster Linie jedoch nur einmal (23\% vs. $9 \%$ öfters). Rd. die Hälfte jener Jugendlichen (49\%), die das BIZ besucht haben, hat dies mit der Schule gemacht. Für jene Jugendlichen, die das BIZ besucht haben, war es für mehr als zwei Drittel (69\%) hilfreich und insbesondere für die migrantischen Jugendlichen (73\% vs. $59 \%$ der österreichstämmigen Jugendlichen).

\subsection{Die Arbeitsmarktintegration beeinflussende Faktoren}

Die Jugendlichen sind bemüht, durch eigene Bewerbungsaktivitäten möglichst rasch eine freie Lehrstelle oder einen Arbeitsplatz zu finden. $91 \%$ der Befragten suchen selbstständig nach freien Stellen am Arbeitsmarkt. Bei der Lehrstellen- oder Arbeitsplatzsuche greift der Großteil der jugendlichen AMS-KundInnen (84 \%) auf das Internet zurück, wie Grafik 33 zeigt. Für Jugendliche ohne Migrationshintergrund hat das Internet für die Stellensuche noch eine höhere Relevanz als für MigrantInnen (93\% vs. $80 \%$ ). Beinahe ein Drittel (32\%) zieht Inserate in Zeitungen und Zeitschriften für die Suche nach beruflichen Möglichkeiten heran, wobei österreichische Jugendliche diese Suchoption häufiger in Anspruch nehmen als KundInnen mit Migrationshintergrund (38 \% vs. 29 \%). Ein Fünftel versucht über Freunde und Bekannte einen Job zu finden und $15 \%$ sprechen persönlich bei potenziellen ArbeitgeberInnen vor, um direkt ihre Beschäftigungschancen auszuloten. Insbesondere Jugendliche mit Migrationshintergrund schätzen den persönlichen Besuch bei Unternehmen und wenden sich bei der 
Suche nach Arbeitsmöglichkeiten häufiger an den Freundes- und Bekanntenkreis als KundInnen ohne Migrationshintergrund. Dies zeigt einerseits die stärkere Vernetzung in der Community, andererseits können über persönliche Vorsprache und Vermittlung im Bekanntenkreis formalisierte Bewerbungsprozesse umgangen werden. Ein Zehntel der Jugendlichen versucht über das familiäre Umfeld eine freie Lehr- oder Arbeitsstelle zu finden, dies trifft wiederum etwas häufiger auf KundInnen aus Österreich als auf MigrantInnen zu. Dafür könnten u. a. auch die unterschiedlichen Arbeitsschwerpunkte der Eltern der beiden Zielgruppen ausschlaggebend sein.

Grafik 33 Informationsquellen der Jugendlichen für die Arbeitssuche, Anteil der befragten Jugendlichen nach Migrationshintergrund in Prozent

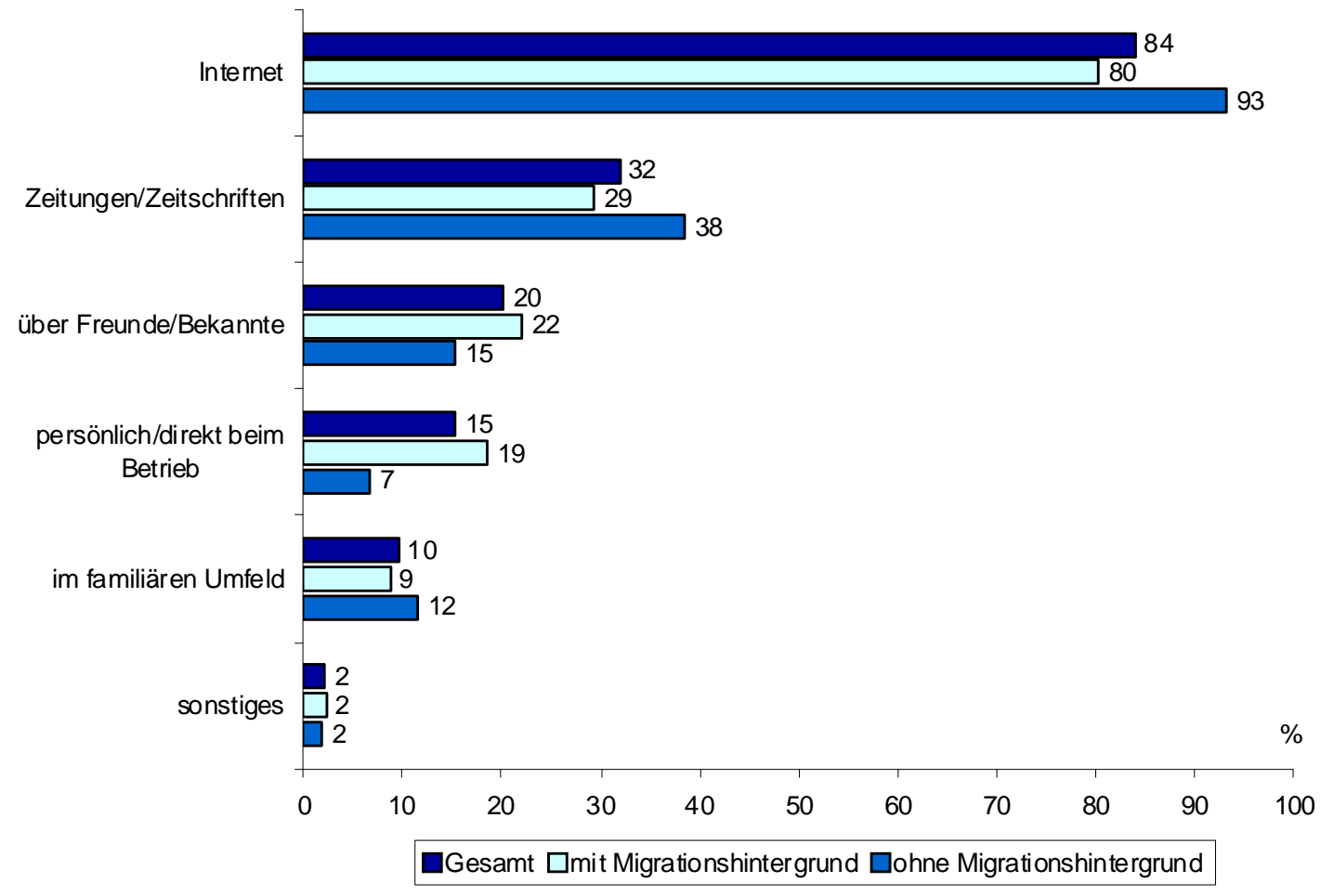

Quelle: KMU FORSCHUNG AUSTRIA, Befragung der jugendlichen AMS-KundInnen, $\mathrm{n}=367$, Mehrfachnennungen möglich

Obwohl das Internet für die Jugendlichen das bedeutendste Werkzeug für die Stellensuche darstellt, greift nur etwas mehr als ein Drittel der KundInnen (36\%) auf die Computer, die im AMS zur Verfügung stehen, zurück. Beinahe ein Viertel davon nutzt die EDV-Infrastruktur des AMS öfters. Insbesondere Jugendliche mit Migrationshintergrund nehmen die PCs des AMS insgesamt häufiger in Anspruch als KundInnen aus Österreich (40\% vs. $25 \%$ ). Auch männliche Jugendliche machen davon eher Gebrauch als Mädchen (39\% vs. $32 \%$ ). Die Mehrheit der KundInnen (64\%) nimmt jedoch das EDV-Angebot nicht in Anspruch, da sie bevorzugt am eigenen PC nach freien Stellen im Internet sucht.

Trotz zahlreicher Informationsmöglichkeiten wird der Bewerbungsprozess bei einigen KundInnen dadurch erschwert, da sie bereits mit grundlegenden Anforderungen, wie z. B. der Erstellung ihrer Bewerbungsunterlagen, überfordert sind. Dieses Problem wird auch teilweise bei den Jugendlichen, die im Rahmen der Fokusgruppen befragt wurden, thematisiert. Ein Mädchen fühlte sich beim Bewerbungsprozess alleine gelassen: „Das AMS hat mir überhaupt nicht geholfen. Ich habe jeden Tag geweint, die machen 
nichts. Du musst das [Bewerbung] schreiben und schicken, die helfen nicht, die machen überhaupt nichts. Eine andere Jugendliche hat ähnliche Erfahrungen gemacht: „Die vom AMS sagen nicht, wie man einen Lebenslauf schreiben soll, die wollen uns nur weg haben. Die kontrollieren nur." Damit wird deutlich, dass einige Jugendliche umfassendere Unterstützung beim Bewerbungsprozess, wie beim Erstellen des Lebenslaufs oder von Bewerbungsschreiben, sowie auch bei Aufnahmeprüfungen und Anmeldungsprozessen, benötigen, die die AMS-BeraterInnen nicht bieten können. Daher ist eine intensivere Betreuung in speziellen Kursen oder von Beratungseinrichtungen erforderlich. Aber auch die MitarbeiterInnen im BerufsInfoZentrum (BIZ) sind bemüht, die Jugendlichen zu beraten, wie ein Bewerbungsschreiben und ein Lebenslauf zu gestalten sind.

\section{Arbeitsmarktrelevante Fähigkeiten und Kenntnisse}

Die Jugendlichen sind sich in hohem Maße darüber bewusst, welche Fähigkeiten und Kenntnisse die Arbeitsmarktintegration erleichtern. Fast die Hälfte der KundInnen sieht in einer guten Ausbildung (Lehr- oder Schulabschluss) einen entscheidenden Faktor, um bei der Arbeitssuche erfolgreich zu sein (siehe Grafik 34). Vor allem Mädchen (50\%) und Jugendliche mit Migrationshintergrund (49\%) sehen einen guten Ausbildungshintergrund für den Bewerbungsprozess als vorteilhaft an. Auch bei der Erhebung im Jahr 2007 wiesen $44 \%$ der befragten MigrantInnen auf die Bedeutung einer guten Ausbildung hin (vgl. Heckl et al., 2007). Etwa ein Fünftel (21\%) ist der Meinung, dass der eigene (Arbeits-)Wille, die Motivation, das Engagement und die Eigeninitiative bei der Stellensuche ausschlaggebend sind und weisen darauf hin, dass beim Bewerbungsprozess auch Geduld und Durchhaltevermögen gefragt sind. Im Vergleich zur Erstuntersuchung im Jahr 2007 haben Arbeitswille und Eigeninitiative bei den MigrantInnen (22\%) deutlich an Relevanz gewonnen, da damals nur $7 \%$ der Jugendlichen mit Migrationshintergrund explizit den eigenen Willen erwähnten (vgl. Heckl et al., 2007).

Weitere bedeutende Faktoren für eine erfolgreiche Arbeitsmarktintegration sind laut Auskunft von jeweils rd. einem Fünftel der AMS-KundInnen gute Deutschkenntnisse und ein positiver Hauptschulabschluss. Insbesondere Jugendliche mit Migrationshintergrund $(23 \%)$ erachten gute Kenntnisse der deutschen Sprache für den Vermittlungserfolg als wesentlich, aber auch für $13 \%$ der KundInnen aus Österreich sind gute Deutschkenntnisse - vor allem auf schriftlicher Ebene - ein entscheidendes Erfolgskriterium. Für $14 \%$ der Befragten sind verschiedene Soft Skills und Arbeitstugenden, wie z. B. Selbstbewusstsein, kommunikative Fähigkeiten, Freundlichkeit, Pünktlichkeit, Zuverlässigkeit, Teamorientierung, relevant, um am Arbeitsmarkt Fuß zu fassen. Jugendliche aus Österreich messen diesen Eigenschaften deutlich mehr Bedeutung bei als MigrantInnen (24\% vs. $10 \%$ ). Ein Mädchen aus einer Fokusgruppe erzählt, was ihrer Meinung nach im Bewerbungsprozess relevant ist: „Die Arbeitgeber wollen einen verlässlichen Mitarbeiter. Die Unternehmer erwarten Zuverlässigkeit, Pünktlichkeit. Sie schauen sich die Zeugnisse an, in welchen Fächern man gut war. Die Bewerbung schauen sie sich auch genau an, wie man das schreibt." Ein weiterer Vorteil im Bewerbungsprozess ist, wenn die KundInnen bereits eine gewisse berufliche Praxis bei Arbeits- oder Praktikumsstellen erwerben konnten.

Auch ein gepflegtes Äußeres, ein gutes, selbstsicheres Auftreten und eine gute Selbstpräsentation kann laut Auskunft von einem Zehntel der Jugendlichen im Bewerbungsprozess hilfreich sein. Für KundInnen ohne Migrationshintergrund ist das Aussehen und Auftreten sogar noch etwas wichtiger als für Jugendliche mit migrantischem Hintergrund (14\% vs. $9 \%$ ). $10 \%$ aller KundInnen sind der Meinung, dass gut vorbereitete 
Bewerbungsunterlagen, eine gute Orientierung beim Bewerbungsprozess, Unterstützung bei der Jobsuche sowie Informationen über den zukünftigen Arbeitsplatz eine erfolgreiche Arbeitsmarktintegration beschleunigen. Als weitere Kriterien, die den Einstieg in den Arbeitsprozess erleichtern, werden gute Noten und Abschlusszeugnisse, das Interesse an der Arbeit, weitere Sprachkenntnisse sowie die Kenntnisse und Fähigkeiten im gewählten Beruf ebenso wie allgemeine Intelligenz genannt.

Die Jugendlichen schätzen vor allem ihre weiteren Sprachkenntnisse, die durchaus einen Bewerbungsvorteil darstellen könnten, als nicht sehr relevant ein, da sie bisher diesbezüglich vorwiegend negative Erfahrungen am Arbeitsmarkt machten, wie ein junger Mann bei einer Fokusgruppe erzählt: „Keiner weiß es zu schätzen, dass wir mehrere Sprachen können." Ein Grund dafür könnte sein, dass sie die Sprache nur mündlich und nicht schriftlich beherrschen, wie auch folgende Aussagen verdeutlichen: „Auf Türkisch habe ich [in meinem Leben] nur einen Absatz gelesen. Ich glaube nicht, dass ich in der Türkei [von den Sprachkenntnissen her] arbeiten könnte. " Ein anderer meint: „Ich könnte es lernen wenn ich will, aber was brauch' ich die Schrift. Ich bin ja nicht in Serbien, ich bin hier [in Österreich]". Die Einsicht, dass schriftliche Fremdsprachenkenntnisse ein Vorteil sein könnten, ist kaum gegeben, wie ein Jugendlicher berichtet: „Aber wenn ich in Österreich arbeite, wozu brauch' ich die arabische Schrift."

Grafik 34 Erforderliche Fähigkeiten, Kenntnisse und Rahmenbedingungen, um eine Arbeit zu finden, Anteil der befragten Jugendlichen nach Migrationshintergrund in Prozent

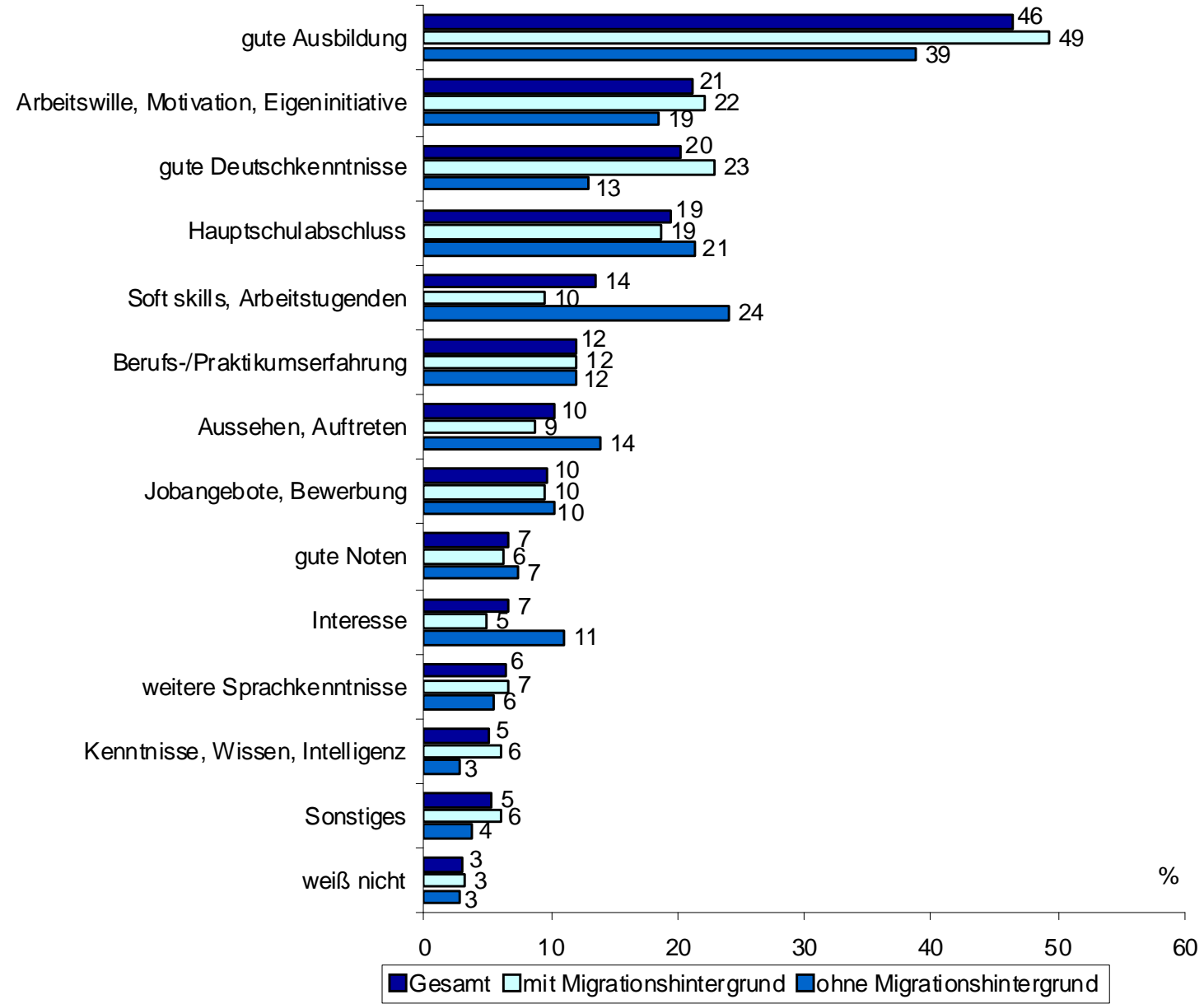

Mehrfachnennungen möglich

Quelle: KMU FORSCHUNG AUSTRIA, Befragung der jugendlichen AMS-KundInnen, $\mathrm{n}=392$, 


\section{Schwierigkeiten am Arbeitsmarkt}

Etwa $80 \%$ der befragten Jugendlichen haben Schwierigkeiten, sich am Arbeitsmarkt zu integrieren, wie aus Grafik 35 ersichtlich. Mädchen (82 \%) und MigrantInnen (81 \%) nehmen etwas häufiger Probleme bei der Integration in den Arbeitsmarkt wahr als Burschen (76 \%) bzw. KundInnen aus Österreich (72\%). Damit hat sich der Anteil der befragten MigrantInnen, die mit Schwierigkeiten konfrontiert sind, im Vergleich zu 2007, wo dies etwa zwei Drittel der befragten KundInnen mit Migrationshintergrund angaben, erhöht (vgl. Heckl et al., 2007).

Die Jugendlichen beklagen in erster Linie, dass zu wenige Lehrstellen oder Arbeitsplätze verfügbar sind bzw. dass zu viele BewerberInnen um eine Stelle konkurrieren. Dadurch verlängert sich die Arbeitssuche teilweise erheblich. Vor allem Mädchen (19\%) sehen sich einer großen Konkurrenz und wenig freien Stellen gegenüber, während dies nur auf $12 \%$ der männlichen Zielgruppe zutrifft. Dieses Problem wird auch bei den Fokusgruppen erläutert: „Es gibt mehrere Lehrstellensuchende, der Arbeitgeber will auch nicht jeden nehmen." Ebenso stellen fehlende Schul- und Ausbildungsabschlüsse (insbesondere der Hauptschule und der Lehre) und schlechte Noten für $15 \%$ der Jugendlichen eine erhebliche Vermittlungsbarriere dar. Jugendliche aus Österreich führen diese Ausbildungsdefizite häufiger als Problem an als MigrantInnen (19\% vs. $13 \%$ ). Ein Zehntel der Befragten gibt an, dass sie zu faul sind und innen die Motivation bzw. Lust fehlt, sich der anstrengenden Suche nach einer Arbeit oder Lehrstelle zu widmen. Diese Motivationsprobleme treffen vorwiegend auf männliche Jugendliche (15\%) zu, während dies bei Mädchen deutlich seltener der Fall ist (5\%).

Einigen Jugendlichen erscheinen die gesuchten Anforderungen bei den Stellenanzeigen zu hoch bzw. nicht passend und sie beklagen sich, dass sie von den Unternehmen keine Rückmeldungen oder nur Absagen erhalten. Diese Unstimmigkeiten und Ablehnungen werden von Mädchen eher als problematisch erlebt als von Burschen. Auch die Jugendlichen berichten in den Fokusgruppen von zahlreichen Frustrationserlebnissen: "Ich habe schon viele Bewerbungen verschickt. Ich habe viele Absagen erhalten, manche Unternehmen antworten, manche gar nicht." Ein anderer Jugendlicher erzählt: „Ich habe schon Praktika bei 6 Firmen gemacht und habe nur gehört: „Wir melden uns, wir melden uns, wir melden uns."

Außerdem haben manche Schwierigkeiten, den für sie passenden Beruf zu finden, Bewerbungsschreiben aufzusetzen und ein gutes Vorstellungsgespräch zu führen, da sie mit Nervosität und Schüchternheit zu kämpfen haben. Des Weiteren erschweren diverse persönliche Probleme - insbesondere Krankheiten und Straffälligkeiten - die Integration am Arbeitsmarkt, wobei dies vor allem KundInnen ohne Migrationshintergrund bzw. männliche Jugendliche zutrifft. Auch migrantenspezifische Barrieren, wie unzureichende Deutschkenntnisse, das Tragen eines Kopftuchs sowie Diskriminierung und Vorurteile von Seiten der potenziellen Arbeitgeberlnnen, werden insbesondere bei MigrantInnen bei der Arbeitssuche wirksam.

Ein weiteres Hemmnis ist, wenn die Jugendliche über keinerlei bzw. nicht ausreichend Berufserfahrung verfügen. Personen mit Migrationshintergrund sowie Mädchen haben etwas häufiger auf Grund mangelnder beruflicher Erfahrung Schwierigkeiten bei der Integration in den Arbeitsmarkt. 
Grafik 35 Persönliche Probleme am Arbeitsmarkt, Anteil der befragten Jugendlichen nach Migrationshintergrund in Prozent

zu wenig (Lehr-)Stellen, zu viele BewerberInnen

fehlende Ausbildung, schlechte Noten

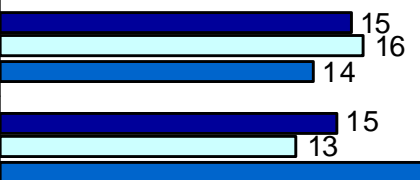

Faulheit, keine Lust

Anforderungen zu hoch, nur negative Rückmeldungen

Probleme bei Berufswahl, Bewerbungsprozess
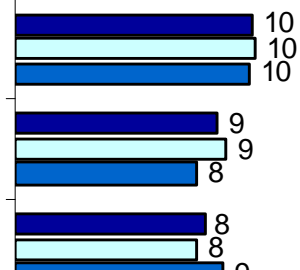

Persönliche Gründe (Krankheit, Haft) mangelnde Deutschkenntnisse, Kopftuch, Diskriminierung

keine Berufserfahrung
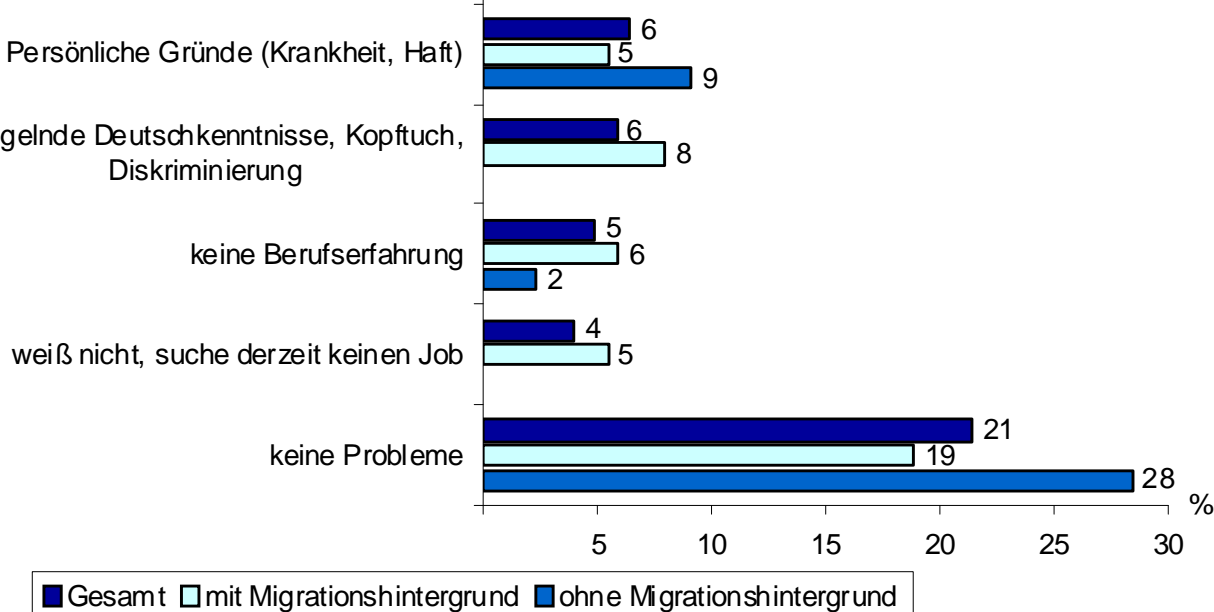

Quelle: KMU FORSCHUNG AUSTRIA, Befragung der jugendlichen AMS-KundInnen, $\mathrm{n}=327$

Trotz diverser Probleme bei der Suche nach freien Arbeitsplätzen oder Lehrstellen hat die Mehrheit der befragten Jugendlichen (60\%) nicht den Eindruck, dass sie es beim Bewerbungsprozess schwieriger haben als andere junge Menschen (siehe Grafik 36). Rd. ein Drittel (34\%) fühlt sich jedoch benachteiligt. Dies trifft auf Jugendliche mit Migrationshintergrund häufiger zu als auf österreichische KundInnen (36 \% vs. 30 \%). 
Grafik 36 Gefühl der Jugendlichen, dass sie bei der Arbeits-/Lehrstellensuche mehr Schwierigkeiten haben als andere, Anteil der befragten Jugendlichen nach Migrationshintergrund in Prozent

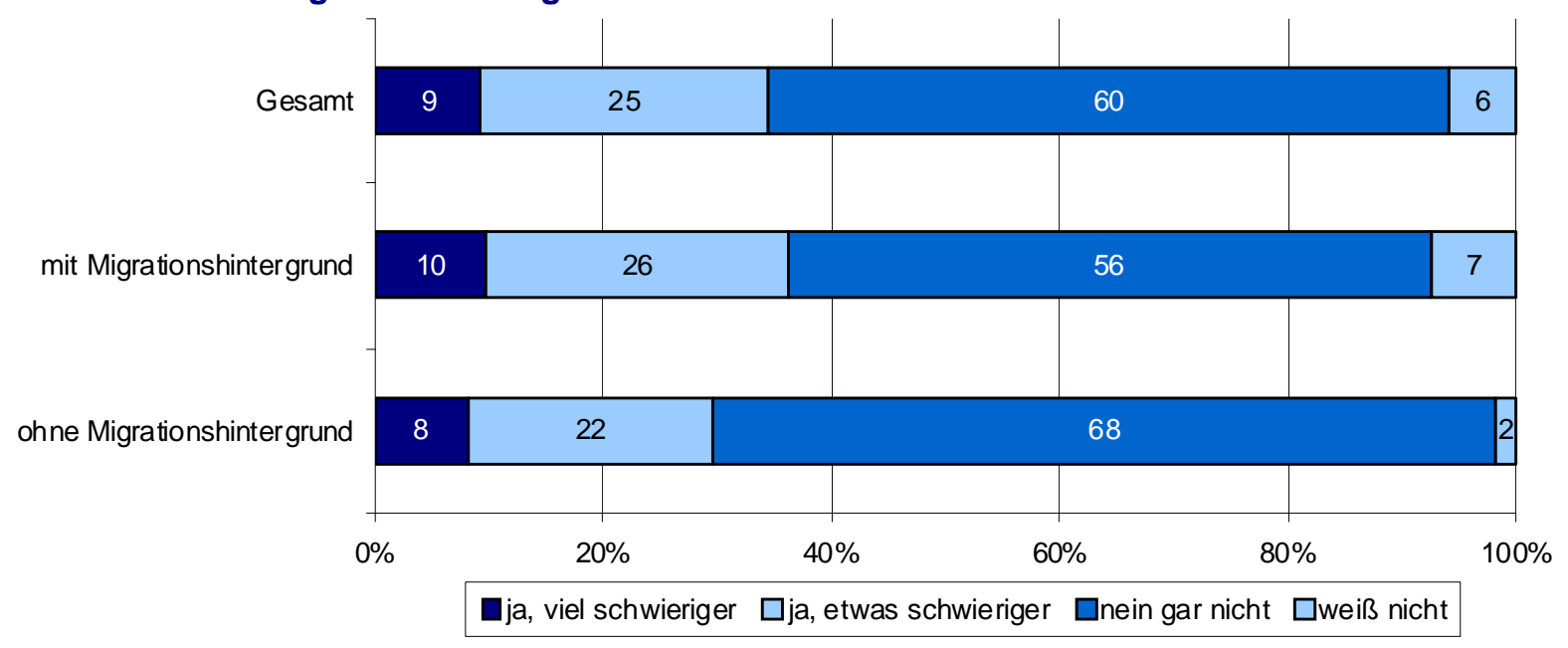

Quelle: KMU FORSCHUNG AUSTRIA, Befragung der jugendlichen AMS-KundInnen, $\mathrm{n}=407$

Die Jugendlichen, die das Gefühl haben, mit mehr Schwierigkeiten bei der Arbeitsmarktintegration konfrontiert zu sein als andere, führen dies in erster Linie auf ihre fehlenden Ausbildungsabschlüsse und schlechte Noten zurück (37\%), wobei diese vor allem KundInnen aus Österreich (44\%) als Vermittlungsbarriere wahrnehmen. Etwa ein Fünftel (21\%) führt Benachteiligungen an, die in Zusammenhang mit dem Migrationshintergrund stehen, wie z. B. mangelnde Sprachkenntnisse, das Kopftuch, Diskriminierungen oder eine fehlende österreichische Staatsbürgerschaft. Auch diverse persönliche Probleme (wie körperliche Beeinträchtigungen, Haft- und Vorstrafen, Alter, Faulheit) erschweren - vor allem für Jugendliche ohne Migrationshintergrund bzw. Burschen - die Arbeitssuche.

Einige Jugendliche haben den Eindruck, dass sich der Bewerbungsprozess auf Grund des unzureichenden Stellenangebots, der hohen Konkurrenz an MitbewerberInnen sowie ihrer mangelnden beruflichen Praxis für sie schwieriger gestaltet. Etwa ein Zehntel der Jugendlichen hat - ohne genaue Gründe angeben zu können - das Gefühl, dass andere bevorzugt werden bzw. am Arbeitsmarkt bessere Chancen haben als sie selbst. Das unbestimmte Gefühl der Benachteiligung ist bei österreichischen Jugendlichen und Mädchen etwas stärker ausgeprägt. 


\section{Die AMS-Beratung}

\subsection{Herausforderungen im Beratungsprozess}

Da rd. zwei Drittel aller jugendlichen AMS-KundInnen Jugendliche mit Migrationshintergrund sind, zählt die Beratung dieser KundInnen zum Berufsalltag der AMS-BeraterInnen. In vielen Bereichen decken sich die Probleme (z. B. schlechtes Ausbildungsniveau) und Bedürfnisse mit jenen der österreichischen AMS-KundInnen. Dennoch werden spezielle Herausforderungen im Umgang mit der Zielgruppe geortet.

Die sprachlichen Fähigkeiten der KundInnen mit Migrationshintergrund variieren sehr stark und reichen von überhaupt keinen Deutsch-Kenntnissen bis zu minimalen Defiziten der deutschen Sprache vor allem im schriftlichen Bereich. Die AMS-BeraterInnen beobachten, dass die Sprachkenntnisse nicht unbedingt mit der Aufenthaltsdauer in Österreich korrespondieren, da einige Jugendliche bereits in kurzer Zeit relativ gut die deutsche Sprache beherrschen, während andere, die bereits jahrelang in Österreich sind und oftmals hier auch die (gesamte) Schullaufbahn absolviert und positiv abgeschlossen haben, noch immer sprachliche Probleme haben. Die sprachlichen Fähigkeiten variieren in Abhängigkeit von der Lernfähigkeit und dem Bildungsniveau des/r einzelnen Kundln sowie der Häufigkeit der praktischen Anwendung der deutschen Sprache. Wenn Jugendliche sich weiterhin ausschließlich in der Muttersprache unterhalten, fernsehen und lesen, ist es kaum möglich, fließend Deutsch zu lernen. Diese sprachlichen Barrieren können zu Verständigungsproblemen sowie Missverständnissen im Beratungsgespräch führen und erfordern eine längere Beratungszeit, da die BeraterInnen das Gesagte wiederholen, umschreiben und erklären müssen. Wenn KundInnen massive Sprachprobleme aufweisen, kommen sie meist in Begleitung eines Dolmetschs oder die BeraterInnen wenden sich an andere KundInnen, die bei der Übersetzung helfen. Die MitarbeiterInnen mit Migrationshintergrund können von anderen BeraterInnen kaum - insbesondere nicht in Zonen mit exakter Kundeneinteilung (Service- und Beratungszone) - für Übersetzungshilfen herangezogen werden, da diese selbst eigene KundInnen zu betreuen haben, während dies in der Infozone etwas flexibler gehandhabt werden kann. Aber selbst die BeraterInnen mit Migrationshintergrund wenden nur äußerst selten im Rahmen der Kundengespräche ihre Sprachkenntnisse an, da eine Verständigung auf Deutsch mit den Jugendlichen gewünscht und auch meist möglich ist. Die weitere Vermittlung gestaltet sich bei sprachlichen Defiziten jedoch schwierig, da die DienstgeberInnen meist sehr gute Deutschkenntnisse voraussetzen.

Die AMS-MitarbeiterInnen sind auch mit verschiedenen kulturellen Traditionen der KundInnen konfrontiert, die die Vermittelbarkeit am Arbeitsmarkt einschränken. Mädchen, die ein Kopftuch tragen, sind nur sehr schwierig zu vermitteln, insbesondere in Berufen mit KundInnenkontakt, wie z. B. im Verkauf oder Friseurbereich. Die Bereitschaft, das Kopftuch abzunehmen, wird von den Beraterlnnen eher gering eingeschätzt. Wenn die Mädchen immer mit Kopftuch bei Vorstellungsgesprächen erscheinen und auf ihren Bewerbungsunterlagen mit Kopftuch abgebildet sind, reduziert dies ihre Chancen auf einen Arbeitsplatz. Ebenso schränken religiöse Vorschriften (z. B. im Umgang mit Schweinefleisch und Alkohol) die Vermittelbarkeit der Zielgruppe in gewissen Branchen (wie z. B. dem Gastgewerbe) ein. Die Alternative, auf Stellen bei UnternehmerInnen mit Migrationshintergrund auszuweichen, wird kaum angenommen, wie eine Beraterin erläutert: „Wenn man schaut in Geschäften, wo es ok wäre, von eigenen Landsleuten, das wollen die nicht. Das wollen sie bewusst nicht, weil sie sagen, da arbeiten wir für zu wenig Geld, die beuten uns aus. "Insbesondere türkische 
Mädchen weigern sich bei UnternehmerInnen aus ihrem Herkunftsland einen Job anzunehmen. Daher können die AMS-BeraterInnen oftmals nur auf die Eigeninitiative der KundInnen hoffen, dass sie sich verstärkt selbst um eine Stelle bemühen, wo diese kulturellen Symbole und religiösen Vorschriften akzeptiert werden.

Aber nicht nur kulturelle Unterschiede sondern auch allgemeine Vorurteile einheimischer UnternehmerInnen gegenüber Jugendlichen mit Migrationshintergrund erschweren die Stellensuche. Die MigrantInnen müssen höhere Qualifikationen mitbringen, um die gleichen Chancen auf einen Job zu haben wie Jugendliche aus Österreich. Oftmals schreckt allein auch ein „ausländisch“ klingender Name die potenziellen DienstgeberInnen bereits ab.

Des Weiteren beobachten die AMS-BeraterInnen Mentalitätsunterschiede, wobei KundInnen aus gewissen Herkunftsländern manchmal als sehr fordernd und verlangend erlebt werden. Der Anpassungswille wird bei verschiedenen Ethnien unterschiedlich wahrgenommen. Auch mit der Pünktlichkeit haben Jugendliche aus anderen Kulturkreisen oftmals mehr Probleme.

Ein weiteres bedeutendes Vermittlungshemmnis dieser Kundengruppe ist, dass ihr oftmals ein Pflichtabschluss fehlt bzw. dieser nicht anerkannt wird. Jugendliche, die ihren Pflichtabschulabschluss nicht in Österreich absolviert haben, werden beim AMS grundsätzlich als „ohne Pflichtschulabschluss“ erfasst. Dadurch ist lediglich eine Vermittlung in niedrig qualifizierte Stellen möglich, da eine Lehrausbildung einen positiven Pflichtschulabschluss voraussetzt. Somit müssen die betroffenen Jugendlichen zuerst einen Hauptschulabschlusskurs besuchen, bevor eine weitere Qualifizierung möglich ist, wobei jedoch das Angebot an diesen Basiskursen oft nicht ausreichend ist. Selbst wenn die KundInnen einen positiven Hauptschulabschluss vorweisen können, zeigen sich teilweise grobe Leistungsdefizite. Für die Beraterlnnen ist es oft schwierig, bei den festgestellten Defiziten konkrete Lösungs- und Weiterentwicklungsmöglichkeiten für die Jugendlichen zu entwickeln.

Außerdem nimmt die Familie und das soziale Umfeld teilweise stark auf die Kundlnnen mit Migrationshintergrund Einfluss. Dies kann einerseits direkt im Beratungsgespräch erfolgen, wenn sie als Begleitpersonen das Gespräch lenken und für den/die Kundln sprechen oder auch im Hintergrund stattfinden, indem sie geplante Aktivitäten infrage stellen oder nicht erlauben. Ein Beispiel dafür ist, dass ein Vater seiner Tochter verbietet, eine weiter entfernte (Lehr-) Stelle oder einen Kursplatz anzunehmen, weil er nicht will, dass sie so lange alleine unterwegs ist. Die Jugendlichen sind dann meist gezwungen, sich den elterlichen Vorstellungen zu fügen. Im Gegensatz dazu fehlt vielen KundInnen die familiäre Unterstützung gänzlich, die Eltern zeigen oftmals keinerlei Interesse an der schulischen und beruflichen Ausbildung ihrer Kinder. Sobald ihre Kinder in die Schule kommen, gehen einige Eltern davon aus, dass sich nun die LehrerInnen um ihre Kinder kümmern und daher keine weitere Einflussnahme notwendig ist. Die Eltern haben häufig auch keine realistischen Vorstellungen von den beruflichen Möglichkeiten am Arbeitsmarkt. Sie fokussieren weiterhin auf traditionelle Berufsbereiche und halten die beruflichen Erwartungen auch in Hinblick auf die geplante Zukunft (z. B. baldige Heirat der Töchter) teilweise bewusst niedrig. Auch die Erfahrungen der Eltern mit dem AMS können sich hinderlich auf die Beratungsgespräche mit den Jugendlichen auswirken. Wenn zu Hause über das AMS schlecht gesprochen wird, treten die jungen NeukundInnen den BeraterInnen bereits mit Vorbehalten entgegen. Die AMS-BeraterInnen würden sich daher eine produktive Zusammenarbeit mit den Eltern ihrer KundInnen wünschen, um gemeinsam leichter einen positiven Einfluss auf die Jugendlichen und ihren Werdegang ausüben zu können. 
Weitere Herausforderungen im Beratungsprozess betreffen sowohl Jugendliche mit Migrationshintergrund als auch österreichische KundInnen. Zahlreiche Jugendliche suchen das AMS ohne jegliche berufliche Perspektive auf und haben selbst (noch) keinerlei Idee, welchen beruflichen Weg sie einschlagen möchten. Die KundInnen haben häufig weder ein realistisches Bild von der Situation am Arbeitsmarkt noch von den Zielsetzungen des AMS. Sie glauben, dass sie wie ihre Eltern ohne Probleme eine Arbeit finden, weil sie jung und kräftig sind, auch wenn sie z. B. keinen Pflichtschulabschluss vorweisen können. Bei anderen wiederum besteht der Eindruck, dass sie das AMS nicht in erster Linie wegen der Vermittlung auf den Arbeitsmarkt aufsuchen, sondern, dass Ausbildungswünsche im Vordergrund stehen und sie eine Qualifizierungsmaßnahme nach der anderen besuchen wollen. Somit müssen die BeraterInnen oftmals Aufklärungsarbeit bezüglich Rahmenbedingungen und Zielvorgaben leisten.

Auch Motivationsmängel und Brüche in der Betreuung erschweren eine kontinuierliche Arbeit mit den Jugendlichen (mit und ohne migrantischem Hintergrund), da sie - insbesondere wenn sie Nicht-LeistungsbezieherInnen sind - nicht regelmäßig ihre AMSTermine einhalten. Für die BeraterInnen ist es dann schwierig, fortlaufende Maßnahmen für diese KundInnen zu setzen.

In den Beratungsgesprächen macht sich jedoch nicht nur der persönliche, familiäre und soziale Hintergrund der KundInnen, sondern auch jener der BeraterInnen bemerkbar. Die AMS-MitarbeiterInnen sind durch ihre eigene Sozialisation geprägt und können sich nicht immer in die Lebenswelt ihrer KundInnen hineinversetzen. Außerdem können auf Grund der langjährigen Tätigkeit beim AMS Vorurteile gegenüber den KundInnen entstehen. Um dies zu unterbinden, versuchen sich die MitarbeiterInnen dies bewusst zu machen, um zwischen Vorurteilen und tatsächlichen Voraussetzungen bei der/m jeweiligen Kunden/in zu unterscheiden. 


\subsection{Die AMS-Besuche der Jugendlichen}

$38 \%$ der befragten jugendlichen AMS-KundInnen sind erst seit maximal 2 Monaten mit dem Wiener AMS Jugendliche in Kontakt, wie Grafik 37 zeigt. $15 \%$ sind 3 bis 5 Monate beim AMS vorgemerkt und ein Viertel gibt eine Arbeitslosigkeitsdauer zwischen einem halben Jahr und einem Jahr an. Ein weiteres Viertel ist bereits länger als 12 Monate arbeitslos. MigrantInnen sind tendenziell länger arbeitslos als Jugendliche ohne Migrationshintergrund, denn sie sind in der Gruppe der AMS-Neuzugänge mit einer Vormerkdauer von 0 bis 2 Monaten deutlich weniger vertreten als österreichische Jugendliche (36\% vs. $44 \%$ ) und häufiger mit einer Arbeitslosigkeitsdauer von länger als einem Jahr konfrontiert (26\% vs. $18 \%$ ).

Grafik 37 Vormerkdauer beim AMS nach Migrationshintergrund, Anteil der befragten Jugendlichen nach Migrationshintergrund in Prozent

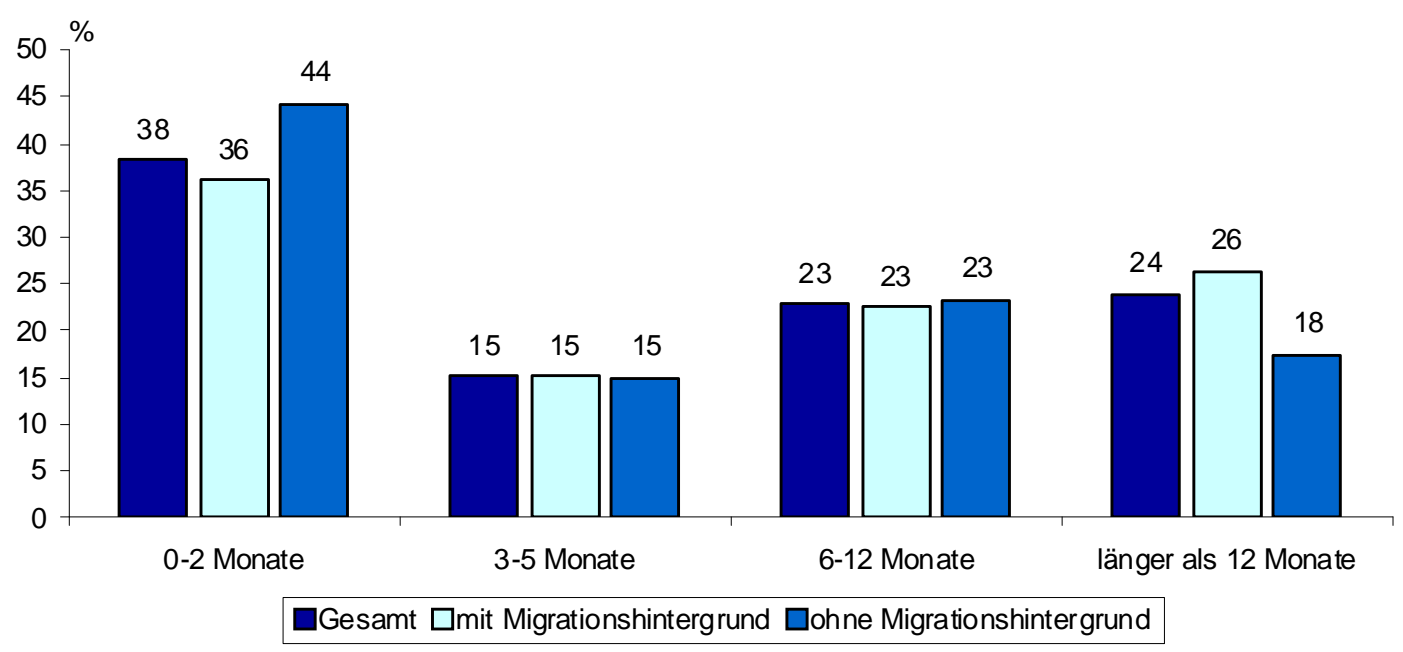

Quelle: KMU FORSCHUNG AUSTRIA, Befragung der jugendlichen AMS-KundInnen, $n=431$

Mehr als ein Drittel der befragten Jugendlichen (35\%) wendet sich aus Eigeninitiative an das AMS, dies trifft insbesondere auf KundInnen ohne Migrationshintergrund (46\%) zu (siehe Grafik 38). Für ein weiteres Drittel (34\%) waren die Eltern bzw. die Familie die Impulsgeber für ihren ersten AMS-Besuch. Bei österreichischen Jugendlichen gab die Familie stärker den Anstoß, das AMS aufzusuchen, als bei MigrantInnen (39 \% vs. $32 \%)$. Dennoch haben die Eltern bzw. die Familie für die Jugendlichen mit Migrationshintergrund im Vergleich zur Erhebung im Jahr 2007 als Hinweisgeber für den AMSBesuch an Bedeutung gewonnen (32 \% vs. $25 \%$ ) (vgl. Heckl et al., 2007). Ein knappes Fünftel (19\%) wandte sich auf Grund von Informationen aus der Schule und rd. $13 \%$ dank Hinweisen von FreundInnen und Bekannten an das AMS. Vor allem für Personen mit Migrationshintergrund waren die Informationen aus der Schule, aber auch jene von FreundInnen und Bekannten, wesentlich, damit sie mit dem AMS Kontakt aufnehmen. Weitere InformantInnen, die den Jugendlichen nahe gelegt haben, sich beim AMS zu melden, sind ehemalige ArbeitgeberInnen, MitarbeiterInnen bei Kursen/Schulungen, diverse Ämter (wie z. B. Finanzamt, Jugendamt) sowie verschiedene Beratungsstellen. 
Von Seiten der Schulen wird versucht, im Rahmen von Besuchen des AMS auf dieses Serviceangebot im Falle von Arbeitslosigkeit oder zur Unterstützung bei der Lehrstellensuche aufmerksam zu machen. Rd. $35 \%$ der befragten Jugendlichen haben bereits einmal mit der Schule das AMS besucht. Personen mit Migrationshintergrund waren etwas häufiger mit der Schule beim AMS als Jugendliche ohne Migrationshintergrund (37\% vs. $31 \%)$.

Grafik 38 Impulsgeber für den ersten AMS-Besuch nach Migrationshintergrund, Anteil der befragten Jugendlichen nach Migrationshintergrund in Prozent

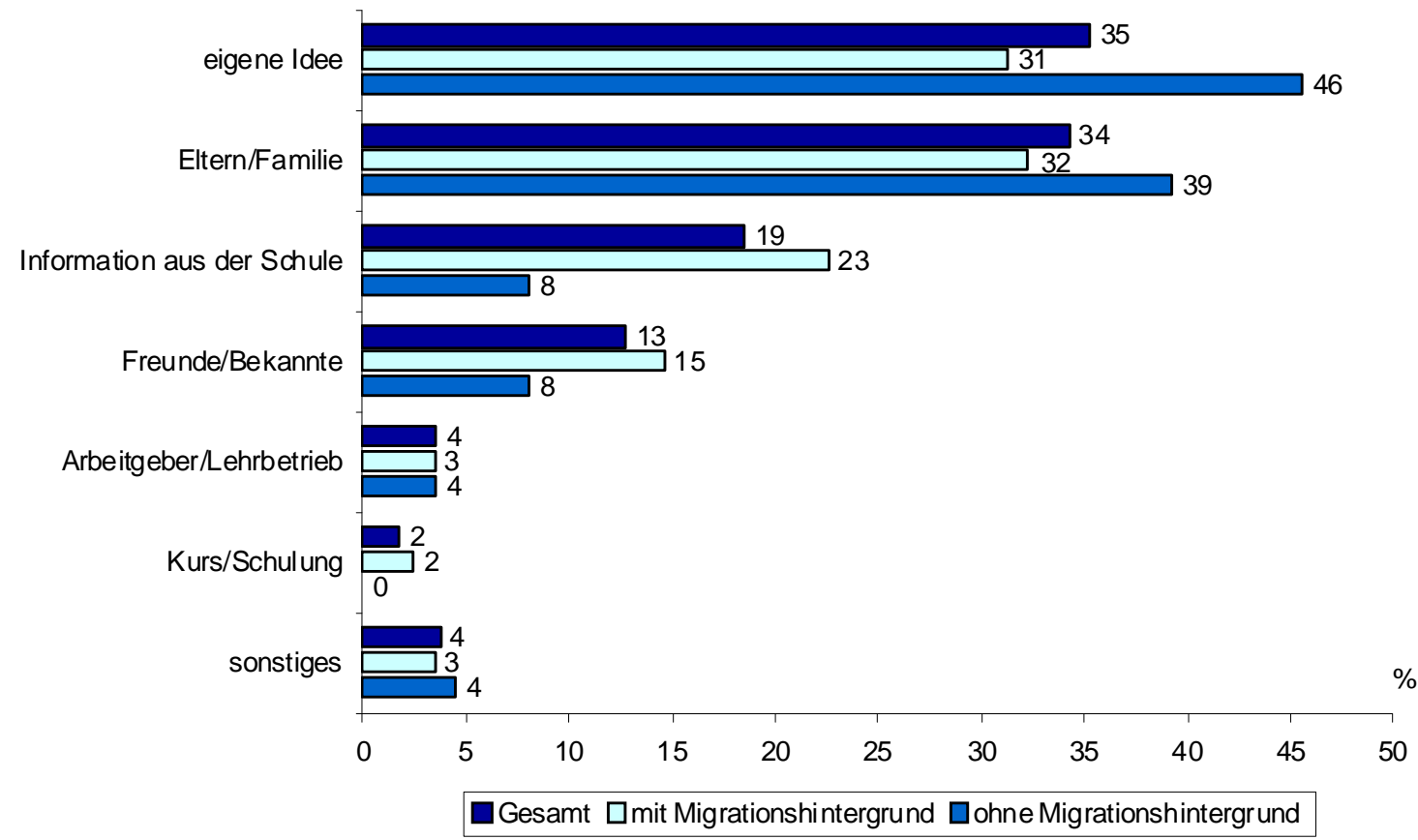

Quelle: KMU FORSCHUNG AUSTRIA, Befragung der jugendlichen AMS-KundInnen, $n=400$, Mehrfachnennungen möglich

Die Mehrheit der befragten jugendlichen AMS-KundInnen kommt in erster Linie wegen der Suche nach einer Arbeit oder einer Lehrstelle zum AMS (siehe Grafik 39). Mehr als die Hälfte der Jugendlichen (53\%) ist an einer Arbeitsvermittlung interessiert, wobei dies vor allem auf die älteren Jugendlichen zutrifft. Rd. $62 \%$ der 19-Jährigen und $79 \%$ der Über-20-Jährigen suchen in erster Linie eine Arbeit. Bei den jüngeren Jugendlichen steht hingegen die Lehrstellensuche im Vordergrund: $71 \%$ der Unter-16-Jährigen hoffen vom AMS in eine Lehrstelle vermittelt zu werden (ein Drittel aller Jugendlichen).

Etwa ein Zehntel der Jugendlichen (11\%) ist an einer Qualifizierungsmaßnahme vom AMS interessiert. Insbesondere Mädchen (15\%) und MigrantInnen (14\%) streben einen Kurs- oder Schulungsbesuch an und erhoffen sich diesbezüglich Unterstützung vom AMS.

$8 \%$ der Befragten erwarten sich vom AMS finanzielle Unterstützung, dies trifft vor allem auf österreichische Jugendliche (13\%) und KundInnen ab 18 Jahren zu, da diese eher schon Arbeitserfahrung gesammelt und daher auch Ansprüche auf finanzielle Leistungen erworben haben. 
Weitere Gründe für die Jugendlichen, das AMS aufzusuchen, sind Hilfestellungen bei der beruflichen Orientierung, der erforderliche Nachweis von Bestätigungen beim Finanzamt etc., die Weiterversicherung, Kontrolltermine oder die Meldung nach Kursund Schulbesuchen bzw. nach einer Kündigung.

Grafik 39 Gründe für den AMS-Besuch, Anteil der befragten Jugendlichen nach Migrationshintergrund in Prozent

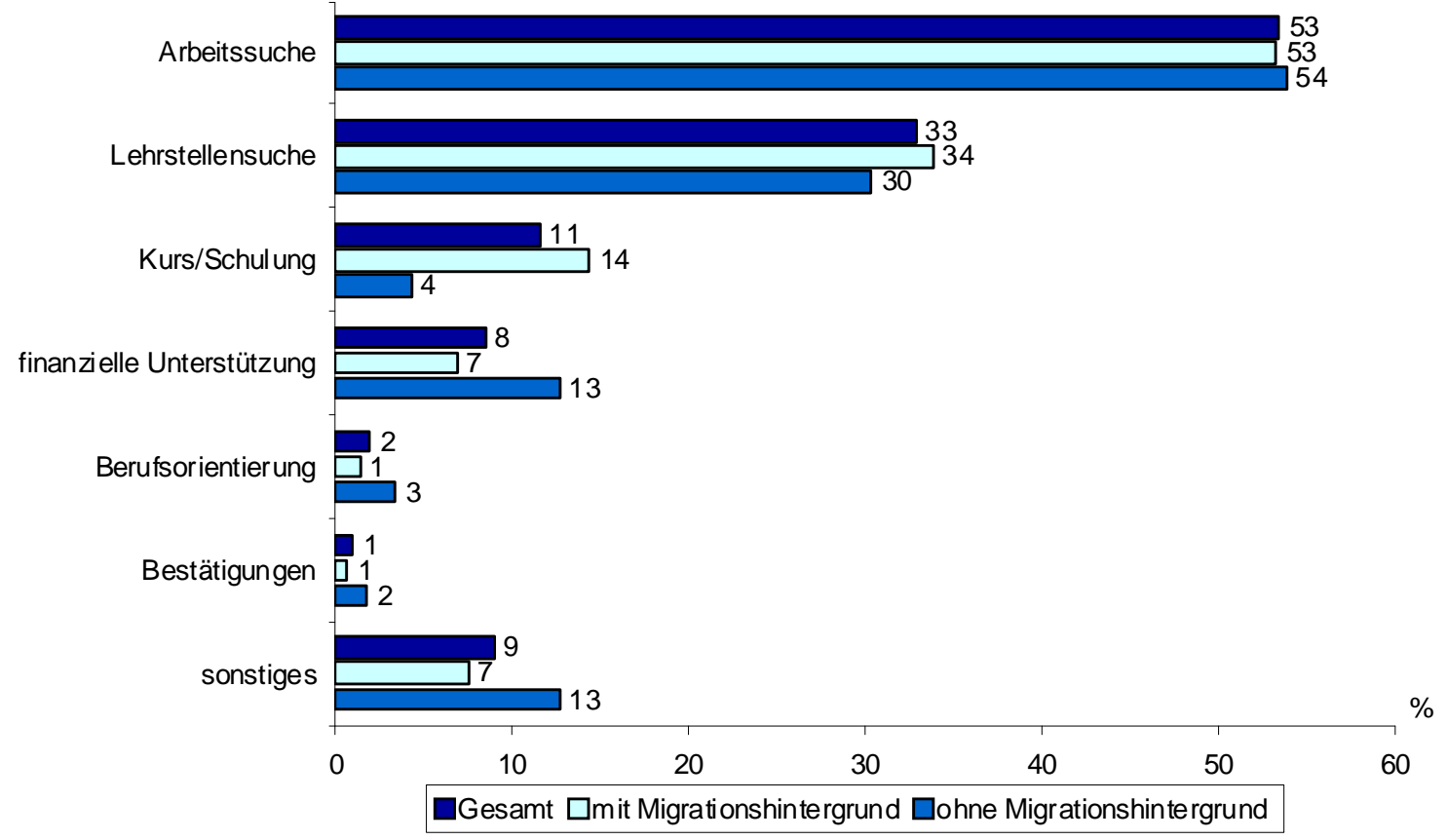

Quelle: KMU FORSCHUNG AUSTRIA, Befragung der jugendlichen AMS-KundInnen, $n=427$, Mehrfachnennungen möglich

\subsection{Bewertungen der AMS-Leistungen}

\subsubsection{Beratungsgespräche}

$71 \%$ der befragten Jugendlichen hatten bereits öfter Beratungsgespräche mit ihrem/r Beraterln. $18 \%$ der Befragten waren zum Zeitpunkt der Befragung erst einmal mit ihrem/r Beraterln in Kontakt und rd. ein Zehntel (11\%) wurde bisher noch nicht beraten.

Mehr als $60 \%$ der Befragten (mit und ohne Migrationshintergrund), die bisher mindestens ein Beratungsgespräch führten, hat das letzte Gespräch sehr oder etwas geholfen, wie Grafik 40 zeigt. Damit war das letzte Beratungsgespräch für die jugendlichen MigrantInnen genauso hilfreich wie bei der Erhebung im Jahr 2007 (vgl. Heckl et al., 2007). Die Beratung war für die KundInnen vor allem dann eine Hilfe, wenn ein Kursbesuch vereinbart oder bewilligt wurde. Außerdem haben sie es als hilfreich empfunden, dass innen Stellenanzeigen für freie Arbeitsplätze oder Lehrstellen mitgegeben wurden. 
Weitere Hilfe haben die Jugendlichen erfahren, indem sie relevante Informationen von ihren BeraterInnen bekamen und beim Bewerbungsprozess und der Berufswahl unterstützt wurden. Bei den Beratungsgesprächen werden vor allem ein freundliche und nette Gesprächsbasis, gute und ausführliche Erklärungen, Motivationsarbeit und unterstützende Hilfeleistungen von den jugendlichen KundInnen sehr geschätzt.

Für beinahe $40 \%$ der AMS-KundInnen (mit und ohne Migrationshintergrund) war jedoch - ebenso wie im Jahr 2007 - das letzte Gespräch mit ihrem/r Beraterln weniger oder überhaupt nicht hilfreich (vgl. Heckl et al., 2007). Dies war in erster Linie dann der Fall, wenn keine geeigneten freien Arbeits- oder Lehrstellen gefunden werden konnten. Mit der selbstständigen Suche fühlen sich manche Jugendliche überfordert, wie folgendermaßen geschildert wird: „Ich bin auf mich selbst gestellt, bekomme keine Stellen angeboten, muss aber Bewerbungen bringen, sehe den Sinn nicht darin." Wenig hilfreich ist es auch für die Jugendlichen, wenn die gewünschte Qualifizierung nicht genehmigt wurde oder ein Kursbesuch vorgeschlagen wird, der nicht ihren Vorstellungen entspricht, wie durch folgende Aussagen deutlich wird: „Eventuell bekomme ich eine Schulung hat es geheißen, dann habe ich aber doch nichts bekommen." bzw. "Man wird nur in Kurse geschickt, man wird aber nicht gefragt, was man will." Darüber hinaus wird als negativ empfunden, wenn die KundInnen nichts Neues erfahren, nur allgemeine Informationen erhalten und die BeraterInnen kein Interesse zeigen bzw. zu wenig auf die Wünsche der Jugendlichen eingehen. Auch wenn beim Beratungsgespräch vorwiegend administrative Angelegenheiten und Formalitäten abgewickelt bzw. nur weitere Termine vereinbart werden, erscheint dies den KundInnen wenig hilfreich.

Grafik 40 Bewertung des letzten Beratungsgesprächs, Anteil der befragten Jugendlichen, die mindestens ein Beratungsgespräch hatten, nach Migrationshintergrund in Prozent

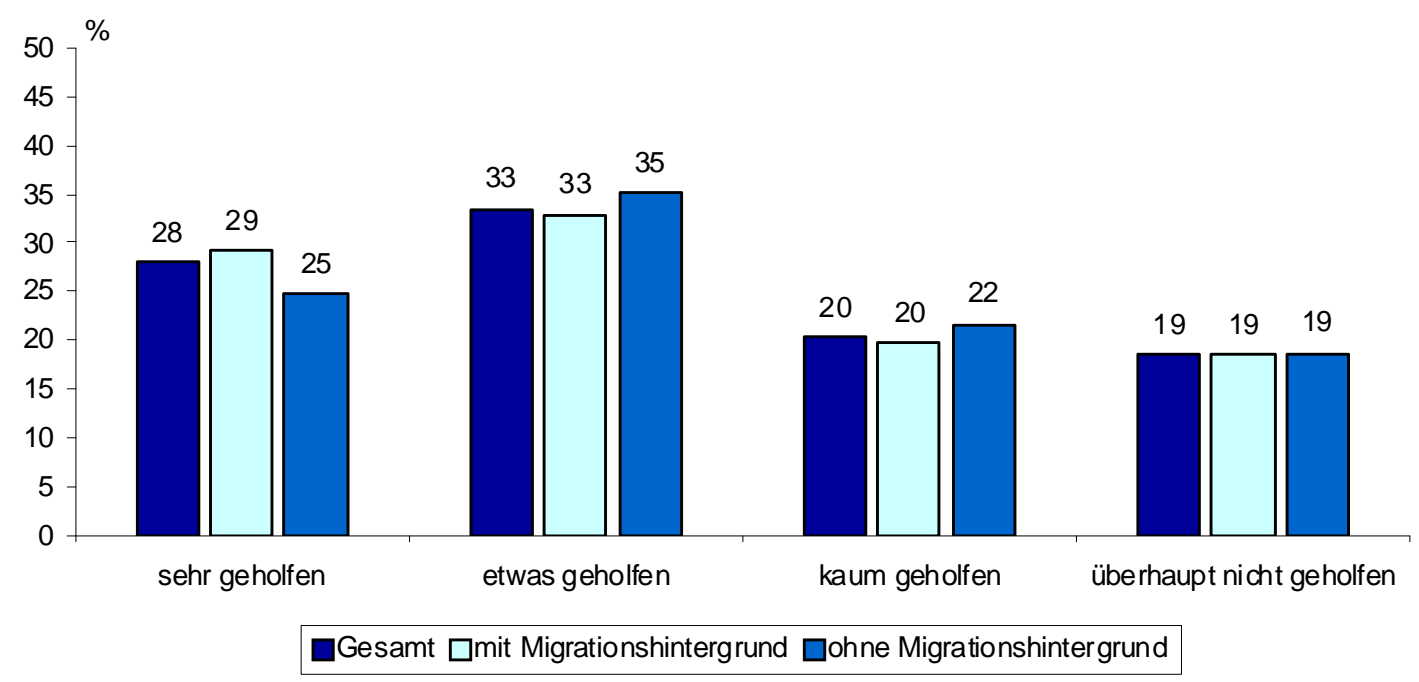

Quelle: KMU FORSCHUNG AUSTRIA, Befragung der jugendlichen AMS-KundInnen, $\mathrm{n}=362$

Insgesamt sind $83 \%$ der befragten AMS-KundInnen mit ihrem/r Beraterln sehr bzw. eher zufrieden, wie aus Grafik 41 ersichtlich. Insbesondere bei den österreichischen Jugendlichen ist der Anteil der "sehr Zufriedenen“ höher als bei den MigrantInnen (60\% vs. $49 \%)$. 
Auch wenn sich das Ausmaß der "sehr Zufriedenen“ von Seiten der KundInnen mit Migrationshintergrund im Vergleich zur Erhebung im Jahr 2007 etwas reduziert hat (von $54 \%$ auf $49 \%$ ), ist die Zufriedenheit mit den Beraterlnnen insgesamt mit $83 \%$ genauso hoch wie im Jahr 2007 (vgl. Heckl et al., 2007).

Die AMS-KundInnen sind mit ihren BeraterInnen zufrieden, wenn diese nett, freundlich und höflich zu innen sind und innen ausführliche, verständliche Auskünfte erteilen. Sie schätzen auch, dass die AMS-BeraterInnen auf ihre Berufswünsche und Probleme eingehen und sie bei der (Lehr-)Stellensuche motivieren. Den Jugendlichen gefällt, dass sie sich um sie kümmern, gut beraten und ihnen hilfreiche Informationen und Erklärungen geben. Einzelne KundInnen schätzen auch die rasche, lockere Abwicklung der Gespräche.

Lediglich $17 \%$ der Jugendlichen sind mit ihrem/r Beraterln weniger oder überhaupt nicht zufrieden. Diese beschreiben die für sie zuständigen AMS-MitarbeiterInnen als unfreundlich, distanziert oder streng und haben das Gefühl von ihnen nicht verstanden bzw. nicht ernst genommen zu werden. Die Jugendlichen haben teilweise den Eindruck, dass die Beraterlnnen nicht auf sie eingehen, fühlen sich alleine gelassen und bei der Stellensuche von innen unter Druck gesetzt. Weitere genannte Kritikpunkte betreffen die kurze Beratungszeit, unzureichende Auskünfte sowie häufige Beraterwechsel.

\section{Grafik $41 \quad$ Zufriedenheit mit den AMS-BeraterInnen, Anteil der befragten Jugendlichen, die mindestens ein Beratungsgespräch hatten, nach Migrationshintergrund in Prozent}

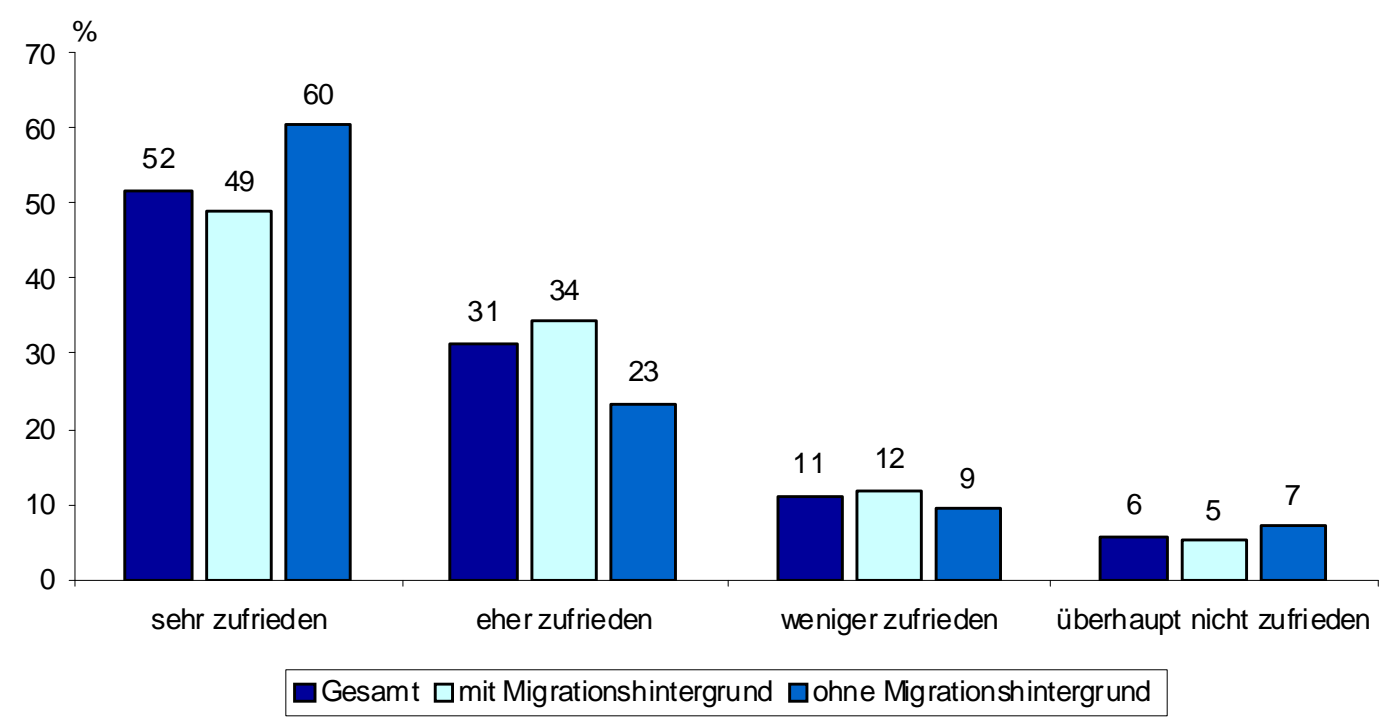

Quelle: KMU FORSCHUNG AUSTRIA, Befragung der jugendlichen AMS-KundInnen, $\mathrm{n}=340$

Auch die BeraterInnen erleben die Viertelstunde, die in der Regel für die Beratungsgespräche zur Verfügung steht, oftmals als relativ kurz, da sie zahlreiche Formalitäten im Rahmen der Beratungsgespräche erledigen müssen. Um diesen zeitlichen Engpässen zu entkommen, können die Beraterlnnen drei Mal pro Woche längere Beratungsgespräche von einer halben Stunde vereinbaren. Für diese aufwändigeren Gespräche mit schwierigen Fällen sind aber oft auch 30 Minuten zu wenig. Eine weitere Alternative ist, die KundInnen bei zusätzlichem Beratungsbedarf zu externen Beratungseinrichtungen, wie z. B. C'mon 14/17 oder an das Beratungszentrum für 
MigrantInnen, zu verweisen. Dort können sich die Jugendlichen an Personen wenden, die ausreichend Zeit haben, um tieferliegende - zum Teil auch private - Probleme zu bearbeiten. Diese umfassende Unterstützung können die AMS-BeraterInnen im Rahmen ihrer Beratungsgespräche nicht bieten, daher werden diese externen Angebote für die Zielgruppe als sehr wertvoll erlebt.

\subsubsection{Stellenanzeigen}

Eine Aufgabe der AMS-BeraterInnen ist es, den Kundlnnen regelmäßig Stellenanzeigen mitzugeben bzw. innen per E-Mail oder Post zu schicken. $65 \%$ der befragten Jugendlichen haben vom AMS schon Stellen zugeschickt oder ausgedruckt bekommen. Bei einem Drittel dieser KundInnen stimmten die Stellen sehr mit ihren Berufswünschen überein (siehe Grafik 42). Für $38 \%$ der MigrantInnen und $41 \%$ der österreichischen Jugendlichen entsprachen die Inserate nur teilweise ihren beruflichen Vorstellungen. Insgesamt war mehr als ein Viertel (28\%) der Auffassung, dass die Stellenanzeigen überhaupt nicht mit ihren Berufswünschen korrespondierten, dies traf auf Jugendliche mit Migrationshintergrund etwas häufiger zu als auf österreichische KundInnen (30 \% vs. $25 \%$ ). Generell hat sich das Ausmaß der Übereinstimmung der Stellenanzeigen mit den Berufswünschen der jugendlichen MigrantInnen zwischen 2007 und 2010 kaum verändert (vgl. Heckl et al., 2007).

Jene KundInnen, die sich über eine mangelnde Übereinstimmung beklagten, haben in erster Linie Anzeigen erhalten, die nicht der gewünschten Branche bzw. den angestrebten Berufen entsprachen. Teilweise bestand sogar der Eindruck, dass sie Stellen in Berufsbereichen bekamen, die sie vorher ausdrücklich ausgeschlossen hatten. In Einzelfällen trugen auch Unwissenheit und sich ändernde berufliche Vorstellungen zu diesen Unstimmigkeiten bei, wie ein Jugendlicher berichtet: „Das erste Mal wollte die Beraterin wissen, welchen Beruf ich machen will. Da ich es nicht wusste, habe ich einfach Security gesagt. Ich will das aber nicht machen. Seitdem gibt sie mir immer Stellen, die nicht so passen. "Die unzureichende Übereinstimmung kann aber auch den Umfang des Arbeitsverhältnisses (Teilzeit vs. Vollzeit), die mitzubringenden Voraussetzungen (erforderliche Schulabschlüsse, Berufserfahrung) und die Anforderungen bei dem neuen Job (Hilfstätigkeit vs. qualifizierte Arbeit) betreffen. Darüber hinaus beklagen die jugendlichen AMS-KundInnen, dass einige der übermittelten Stellenanzeigen nicht mehr aktuell und daher bereits vergeben waren, die Entfernung zwischen Wohn- und zukünftigem Arbeitsort zu weit war oder die Unternehmen (Leiharbeit, migrantische Unternehmen) nicht den persönlichen Vorstellungen entsprachen. Leasingfirmen sowie andere Personalvermittlungsunternehmen sind laut Auskunft der AMSMitarbeiterInnen bei den Jugendlichen generell nicht sehr beliebt. 
Grafik 42 Übereinstimmung der Stellenanzeigen des AMS mit den Berufswünschen der Jugendlichen, Anteil der befragten Jugendlichen, die Stellen erhalten haben, nach Migrationshintergrund in Prozent

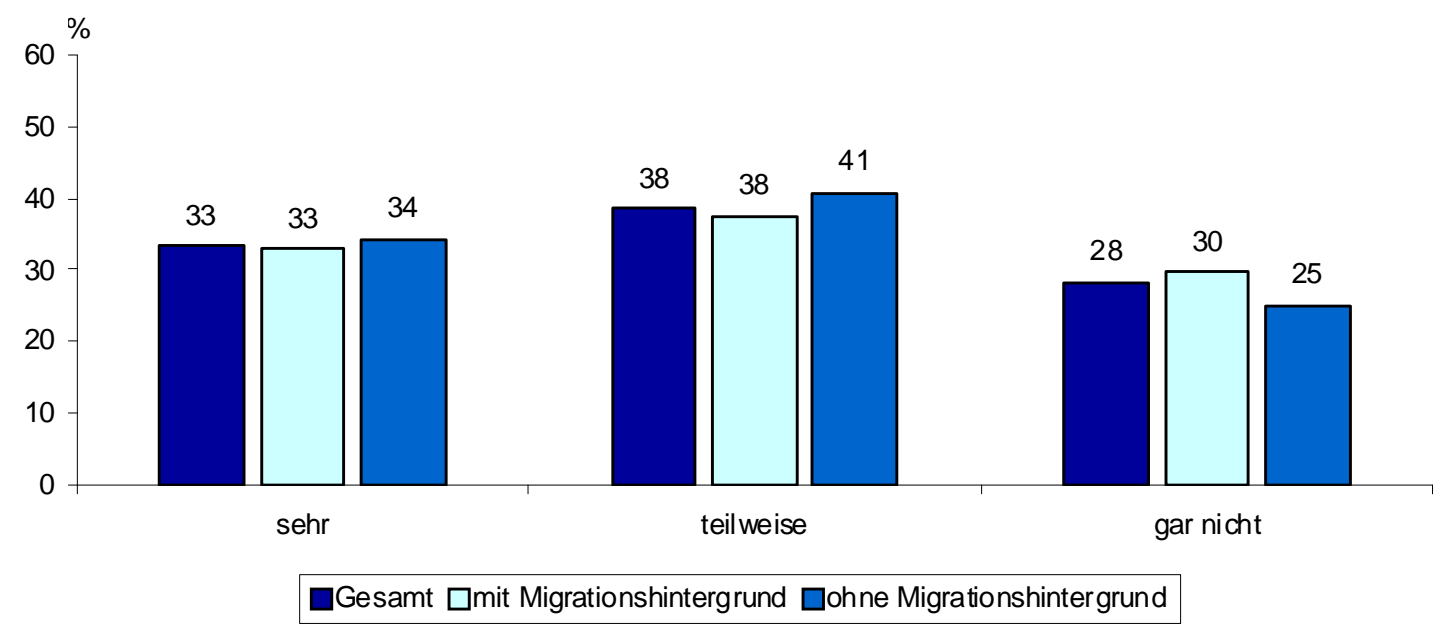

Quelle: KMU FORSCHUNG AUSTRIA, Befragung der jugendlichen AMS-KundInnen, n = 252

Aber auch das AMS hat aus Sicht der BeraterInnen bei den Jugendlichen keinen guten Ruf als Personalvermittler. Die AMS-MitarbeiterInnen haben den Eindruck, dass die KundInnen das Gefühl haben, dass die Unternehmen, die über das AMS suchen, wenig bezahlen und keine guten Dienstgeberlnnen sind. Daher bewerben sich manche überhaupt nicht bei den übermittelten Stellen. Das wirkt sich natürlich negativ auf den Vermittlungserfolg aus und ist für die BeraterInnen teilweise auch frustrierend.

\subsubsection{Kurse/Maßnahmen}

Auf Grund der niedrigen Qualifikation oder weil die Jugendlichen nicht unmittelbar „jobready" sind, werden sie oftmals auch in Weiterbildungsaktivitäten vermittelt. Insgesamt haben bereits $59 \%$ der befragten Jugendlichen an einem Kurs oder einer Maßnahme des AMS teilgenommen. Dabei waren mehr Kundlnnen mit Migrationshintergrund (62\%) sowie Jugendliche mit Arbeitserfahrung (61\%) in die Qualifizierungs- oder Aktivierungsaktivitäten des AMS eingebunden als österreichische Jugendliche (51 \%) bzw. KundInnen ohne berufliche Praxis (53\%). Das Ausmaß der Kursbesuche der Jugendlichen mit Migrationshintergrund ist ähnlich wie im Jahr 2007, wo $65 \%$ der Befragten an einem AMS-Kurs teilnahmen (vgl. Heckl et al., 2007). Einige Kundlnnen haben nicht nur einen Kurs besucht, sondern bereits eine lange „Kurskarriere“ hinter sich, wie ein Mädchen erzählt: „Ich habe schon sehr viele Kurse besucht. Ich habe erstens den BOCO-Kurs gemacht, nach dem BOCO-Kurs ging ich wieder in einen BOCO-Kurs, weil ich den ersten BOCO-Kurs unterbrochen habe. Dann habe ich so einen JASG-Kurs gemacht, das sind die 3 Jahre Lehre, die man machen muss, als Lagerlogistikerin, da war ich 2 Monate oder so. Dann habe ich im 20. [Bezirk] einen Kurs besucht, das, glaube ich, das war's. "Bei knapp einem Fünftel der KundInnen, die noch keinen Kurs absolviert haben, ist ein Kursbesuch geplant. Dies betrifft vor allem Jugendliche ohne berufliche Erfahrung und Mädchen.

Mehr als die Hälfte der befragten KundInnen (53\%), die bereits einen Kurs besuchten, nahm an Maßnahmen zur Berufsorientierung und Vorbereitung auf den Arbeitsmarkt teil, um mit dem Bewerbungsprozess vertraut zu werden und leichter in die Berufswelt einsteigen zu können (siehe Grafik 43). Männliche Jugendliche (60 \%) waren häufiger 
in diese vorbereitenden Kursaktivitäten eingebunden als die weibliche Zielgruppe (43\%). Etwa ein Viertel aller Befragten mit Kursbesuchen absolvierte umfassendere Lehr- und Berufsausbildungen, vor allem im Rahmen der JASG-Lehrgänge, aber auch durch die Teilnahme an berufsrelevanten Ausbildungen, wie z. B. zur KindergartenassistentIn, Ordinationshilfe etc. Insbesondere Jugendliche mit Migrationshintergrund nahmen öfter Lehr- und Berufsausbildungen des AMS in Anspruch als KundInnen ohne migrantischen Hintergrund ( $28 \%$ vs. $12 \%$ ), da sie es bei der Suche nach Lehrstellen und Ausbildungsplätzen am Arbeitsmarkt schwieriger haben als österreichische Jugendliche.

$14 \%$ der Jugendlichen erhielten eine Ausbildung im Büro- oder EDV-Bereich, um z. B. den Europäischen Computerführerschein (ECDL) zu erwerben oder sich kaufmännische Kenntnisse anzueignen. Vor allem KundInnen aus Österreich konnten sich in diesen Bereichen weiterqualifizieren, während dies auf MigrantInnen seltener zutraf (25\% vs. $11 \%)$.

$12 \%$ aller jugendlichen Kundlnnen bzw. $16 \%$ der Jugendlichen mit Migrationshintergrund besuchten einen Deutschkurs. Migrantinnen nahmen deutlich häufiger einen Deutschkurs in Anspruch als Migranten (23\% vs. $11 \%$ ) Damit fällt der Bedarf nach einer Qualifizierung in der deutschen Sprache bei der jungen Zielgruppe deutlich niedriger aus als bei erwachsenen KundInnen mit Migrationshintergrund, da in den Regionalen Geschäftsstellen Huttengasse und Laxenburger Straße in Wien jeweils mehr als ein Drittel der befragten erwachsenen MigrantInnen an Deutschkursen oder Alphabetisierungsmaßnahmen teilnahmen (vgl. Heckl et al., 2009).

Etwas mehr als ein Zehntel der Jugendlichen (11\%) erwarb mit Hilfe des AMS eine Zusatzqualifikation in Form einer technischen Ausbildung (z. B. Staplerschein) oder einer fremdsprachlichen Qualifizierung (vorwiegend in Englisch). Insbesondere KundInnen aus Österreich (25\%) waren TeilnehmerInnen an Zusatzausbildungen, während MigrantInnen (6\%) diese wesentlich seltener in Anspruch nahmen.

$7 \%$ der KundInnen wurden an Leiharbeitsfirmen, die die Jugendlichen verstärkt betreuen, verwiesen, um ihre Integration in den Arbeitsmarkt zu beschleunigen. $7 \%$ aller Jugendlichen bzw. 15 \% der Mädchen nahmen an speziellen Kursen für Mädchen teil, die sie bei der Berufsorientierung unterstützten und ihnen den Zugang zu technischen und handwerklichen Berufen eröffneten. Trotz dieses Kursangebots stößt der technische Berufsbereich laut Auskunft der AMS-Beraterlnnen bei der Zielgruppe noch weiterhin auf wenig Gegenliebe. Neben Maßnahmen, die den Zugang zu technischen Berufsfeldern fördern, werden auch spezielle Ausbildungen für Mädchen (wie z. B. Kindergartenhelferin, Call-Center Agent) angeboten, während für männliche Jugendliche kaum spezifische Kurse zur Verfügung stehen. Das wird von den BeraterInnen teilweise als Diskriminierung empfunden und benachteiligt die Burschen bei Qualifizierungswünschen in „untypischen“ Berufen.

Darüber hinaus besuchten $4 \%$ der Jugendlichen Kurse, um (nachträglich) den Hauptschulabschluss zu erwerben, und so den Anforderungen des Arbeits- und Ausbildungsmarktes besser gerecht zu werden. KundInnen mit Migrationshintergrund (und dabei vor allem Mädchen) nahmen die Gelegenheit, den Hauptschulabschluss im Rahmen eines AMS-Kurses zu absolvieren, etwas häufiger wahr als österreichische Jugendliche (5\% vs. $2 \%$ ). 
Grafik 43 Art der besuchten Kurse/Maßnahmen, Anteil der befragten Jugendlichen, die bereits an einem Kurs teilgenommen haben, in Prozent

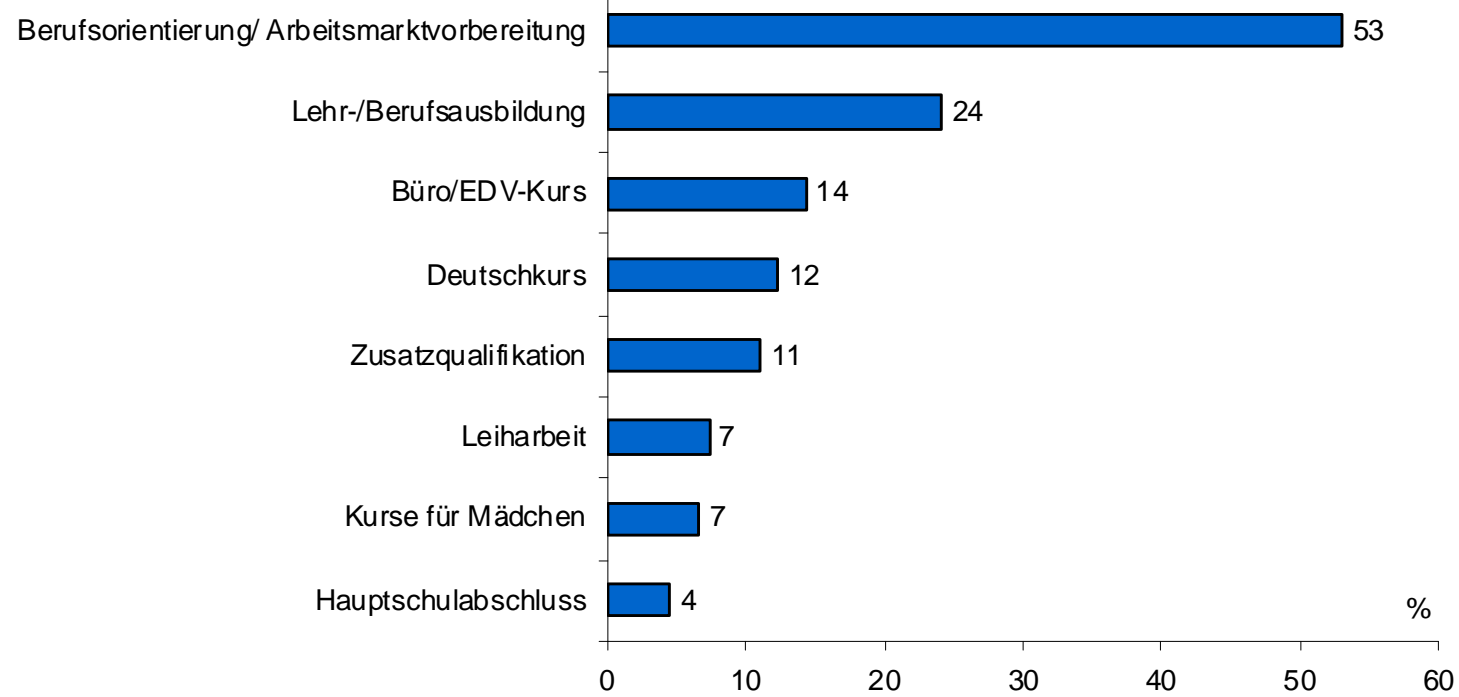

Quelle: KMU FORSCHUNG AUSTRIA, Befragung der jugendlichen AMS-KundInnen, n = 245, Mehrfachnennungen möglich

Die Kursbesuche kamen in etwas mehr als der Hälfte der Fälle (55\% bzw. $56 \%$ ) bei KundInnen mit und ohne Migrationshintergrund auf Vorschlag des/r AMS-Beraters/in zustande. Auch bei der Erhebung im Jahr 2007 fand mehr als die Hälfte der Kursbesuche (52\%) der MigrantInnen auf Vorschlag des/r Beraterln statt (vgl. Heckl et al., 2007). Ähnliche Erfahrungen haben die Jugendlichen, die im Rahmen der Fokusgruppen befragt wurden, gemacht: „Meine Beraterin hat mich hierher geschickt. Sie hat gesagt, dass ist eine Schule und ich habe gesagt, ok dass ist eh gut." $29 \%$ der Maßnahmenteilnahmen wurden aus eigenem Wunsch der jugendlichen KundInnen angeregt und $14 \%$ der Kursbesuche wurden gemeinsam von dem/r Beraterln und dem/r Jugendlichen erarbeitet.

Bei den verschiedenen Kursen sind jedoch erhebliche Unterschiede in Hinblick auf die Art des Zustandekommens zu beobachten, wie Grafik 44 zeigt. Der Besuch von Hauptschulabschlusskursen erfolgte vorwiegend auf eigenen Wunsch (64\%). Auch bei Ausbildungen im Büro- und EDV-Bereich sowie bei Deutschkursen spielte die Eigeninitiative eine bedeutende Rolle für Zuweisung zu den gewünschten Kursmaßnahmen. Hingegen kamen Berufsorientierungsmaßnahmen und Aktivitäten zur Vorbereitung auf den Arbeitsmarkt (71 \%) in erster Linie auf Vorschlag der BeraterInnen zustande. Auch Kurse für Mädchen, zusätzliche technische oder sprachliche Qualifizierungen sowie Vermittlungen in Leiharbeit wurden jeweils in etwa der Hälfte der Fälle auf Initiative des/r Berater/in realisiert. 


\section{Grafik $44 \quad$ Art des Zustandekommens der letzten zwei Kursbesuche, Anteil der} Kursbesuche in Prozent ${ }^{1}$

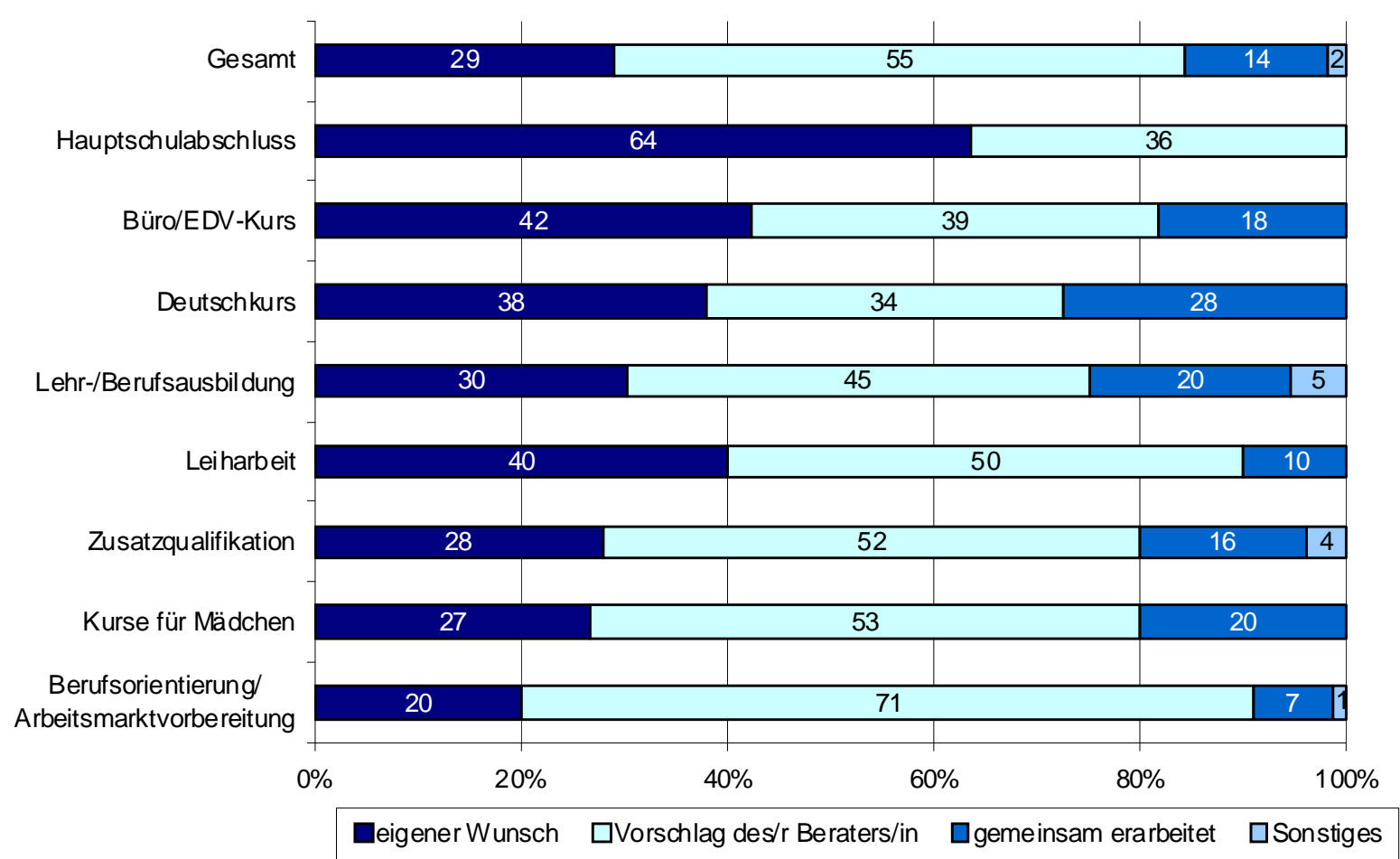

${ }^{1}$ Grundlage bilden jeweils die Anzahl der Kursbesuche (nach Kursart), da jede/r Kundln zu den letzten zwei Kursen detaillierte befragt wurde.

Quelle: KMU FORSCHUNG AUSTRIA, Befragung der jugendlichen AMS-KundInnen, $\mathrm{n}=313$ Kurse

Vor dem Kursbesuch werden die KundInnen von den AMS-BeraterInnen über Inhalt und Zweck des Kurses informiert. Insgesamt hatten die befragten Jugendlichen bei etwa zwei Drittel (65\%) der Maßnahmenteilnahmen den Eindruck, dass sie davor ausreichend Informationen vom AMS erhielten, wie aus Grafik 45 ersichtlich. KundInnen ohne Migrationshintergrund fühlten sich vor ihren Kursbesuchen noch etwas besser informiert als MigrantInnen (bei $69 \%$ vs. $63 \%$ der Kursteilnahmen). Vor allem vor Besuchen von mädchenspezifischen Maßnahmen, Hauptschulabschluss- und Deutschkursen erhielten die Jugendlichen ausreichend Informationen über die bevorstehende Qualifizierungsmaßnahme. Insbesondere MigrantInnen fühlten sich vor dem Besuch einer Zusatzqualifizierung (ebenso wie vor der Teilnahme an Berufsorientierungsmaßnahmen) nicht so gut informiert wie österreichische Jugendliche. 


\section{Grafik $45 \quad$ Ausreichend Informationen vor dem Kursbesuch erhalten, Anteil der} Kursbesuche in Prozent ${ }^{1}$

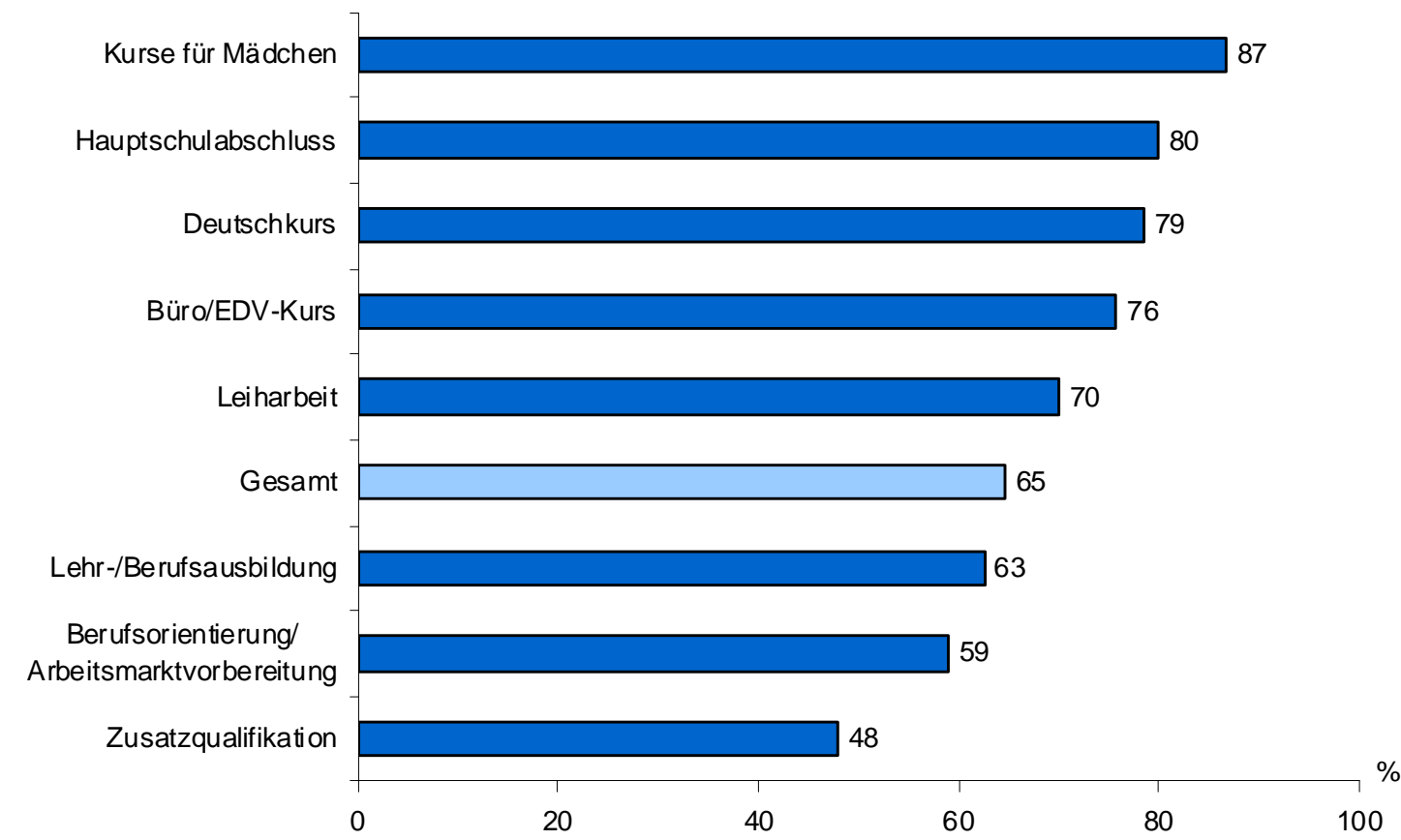

${ }^{1}$ Grundlage bilden jeweils die Anzahl der Kursbesuche (nach Kursart), da jede/r Kundln zu den letzten zwei Kursen detaillierte befragt wurde.

Quelle: KMU FORSCHUNG AUSTRIA, Befragung der jugendlichen AMS-KundInnen, $\mathrm{n}=311$ Kurse

Insgesamt wurden rd. zwei Drittel der Kursbesuche (65\%) erfolgreich abgeschlossen (siehe Grafik 46). Bei den befragten Jugendlichen mit Migrationshintergrund war der Anteil der erfolgreichen Kursabschlüsse höher als bei den KundInnen aus Österreich (68\% vs. $55 \%$ ). Eine hundertprozentige Erfolgs- bzw. Abschlussquote erzielten die Deutschkurse, gefolgt von den Ausbildungen zum Erwerb des Hauptschulabschlusses und den Büro- und EDV-Kursen mit rd. $80 \%$ erfolgreich abgeschlossenen Maßnahmenteilnahmen. Hingegen führten nur ein Drittel der besuchten Lehr- und Berufsausbildungen bis zum Erhebungszeitpunkt zu einem erfolgreichen Berufsabschluss, da sich diese über einen längeren Zeitraum erstrecken und höhere Ausdauer erfordern. 


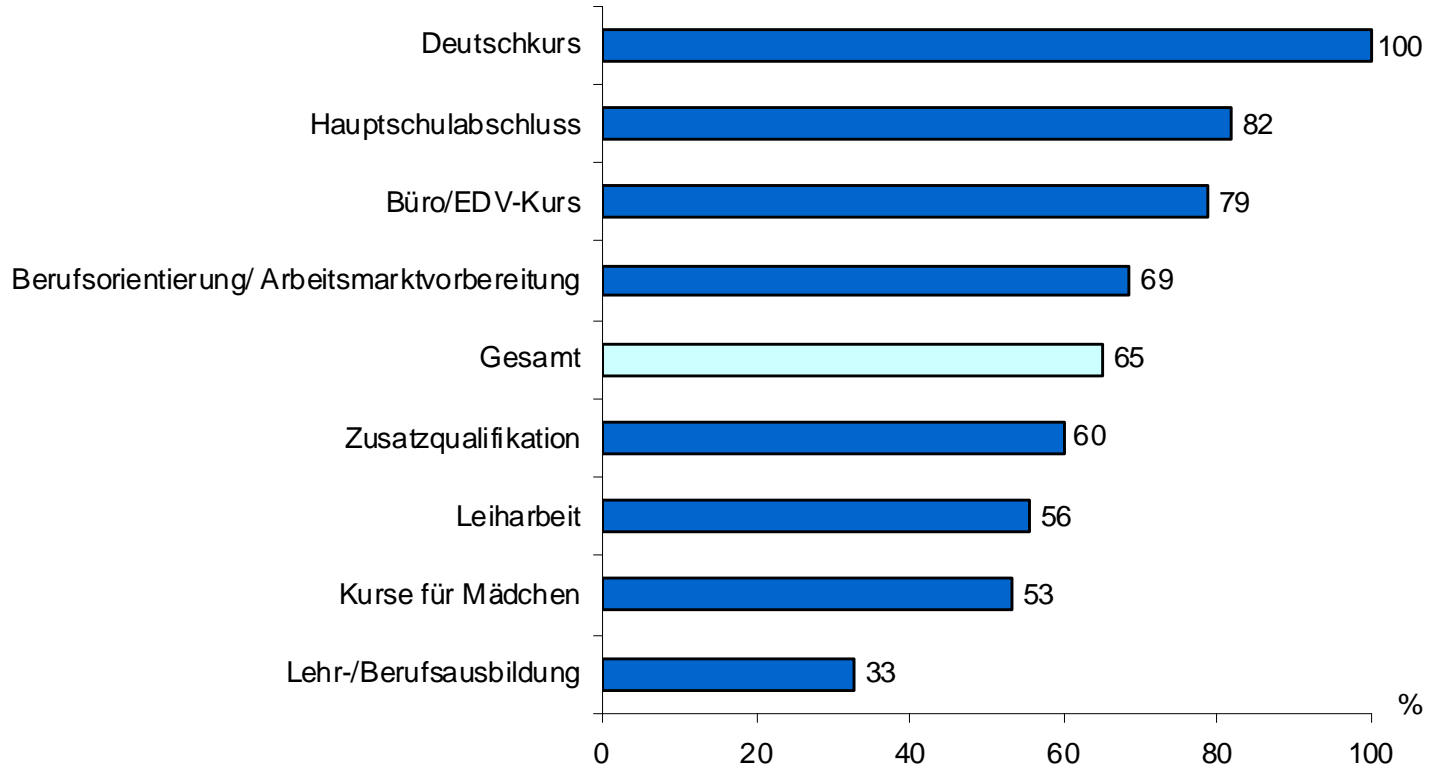

${ }^{1}$ Grundlage bilden jeweils die Anzahl der Kursbesuche (nach Kursart), da jede/r Kundln zu den letzten zwei Kursen detaillierte befragt wurde.

Quelle: KMU FORSCHUNG AUSTRIA, Befragung der jugendlichen AMS-KundInnen, $n=313$ Kurse

Etwa ein Drittel der Kursbesuche wurde nicht erfolgreich beendet. Als Gründe werden vorwiegend Probleme mit den TrainerInnen oder in der Berufsschule sowie das Nichteinhalten der vorgegebenen Rahmenbedingungen (Fehlzeiten, Unpünktlichkeit) genannt, die zu Kündigungen oder Beendigungen des Ausbildungsverhältnisses führten. Dies trifft vor allem auf Lehr- und Berufsbildungen im Auftrag des AMS zu. Oftmals hat der Kurs auch nicht den Interessen und Vorstellungen der Jugendlichen entsprochen, weshalb sie von einem weiteren Besuch absahen. Dabei spielt auch der erste Eindruck von der Kursmaßnahme eine entscheidende Rolle, wie ein Jugendlicher berichtet: „Danach war ich in einem JASG-Lehrgang für Kellner, das war ein Blödsinn. Ich bin hingegangen 20 Minuten, danach habe ich gesagt, nein, ich will das nicht und ich bin rausgegangen. Die waren so arrogant und wollten einen kränken." Einige KundInnen haben die Qualifizierungsaktivitäten abgebrochen, weil sie eine Lehrstelle oder einen Arbeitsplatz am freien Arbeitsmarkt finden konnten. Aber auch Krankenstände und Auslandsaufenthalte hatten die Beendigung der Ausbildungsaktivitäten zur Folge. Außerdem können einige wenige KundInnen noch keinen erfolgreichen Abschluss vorweisen, da sie sich noch in laufenden Maßnahmen befinden.

Insgesamt werden $63 \%$ der Kursbesuche als nützlich erlebt. Jugendliche mit Migrationshintergrund (66\%) empfinden ihre Maßnahmenteilnahmen generell nützlicher als KundInnen aus Österreich (53 \%). Damit wird die Nützlichkeit der Kurse von Seiten der KundInnen mit Migrationshintergrund (69\%) ähnlich hoch bewertet wie bei der Erhebung im Jahr 2007 (vgl. Heckl et al., 2007). Vor allem jene Kurse, die erfolgreich abgeschlossen wurden, werden als sehr oder eher nützlich befunden (75\%), während dies "nur" auf $40 \%$ der Maßnahmenteilnahmen zutrifft, die zu keiner erfolgreichen Beendigung führten. Von den Befragten mit Migrationshintergrund werden sowohl die erfolgreich abgeschlossenen (77\%) als auch die nicht zu Ende geführten Kurse (42\%) als nützlicher eingestuft als von den ÖsterreicherInnen (68 \% bzw. $34 \%)$. 
Aber auch die Art des Zustandekommens der Kursbesuche hat einen Einfluss auf die Beurteilung der Nützlichkeit. Insgesamt wurden die Kursteilnahmen, die auf eigenen Wünschen beruhten (70 \%) noch nützlicher eingestuft als Kursbesuche, die gemeinsam erarbeitet (62\%) wurden oder die der/die Beraterln (60\%) initiierte. MigrantInnen bewerten wiederum die Kurse aus eigenen Wünschen (73\%) nützlicher als österreichische Jugendliche (63\%), ebenso wie Maßnahmen, die gemeinsam erarbeitet $(65 \%$ vs. $50 \%$ ) sowie jene, die von dem/r Beraterln vorgeschlagen wurden (65\% vs. $48 \%$ ).

Ebenso werden die verschiedenen Themen der Qualifizierungsmaßnahmen unterschiedlich nützlich bewertet, wie Grafik 47 zeigt. Insbesondere Deutsch- und Hauptschulabschlusskurse werden - u. a. bedingt durch die hohe Erfolgsquote - als sehr nützlich erlebt. Aber auch Lehr- und Berufsausbildungen sowie mädchenspezifische Maßnahmen werden bei jeweils knapp zwei Drittel der Kursbesuche als nützlich eingestuft. Bei den Fokusgruppen wird ebenfalls die hohe Zufriedenheit mit Lehrausbildungen in Ausbildungseinrichtungen deutlich. Die Jugendlichen haben teilweise den Eindruck mehr zu lernen als bei einer Lehrstelle in einem Betrieb, wie ein junger Mann erzählt: „Man lernt hier schon Sachen im 1. Lehrjahr, die andere erst im 3. Lehrjahr lernen, was ein Geselle macht.“ Auch ein Kfz-Lehrling pflichtet dem bei: „Bei uns wird im 1. und 2. Lehrjahr nur geputzt und da [in der Einrichtung] haben wir nach der 2. Woche schon Getriebe zerlegen müssen." Ein anderer Lehrling, der seine Ausbildung länger in einem Unternehmen absolvierte, bevor er diese bei einer Einrichtung fortsetzte, hat jedoch auch die Erfahrung gemacht, dass die Unternehmen andere praktische Anforderungen an die Lehrlinge stellen, die bei den Ausbildungseinrichtungen möglicherweise zu kurz kommen: „Es ist aber auch umgekehrt, ein Lehrling im 3. Lehrjahr ist vom Betrieb nach Hause geschickt worden, weil er nicht stemmen konnte, weil er es hier [in der Einrichtung] nie gemacht hat."

Eine geringere Nützlichkeit wird vor allem im Bereich der technischen und sprachlichen Zusatzqualifikationen wahrgenommen. Rd. $60 \%$ dieser Kursbesuche werden als weniger oder überhaupt nicht nützlich eingestuft, wobei Jugendliche mit Migrationshintergrund diese noch weniger nützlich empfinden als österreichische Kundlnnen. Auch Büro- und EDV-Kurse, denen in $41 \%$ der Fälle eine geringere Nützlichkeit bescheinigt wird, werden von MigrantInnen als weniger nützlich erlebt als von Jugendlichen ohne Migrationshintergrund. Berufsorientierungsmaßnahmen und Aktivitäten zur Vorbereitung auf den Arbeitsmarkt werden in $43 \%$ der Fälle als weniger oder nicht nützlich beurteilt. Vor allem österreichische Jugendliche bewerten diese Kursbesuche deutlich weniger nützlich als MigrantInnen (56 \% vs. $38 \%$ ). Dies könnte darauf zurückzuführen sein, dass die migrantische Zielgruppe mehr Unterstützungsbedarf beim Berufswahlprozess und Einstieg in den Arbeitsmarkt hat.

Bei den Berufsorientierungsmaßnahmen ist vor allem der BOCO-Kurs bei den Jugendlichen umstritten, wie auch die Erzählungen aus den Fokusgruppen zeigen. Manche Jugendliche sind davon begeistert: „BOCO war leiwand. Die Trainer sind extrem leiwand." Anderen hat dieser Kurs weniger gefallen, wie ein Jugendlicher berichtet: „Der BOCO-Kurs ist unnütz. Beim BOCO-Kurs wird von jedem Beruf erklärt, was man macht. Es war jeden Tag 4-5 Stunden, jeden Tag das Gleiche." 
Manchmal sind die KundInnen auch mit den Anforderungen im Kurs überfordert und haben Schwierigkeiten, den Ausführungen der TrainerInnen zu folgen, wie ein Mädchen erzählt: „Der BOCO-Kurs war am schlechtesten. Mir hat gar nichts dort gefallen. Das ist schlimmer als Schule sogar. Man musste Bewerbungen schreiben, Lebenslauf, das war eh ganz gut. Dann hat sie [die Trainerin] die ganze Zeit geredet, ich bin nicht mitgekommen, was sie gesagt hat. Wir haben auch Ausflüge gemacht, aber das war gar nichts für mich, es war zu langweilig."

Grafik 47 Bewertung der letzten zwei Kurse nach deren Nützlichkeit, Anteil der Kursbesuche in Prozent ${ }^{1}$

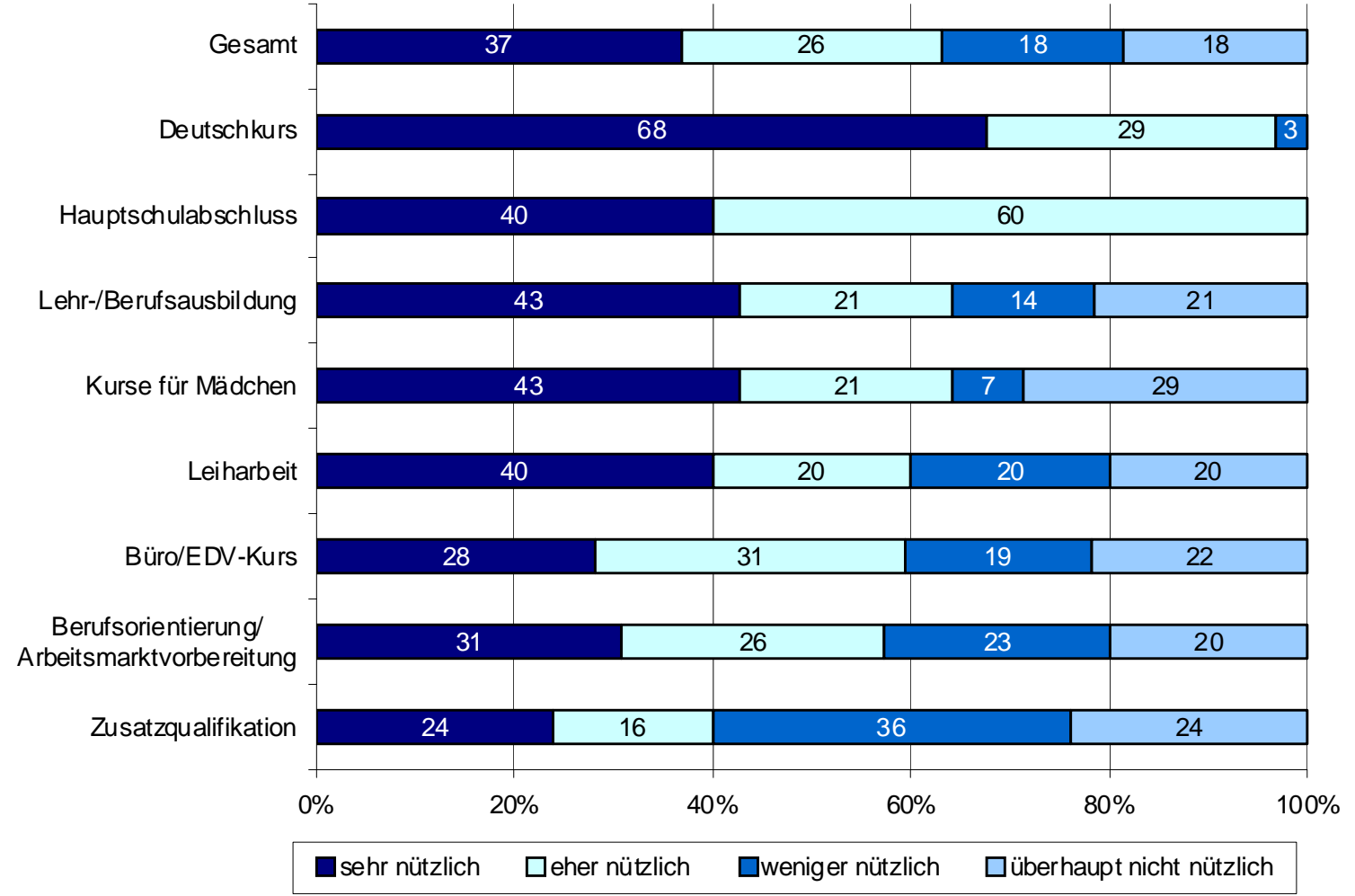

${ }^{1}$ Grundlage bilden jeweils die Anzahl der Kursbesuche (nach Kursart), da jede/r Kundln zu den letzten zwei Kursen detaillierte befragt wurde.

Quelle: KMU FORSCHUNG AUSTRIA, Befragung der jugendlichen AMS-KundInnen, $n=314$ Kurse

Bei der Bewertung der Nützlichkeit der Kursbesuche sind auch Unterschiede zwischen jugendlichen und erwachsenen AMS-KundInnen zu beobachten. Während die befragten erwachsenen MigrantInnen in den Regionalen Geschäftsstellen Huttengasse und Laxenburger Straße sowohl die Besuche von Fremdsprachenkursen als auch von technischen Zusatzausbildungen sowie von Büro- und EDV-Schulungen als sehr nützlich empfanden, stufen die Jugendlichen mit Migrationshintergrund ihre Teilnahmen an diesen Qualifizierungsmaßnahmen tendenziell weniger nützlich ein. Hingegen finden die Coaching- und Orientierungsmaßnahmen bei der jugendlichen Klientel trotz gewisser Kritikpunkte mehr Anklang als bei den Erwachsenen. Die befragten erwachsenen MigrantInnen in den Regionalen Geschäftsstellen Huttengasse und Laxenburger Straße haben jeweils etwa die Hälfte ihrer Besuche von Coaching- und Orientierungsmaßnahmen als weniger oder nicht nützlich eingestuft, jedoch trifft dies nur auf $38 \%$ der Jugendlichen mit Migrationshintergrund zu (vgl. Heckl et al., 2009). Damit wird der unterschiedliche Bedarf an Qualifizierungsmaßnahmen zwischen jugendlichen und 
erwachsenen AMS-KundInnen deutlich. Vor allem für jugendliche MigrantInnen erscheinen berufs- und arbeitsmarktvorbereitende Aktivitäten zielführend, während im Erwachsenenalter stärker der Bedarf nach konkreten (Zusatz-) Ausbildungen besteht.

\subsubsection{Allgemeine Zufriedenheit und Wünsche an das AMS}

Insgesamt ist die Zufriedenheit der befragten KundInnen mit dem Wiener AMS Jugendliche sehr hoch. $85 \%$ der jugendlichen Zielgruppe sind mit den Serviceleistungen des AMS insgesamt sehr oder eher zufrieden. Kundlnnen aus Österreich sind sogar noch zufriedener mit dem AMS als Jugendliche mit Migrationshintergrund (91\% vs. $83 \%$ ), wie aus Grafik 48 ersichtlich. Ein junger Mann mit Migrationshintergrund betont: „Das AMS ist schon eine Hilfe, weil diese Möglichkeiten findet man nicht überall. Das AMS kümmert sich um uns, das finde ich ok. Die wollen das wir arbeiten, damit wir für später etwas haben." Auch bei Jugendlichen, die noch keine Arbeitserfahrung vorweisen können und Unterstützung beim Eintritt in den Arbeitsmarkt benötigen, ist die Zufriedenheit mit den Leistungen des AMS noch höher als bei KundInnen, die bereits berufliche Praxis gesammelt haben ( $89 \%$ vs. $84 \%$ ). Bei den Mädchen ist der Anteil der „sehr Zufriedenen" höher als bei den Burschen (44\% vs. $35 \%$ ), ebenso zeichnen sich die Jugendlichen unter 16 Jahren durch eine sehr hohe Zufriedenheit (48\%) aus. Mit zunehmender Vormerkdauer und damit negativen Erfahrungen bei der Arbeits- bzw. Lehrstellensuche geht die Zufriedenheit leicht zurück: Der Anteil der „sehr Zufriedenen“ sinkt von $46 \%$ bei KundInnen, die erst seit 0-2 Monaten vorgemerkt sind, auf $36 \%$ bei jenen, die länger als $1 \mathrm{Jahr}$ arbeitslos sind. Auch insgesamt reduziert sich die Zufriedenheit von $89 \%$ bei KundInnen mit einer sehr kurzen Arbeitslosigkeit auf $80 \%$ bei einer mehr als einjährigen Vormerkdauer, bleibt aber damit immer noch sehr hoch.

Die Jugendlichen sind mit dem AMS so zufrieden, weil sie Hilfe und Unterstützung bei der Suche nach einer Arbeits- oder Lehrstelle sowie wertvolle Informationen für den Bewerbungsprozess erhalten und Qualifizierungsmaßnahmen besuchen können. Auch der neue Standort, die schönen Räumlichkeiten, die gute Erreichbarkeit, die Verbesserungen beim Terminvereinbarungssystem, die kürzeren Wartezeiten sowie das Angebot an PCs tragen zur Zufriedenheit der befragten KundInnen bei.

Lediglich $15 \%$ der befragten Jugendlichen sind mit dem AMS weniger oder nicht zufrieden. Als Kritikpunkte führen sie an, dass sie nicht ausreichend Unterstützung bei der Suche nach einer Arbeit oder einer Lehrstelle bzw. keine passenden, aktuellen freien Stellenanzeigen erhalten. Sie beklagen sich, dass sie in Kurse geschickt werden, die ihnen uninteressant oder für sie nicht adäquat erscheinen, während eigene Kurswünsche nicht realisiert werden können. Einzelne Jugendliche beschweren sich über die Häufigkeit der Termine (werden einerseits als zu selten und andererseits als zu häufig wahrgenommen), über die neue Terminvereinbarungsregelung, die bei Verspätungen ein neuerliches Aufsuchen des AMS erfordern kann, und dass sie zu wenig Geld vom AMS bekommen.

Insbesondere der erste AMS-Besuch ist für einige Jugendliche schwierig, da sie sich mit den Anforderungen, die dort an sie gestellt werden (wie z. B. Formulare ausfüllen) überfordert fühlen. Ein Jugendlicher erzählt bei einer Fokusgruppe: „Beim AMS sind sie ur-schlecht zu mir gewesen. Ich habe irgendetwas falsch ausgefüllt und die Frau schreit mich gleich an." Auch die Fülle an Informationen überschreitet häufig die Aufnahmefähigkeit der KundInnen, wie ein andere Bursch berichtet, dem das Lehrstellenregister mit Ausbildungsmöglichkeiten mitgegeben wurde: „Das war extrem, das war so dick wie ein Buch. Da sind so Firmen gestanden und Nummern, aber alles so 
klein gedruckt, das habe ich lesen müssen." Vor allem wenn die KundInnen alleine das AMS aufsuchen müssen, haben sie Schwierigkeiten sich zurecht zu finden, wie folgende Aussage verdeutlicht: „Ich bin dort alleine hingegangen und wie ich alleine dort war, habe ich nicht einmal Hilfe bekommen. Dann bin ich noch ein zweites Mal mit meiner Mutter hingekommen und dann habe ich aber den Zettel bekommen." Auch wenn die Jugendlichen erst seit kurzem in Österreich sind, haben sie auf Grund mangelnder Sprachkenntnisse Unbehagen, das AMS aufzusuchen, wie ein Mädchen, das erst vor kurzem aus Tschetschenien nach Österreich gekommen ist, erzählt: „Das erste Mal beim AMS war komisch, du kennst die Leute nicht, du weißt nicht, du kannst nicht sprechen [auf Deutsch]. Nach dem [Deutsch-]Kurs war es ok. Am Anfang ist es schwierig, wenn man nicht viel versteht."

Grafik 48 Zufriedenheit mit dem AMS insgesamt, Anteil der befragten Jugendlichen nach Migrationshintergrund in Prozent

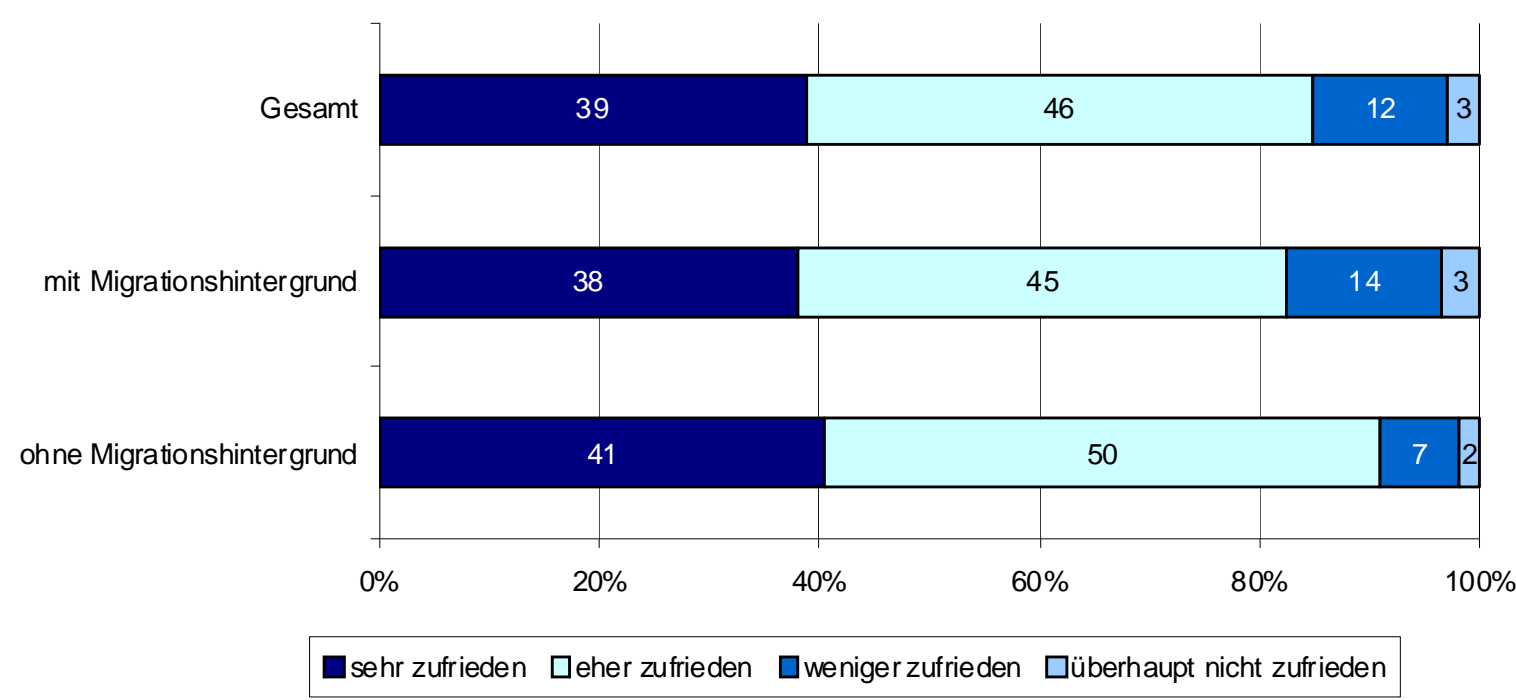

Quelle: KMU FORSCHUNG AUSTRIA, Befragung der jugendlichen AMS-KundInnen, $n=403$

Für die Zukunft wünschen sich die Jugendlichen vom AMS in erster Linie eine Arbeit oder eine Lehrstelle vermittelt zu bekommen, wie insgesamt $63 \%$ der Befragten angeben. Der gezielte Wunsch nach einem freien Arbeitsplatz wird von $35 \%$ erwähnt (siehe Grafik 49). Bei KundInnen aus Österreich ist der Bedarf nach einer Arbeit noch stärker ausgeprägt als bei MigrantInnen (39\% vs. $35 \%$ ). Vor allem ältere Jugendliche (42\% der 19-Jährigen bzw. $48 \%$ der über 20-Jährigen), Kundlnnen mit Arbeitserfahrung (41\%) und Burschen (38\%) sind an einer Vermittlung einer Arbeit interessiert. Diese Jugendlichen äußern den Wunsch, möglichst rasch eine für sie passende Arbeitsstelle zu finden. Darüber hinaus hofft mehr als ein Viertel der Befragten (27\%) auf eine Lehrstelle, wobei sich mehr Jugendliche mit Migrationshintergrund nach einem freien Ausbildungsplatz sehnen als österreichische KundInnen (29\% vs. $22 \%)$. Der Wunsch nach einer Lehrstelle ist insbesondere bei jüngeren Jugendlichen (60\% der unter 16Jährigen und $47 \%$ der 17-Jährigen), den Befragten ohne Arbeitserfahrung (43\%) sowie bei männlichen Jugendlichen (31 \%) stärker ausgeprägt. 
$16 \%$ der KundInnen wünschen sich eine höhere finanzielle Unterstützung vom AMS. Dies trifft vor allem auf MigrantInnen (18\%), Burschen (22\%), ältere Jugendliche ab 18 Jahren und KundInnen mit Arbeitserfahrung (19\%) zu. Auch mit der Dauer der Arbeitslosigkeit steigt der Bedarf nach einer höheren finanziellen Unterstützung. Während nur $13 \%$ der KundInnen, die erst seit maximal 2 Monaten beim AMS vorgemerkt sind, den Wunsch nach mehr Geld äußern, ist beinahe einem Viertel (23\%), die bereits länger als ein Jahr beim AMS vorgemerkt sind, eine höhere finanzielle Unterstützung ein Anliegen.

Optimierungsbedarf wird von $14 \%$ der Jugendlichen im Bereich der Beratungs- und Unterstützungsangebote des AMS gesehen. Dieser Wunsch wird von Mädchen (20\%) und KundInnen mit Migrationshintergrund (15\%) deutlich häufiger erwähnt als von Burschen (8\%) und Jugendlichen ohne Migrationshintergrund (9\%). Diese KundInnen wollen mehr Unterstützung und Hilfestellung bei der (Lehr-)Stellensuche sowie mehr freie Stellenangebote von den BeraterInnen übermittelt bekommen. Außerdem wünschen sie sich, dass die AMS-BeraterInnen freundlicher und hilfsbereiter sind, weniger Druck ausüben und sich intensiver mit den Anliegen und Wünschen der KundInnen auseinander setzen, wie auch folgendes Zitat verdeutlicht: „Dass die Berater zuhören, was man will und nicht nur bestimmen, was man tun muss." Darüber hinaus sind bessere, gezieltere Beratungsgespräche gefragt, die ausführlicher über berufliche Möglichkeiten und Qualifizierungsoptionen informieren, genügend Zeit und eine richtige Gesprächsatmosphäre bieten, wie folgendermaßen erläutert wird „Die Berater sollten besser zuhören und einen anschauen, wenn sie sprechen und nicht nur am Computer tippen." Auch ein Jugendlicher wünscht sich bei einer Fokusgruppe, „dass sie freundlicher auf einen zugehen und dass sie gleich ein bisschen helfen und Tipps geben, wo man hingehen kann und was man machen kann. "Auch eine rasche Hilfeleistung ist gefragt, wie ein Bursch erwähnt: „Heute bin ich da und ich will wissen, was ich jetzt oder nächste Woche anfangen kann, aber das machen sie leider nicht." Vereinzelt wird auch Interesse an einer faireren, nicht auf die Herkunft bezogenen Behandlung sowie an Beratungsgesprächen in anderen Sprachen bekundet.

$13 \%$ der Befragten sind bestrebt, einen Kurs oder eine Ausbildung zu absolvieren. Dabei sollen in erster Linie spezifische Qualifizierungswünsche erfüllt werden, vereinzelt wird auch der Bedarf nach mehr und besseren Ausbildungsplätzen geäußert. Insbesondere KundInnen mit Migrationshintergrund (14\%), Mädchen (19\%), ältere KundInnen (14\% der 19-Jährigen bzw. $17 \%$ der Über-20-Jährigen), Jugendliche ohne Arbeitserfahrung (20\%) und KundInnen, die bereits länger als ein Jahr arbeitslos sind (20\%), haben Interesse an einer Ausbildung mit Unterstützung durch das AMS.

$13 \%$ der KundInnen sind zufrieden und haben keine weiteren Wünsche an das AMS. Dies trifft vor allem Jugendliche mit Migrationshintergrund (16\%) und KundInnen, die erst maximal seit 2 Monaten beim AMS vorgemerkt sind (16\%), zu.

Sonstige Wünsche, die von $5 \%$ der befragten Jugendlichen erwähnt werden, betreffen u. a. die Verbesserung der AMS-Homepage, den Terminvereinbarungsprozess und kürzere Wartezeiten. Einzelne KundInnen sind nur an ihrer Weiterversicherung interessiert und wollen vom AMS möglichst „in Ruhe gelassen werden“ und keinen (weiteren) Kurs mehr besuchen. 
Grafik 49 Wünsche an das AMS, Anteil der befragten Jugendlichen nach Migrationshintergrund in Prozent

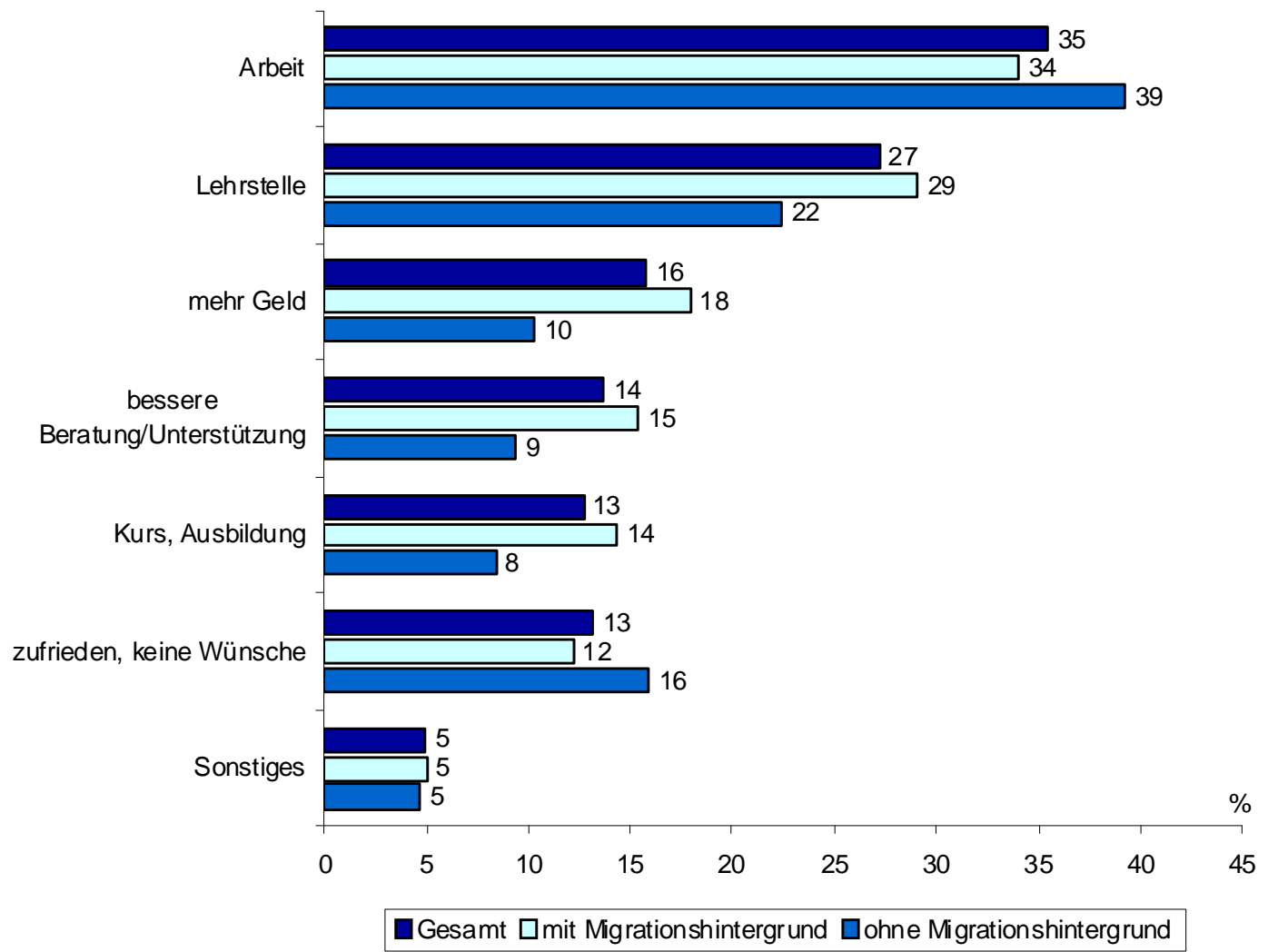

Quelle: KMU FORSCHUNG AUSTRIA, Befragung der jugendlichen AMS-KundInnen, $\mathrm{n}=386$, Mehrfachnennungen möglich

Im Vergleich zur Erhebung im Jahr 2007 hat bei den KundInnen mit Migrationshintergrund vor allem der Wunsch nach einer Arbeit und einer Lehrstelle deutlich an Bedeutung gewonnen. Während sich 2007 „nur“ rd. ein Drittel der befragten MigrantInnen vom AMS eine Arbeit oder eine Lehrstelle wünschten, trifft dies nun auf $63 \%$ der Jugendlichen mit Migrationshintergrund zu. Möglicherweise gewinnt für die Jugendlichen $-u$. a. bedingt durch die wirtschaftlich schwierigere Situation - die rasche Vermittlung auf einen Arbeits- oder Ausbildungsplatz an Priorität. Hingegen hat der Wunsch nach einer besseren Beratung an Relevanz verloren. Im Jahr 2007 war es noch für rd. $23 \%$ der Jugendlichen ein Anliegen, eine bessere Betreuung durch die BeraterInnen zu erhalten, während dieses 2010 nur mehr von $15 \%$ der KundInnen mit Migrationshintergrund vorgebracht wird. Auch der Wunsch nach kürzeren, angenehmeren Wartezeiten und -räumlichkeiten, der 2007 noch von einem Zehntel der MigrantInnen erwähnt wurde, ist abgesehen von vereinzelten Meldungen kaum mehr präsent. (vgl. Heckl et al., 2007) Damit wird deutlich, dass sich durch den Umzug in das neue AMS-Gebäude am Gumpendorfer Gürtel und die organisatorischen Veränderungen sowohl die Rahmenbedingungen als auch die Qualität der Beratungsgespräche für die KundInnen verbessert haben. 


\section{Schlussfolgerungen}

Im Wiener AMS Jugendliche gab es weitreichende organisatorische Neuerungen, die von der Mehrheit der KundInnen und auch der BeraterInnen begrüßt werden. Auch wurde die Implementierung des Diversity Management Grundsatzes auf allen Ebenen der Organisation vorangetrieben und viele Maßnahmen gesetzt, um der Zusammensetzung der Klientel, die eben zum überwiegenden Teil aus MigrantInnen besteht, gerecht zu werden. Daraus ergibt sich eine relativ hohe Zufriedenheit mit dem AMS insgesamt, obgleich das AMS oftmals nur bedingt als hilfreich erlebt wird, da eben keine Vermittlung auf eine Lehrstelle oder in einen Job aus dem AMS-Besuch resultiert. Das AMS kann sein Ziel, eine erfolgreiche Integration seiner Klientel auf dem Arbeitsmarkt, auf Grund verschiedenster Rahmenbedingungen nur teilweise erreichen. Es agiert im Spannungsfeld eines Arbeitsmarktes, auf dem immer höhere Qualifikationen gefragt sind und einer Klientel, die diesen Ansprüchen immer weniger gerecht werden. Vor allem bei MigrantInnen kumulieren die Problemlagen, indem zu niedriger Qualifikation noch Sprachschwierigkeiten und kulturelle Barrieren kommen und eine Integration am Arbeitsmarkt erschweren.

Obwohl das AMS die Rahmenbedingungen nicht unmittelbar ändern kann, so können dennoch im Rahmen des Betreuungs- und Beratungsprozesses und in Hinblick auf nachgelagerte Dienstleistungen sowie in Kooperation mit anderen zentralen Akteuren Entwicklungen gefördert werden, die der besseren Integration von Jugendlichen im Allgemeinen und MigrantInnen im Besonderen am Arbeitsmarkt förderlich sind und die Leistungen des AMS verbessern helfen. Im Folgenden seien verschiedene Bereiche erwähnt, die Möglichkeiten zur Optimierung bieten.

\section{Beratung}

Eine qualitativ hochwertige Beratung kostet vordergründig Zeit und Flexibilität. Im Moment sind 15 Minuten für ein Beratungsgespräch vorgesehen. Jedoch bindet der Verwaltungsaufwand stark die Ressourcen der AMS-MitarbeiterInnen, denn die Daten der KundInnen müssen erhoben und die Betreuungsabläufe dokumentiert werden. Damit wird viel Zeit aufgewandt, um quantifizierbare Größen zu erfassen, und wertvolle Beratungszeit geht verloren. Auch stören eingehende Telefongespräche die Beratung selbst. Neben den Telefonanrufen binden auch die zahlreichen zu beantwortenden EMails, die u. a. durch die Forcierung des e-AMS-Kontos immer mehr werden, die Kapazitäten der MitarbeiterInnen. Um den Trend in Richtung kürzere und schlechtere Beratungen einzudämmen, gilt es daher die Qualität der Beratung stärker zu forcieren und die quantitative Erfassung wieder etwas in den Hintergrund zu drängen, sowie den MitarbeiterInnen die Möglichkeit zu bieten, sich dem/der Kunden/in ungestört zu widmen. Zur Steigerung der Beratungsqualität sollen die Qualifikationen und Ressourcen, die die Mitarbeiterlnnen mitbringen (wie z. B. pädagogische Ausbildungen) stärker genutzt werden. Dieses Know-how kommt im Rahmen der Kundengespräche zu wenig zum Einsatz, wenn die Quantifizierung zu sehr im Vordergrund steht. Eine Erweiterung der Beratungszeit wäre durch eine Erhöhung der personellen Ressourcen möglich. Es könnten z. B. häufiger zwei reguläre Kundentermine zu einem längeren Gesprächstermin von einer halben Stunde zusammengefasst werden.

Jedoch ist nicht nur die Beratungsqualität, sondern auch die Gewährleistung einer langfristigen, kontinuierlichen Betreuung für den Vermittlungserfolg wesentlich. Viele KundInnen des AMS Jugendliche ohne Leistungsbezug halten ihre Termine nicht ein und melden sich beim AMS immer wieder an und ab. Dadurch geht wertvolle Be- 
treuungszeit verloren, in der kaum ein Fortschritt für die Vermittlung am Arbeitsmarkt erreicht wird. Die BeraterInnen würden sich daher mehr Kooperation von Seiten der Jugendlichen bei der Termineinhaltung bzw. strengere Vorgaben von Seiten des AMS wünschen, um mehr Betreuungskontinuität zu gewährleisten.

Eine kontinuierliche Betreuung und der Aufbau eines Vertrauensverhältnisses zwischen Kunde/in und Beraterln wird auch erleichtert, wenn die AMS-BeraterInnen in engerem Kontakt zu Kursanbietern stehen und im Beratungsgespräch mit diesen Unklarheiten besprechen und den KundInnen nähere Informationen zu den Kursen bieten können. Daher sollten die Kurs- und Beratungseinrichtungen für die AMS-MitarbeiterInnen telefonisch erreichbar sein, damit sie sich bei Informations- und Klärungsbedarf im Rahmen eines Kundengespräches aktiv an die Einrichtungen wenden können.

\section{Kurse und Qualifizierung}

Neben der Jobvermittlung steht insbesondere im AMS Jugendliche die Weiterqualifizierung der KundInnen im Fokus, um die großen Ausbildungsdefizite zu minimieren, die Employability zu erhöhen und die nachhaltige Vermittelbarkeit in den Arbeitsmarkt zu gewährleisten. Der Organisation der Kurs- und Qualifizierungsmaßnahmen kommt daher große Bedeutung zu. Dies betrifft einerseits die organisatorischen Rahmenbedingungen der Kursgestaltung sowie auch inhaltliche Verbesserungen oder gezielte Änderungen einzelner Maßnahmen.

Ein umfangreiches Kursangebot und nicht „more of the same“ würde es ermöglichen, den KundInnen möglichst vielfältige und passgenauere Qualifizierungsoptionen anbieten zu können. Auch häufigere Kurseinstiege sind insbesondere bei längeren Ausbildungen und Jahreskursen gefragt. Dort sollte nicht nur ein Einstiegstermin geboten werden, sondern halbjährlich oder quartalsweise weitere Kurse, um einen flexibleren Eintritt zu gewährleisten und den KundInnen nach kurzfristigen Ausfällen (z. B. auf Grund von Krankheit oder einem kurzen Dienstverhältnis) einen Alternativtermin in zeitlicher Nähe anbieten zu können. Ein weiteres Problem bezüglich der zeitlichen Gestaltung bei Kursmaßnahmen ist, dass oftmals viel Zeit verstreicht bis ein Kurs freigegeben und KundInnen zugebucht werden können. Dies ist vor allem dann suboptimal, wenn der Kursbeginn bereits ein oder zwei Wochen nach dem Aufruf zu Zubuchungen angesetzt ist, und somit nicht mehr alle Jugendlichen erreicht werden können, für die die geplante Maßnahme passend wäre, da die KundInnen durchschnittlich nur einmal pro Monat das AMS aufsuchen. Gleichzeitig sollte der Zeitraum zwischen Infotag und Kursbeginn möglichst kurz sein, um eine „nahtlose“ Fortsetzung der Maßnahmen zu ermöglichen.

Um die Kurse zu füllen, buchen die AMS-MitarbeiterInnen meist mehr KundInnen zu einem Kurs zu als Plätze angeboten werden. Dadurch kann es vorkommen, dass einige Jugendliche keinen freien Platz mehr in der ausgewählten Kursmaßnahme erhalten. In solchen Fällen werden die KundInnen meist auf eine Warteliste gesetzt. Jedoch ist dann weder für den/die AMS-Beraterln noch für den/die Kunden/in abschätzbar, wie lange es dauern wird, bis er/sie in den Kurs einsteigen kann, da ein Platz z. B. erst wieder nach einer erfolgreichen Vermittlung frei wird.

Auch die TeilnehmerInnenselektion durch die Kurseinrichtungen im Vorfeld einer Maßnahme wird von Seiten des AMS als wenig förderlich erlebt. Die Trägereinrichtungen, die am Vermittlungserfolg gemessen werden, wählen in erster Linie jene KundInnen aus, die die besten Chancen am Arbeitsmarkt haben (Creaming). Aber vor 
allem für KundInnen mit den schlechtesten Vermittlungschancen ist die Unterstützung unabdingbar. D.h. auch für „schwache“ Jugendliche mit vielfältigen Defiziten soll ein entsprechendes Maßnahmenangebot zur Verfügung stehen und die Erfolgsmessung diese erschwerten Bedingungen berücksichtigen. In diesem Zusammenhang ist der starke Fokus auf den direkten Vermittlungserfolg zu hinterfragen, da sich der Erfolg bei schwierigen Zielgruppen möglicherweise nicht unmittelbar, sondern erst auf lange Sicht einstellt.

Weiterer Optimierungsbedarf wird bezüglich der Kursinformationen gesehen. Dies betrifft sowohl die Kursorganisation als auch -inhalte. KundInnen benötigen häufig einen Anfahrtsplan, wie sie das Kursinstitut erreichen können und Ansprechpersonen bzw. Telefonnummern, damit sie nachfragen können, wenn sie Orientierungsprobleme haben. Aber auch die Motivation zur Kursteilnahme kann gesteigert werden, wenn der/die Jugendliche umfassendere inhaltliche Vorstellungen über den Kurs erhält. Das Informationsmaterial sollte sich daher nicht ausschließlich auf den Infotag beziehen, sondern auch Informationen über den geplanten Kurs beinhalten, damit sich die Jugendlichen schon im Vorfeld darauf einstellen können, was sie im Rahmen der Maßnahme erwarten wird.

Auch bezüglich der Zielgruppenausrichtung der Kursmaßnahmen ist Vorsicht geboten: Eine Kursmaßnahme sollte sich nicht an eine zu breite Zielgruppe richten, da dies die Gruppenbildung erschwert und die Möglichkeiten der aktiven Mitwirkung reduziert. Eine Zuteilung homogener Gruppen in einen bestimmten Kurs erleichtert die Ausrichtung der Kursinhalte und ermöglicht ein Aufbauen auf ähnlichem Qualifikationsniveau. Gleichzeitig sollten die Kurse nicht zu spezifisch ausgerichtet sein und sich nur an einen speziellen Personenkreis, der nicht über unterschiedliche Qualifikationen oder Lerntypen definiert ist (wie z. B. Mädchen mit Migrationshintergrund), wenden, um andere Zielgruppen, die sich z. B. auch für diese Qualifizierung (z. B. zur Kindergartenhelferln) interessieren, nicht zu diskriminieren und davon auszuschließen. Dies betrifft häufig Burschen, da sich einige Ausbildungen speziell an der weiblichen Zielgruppe orientieren. Ebenso ist die Gruppengröße bei einigen Maßnahmen zu groß. Die Betreuung von 25 „schwierigen“ Jugendliche, die relativ individuelle Hilfestellungen benötigen, überfordert die TrainerInnen häufig. Bei großen Gruppen steigt auch das Aggressions- und Konfliktpotenzial, so dass die Kurszeit oftmals von Disziplinierungsmaßnahmen überschattet ist.

Ein weiteres Thema, das bereits am AMS Jugendliche aktuell ist, ist die Kinderbetreuung während des Kursbesuchs. Insbesondere Kurse, die sich an Mädchen oder Wiedereinsteigerinnen richten, sollten Kinderbetreuungsangebote beinhalten, um der Zielgruppe den Kursbesuch leichter zu ermöglichen und in der Folge den Eintritt in die Arbeitswelt zu forcieren.

Außerdem sollte der generelle „Kurszwang“ hinterfragt werden. Die Kurse werden auch eingesetzt, um Übertritte in die Langzeitarbeitslosigkeit zu vermeiden und entsprechen oft keinem individuellen Weiterbildungsplan, sondern richten sich vielmehr nach dem Kursplatzangebot. Dieser Kurszwang wird von Kundlnnenseite oft negativ empfunden, wenn beispielsweise bereits drei Monate nach einem Kursbesuch eine weitere Maßnahme besucht werden muss, die oftmals weder in Zusammenhang mit den Berufswünschen der KundInnen noch einem kontinuierlichen Qualifizierungspfad stehen. Es ist daher fraglich, ob diese „verpflichtenden“ Kurse die Motivation der KundInnen und damit die Vermittlung und Integration in den Arbeitsmarkt fördern. 
Auch in Bezug auf spezielle Kursmaßnahmen und Kursinhalte gibt es Verbesserungspotenzial. Da zahlreiche KundInnen über keinen oder einen negativen Pflichtschulabschluss verfügen, ist ein Hauptschulabschlusskurs angezeigt, bevor ein Eintritt in den Arbeitsmarkt oder eine weiterführende Qualifizierung möglich ist. Es wäre daher wichtig, das Angebot an Hauptschulabschlusskursen zu erweitern, um so den Jugendlichen rasch zu ermöglichen, diese Basisqualifikation nachzuholen.

Speziell die Jugendlichen mit Migrationshintergrund benötigen oftmals Deutschkurse. Das Angebot sollte hier deutlich diversifiziert werden, zum einen in Hinblick auf unterschiedliche Sprachniveaus, zum anderen in Hinblick auf unterschiedliche Zielgruppen. Um am Arbeitsmarkt Fuß zu fassen und nicht nur auf Hilfsjobs angewiesen zu sein, müssen die Jugendlichen über solide Kenntnisse der deutschen Sprache verfügen. Oftmals ist dies selbst bei Jugendlichen, die schon lange in Österreich sind und auch hier die Schule besucht haben, v. a. im schriftlichen Bereich nicht der Fall. Daher sollten verstärkt nicht nur Deutschkurse auf Level A1 und A2 angeboten werden, sondern auch auf höherem Niveau, um die Jugendlichen annähernd auf muttersprachliches Niveau zu führen. In Hinblick auf die Gruppengestaltung sollte verstärkt auf die (schulische) Vorbildung der Jugendlichen geachtet werden, da Jugendliche mit (ausländischer) Matura einem ganz anderen Sprachunterricht folgen können als PflichtschulabsolventInnen. Auch sollten Deutschkurse, die nicht nur die sprachlichen Basiskenntnisse vermitteln, sondern auch die Fachsprache in verschiedenen Berufen berücksichtigen, angeboten werden. Möglichst differenzierte Deutschkurse mit spezifischen Fachmodulen erscheinen in diesem Zusammenhang besonders erfolgversprechend.

Das Berufsorientierungscoaching (BOCO) sollte reformiert und eine qualitativ hochwertige Berufsorientierungsphase angeboten werden, um den Jugendlichen, eine adäquate, individuelle Orientierung zu ermöglichen. Es ist wichtig, den Jugendlichen durch hochwertige Einzel- und Gruppenangebote eine berufliche Perspektive zu geben, damit sie ihren Weg in den Arbeitsmarkt finden können. Die Jugendliche benötigen in diesem Zusammenhang oftmals eher individuelle Unterstützung beim Erstellen von Bewerbungsunterlagen und eine praktische Berufsorientierung denn theoretische Einführungen in verschiedene Berufsbilder. Auch die terminliche Gestaltung der Berufsorientierungsangebote, die $\mathrm{u}$. a. die KundInnen auf eine Lehre in Ausbildungseinrichtungen vorbereiten und somit auch den Berufsschulbeginn mitberücksichtigen müssen, ist zu optimieren. So wurde der Beginn des Berufsorientierungscoachings z. B. mit Anfang August festgesetzt, um einen regulären Berufsschulbesuch im September zu ermöglichen. Jedoch erscheint dazu nur die Hälfte der dafür vorgemerkten KundInnen, da sich viele zu diesem Zeitpunkt im Ausland befinden. Für die Jugendlichen, die sich im September wieder beim AMS melden, bieten sich dann nur mehr wenige Möglichkeiten, da die Orientierungs- und Vorbereitungsphase für eine Lehre in Ausbildungseinrichtungen bereits abgeschlossen ist. Hier müssten Sensibilisierungsmaßnahmen mit den Eltern die Wichtigkeit dieser Termine vermitteln (siehe unten).

Das Angebot an Ausbildungslehrgängen stellt für Jugendliche, die keine betriebliche Lehrstelle finden, eine gute Möglichkeit dar, die Lehrausbildung trotzdem (im Rahmen einer Trägereinrichtung) zu absolvieren. Problematisch ist jedoch, dass sich die Vermittlung jener Jugendlichen, die bis zu ihrer Lehrabschlussprüfung in einer Einrichtung verbleiben, schwieriger gestaltet als bei Jugendlichen, die eine betriebliche Lehre abgeschlossen haben. Um die anschließenden Vermittlungschancen anzugleichen, wäre es erforderlich, die Ausbildung in den Einrichtungen stärker an die betrieblichen An- 
forderungen anzupassen. Möglicherweise wäre es auch hilfreich, im Anschluss an eine Lehrausbildung bei Trägereinrichtungen noch eine Nachbetreuungsphase mit gezielter Unterstützung beim Bewerbungsprozess anzubieten.

Das Angebot von gezielten Qualifizierungen für bestimmte Berufe wird von Kundenseite sehr geschätzt. Um vor allem auch KundInnen, die für eine reguläre Lehrstelle schon zu alt sind, ein vielfältiges Ausbildungsspektrum zu bieten, sollten Qualifizierungsmaßnahmen in verschiedensten Bereichen angeboten werden. Eine Möglichkeit wäre, die Facharbeiterintensivausbildung, die sich vorwiegend auf technische Berufe konzentriert, auch in anderen Berufsfeldern, z. B. im kaufmännischen Bereich oder im Einzelhandel, zu intensivieren, zumal diese laut AMS-BeraterInnen zu einem hohen Vermittlungserfolg führen. Denn gerade für Jugendliche, die noch Zeit zur Entwicklung und Nachreifung brauchen und sich daher erst in einem Alter von 18 oder 19 Jahren für eine Lehrausbildung entscheiden, ist es wichtig, neben der betrieblichen Lehre weitere Qualifizierungsmöglichkeiten anzubieten, damit sie ihre Berufswünsche trotzdem realisieren können.

Unabhängig vom Kursinhalt ist über Maßnahmen der Qualitätssicherung eine Verbesserung des Kursangebots anzustreben. Nicht nur quantitative Zielkontrollen, sondern auch qualitative Untersuchungen und regelmäßige Evaluierungen der Trägereinrichtungen und TrainerInnen sind gefragt, selbst wenn die „offiziellen“ Kursziele erreicht werden. Die Qualität eines Kurses ist nämlich meist vom Engagement des/r einzelnen TrainerIn abhängig. Wenn die AMS-BeraterInnen Kurse besuchen, läuft alles bestens, die KundInnen berichten dann jedoch, dass sie im Internet gesurft haben und der/die Trainerln nur selten anwesend war. Es könnten daher unangekündigte stichprobenartige Besuche der Trägereinrichtungen erfolgen, um die alltäglichen Kursaktivitäten zu beobachten und sich einen Eindruck von der Qualität zu verschaffen. Auch KundInnenBefragungen sind ein hilfreiches Instrument, um zu erheben, wie die Maßnahmen bei der Zielgruppe ankommen.

\section{Organisation}

Das AMS hat durch seine vielfältigen Aufgaben - Verwaltung der „Arbeitslosigkeit“, Vermittlung, Beratung etc. - oftmals widersprüchliche Anforderungen, die sich auch oft in einem teils widersprüchlichen Bild des AMS bei den KundInnen und in der Öffentlichkeit widerspiegeln. $V$. a. die Jugendlichen scheinen oftmals mit den effizienten Verwaltungsabläufen und zuerst gebotenen Informationen überfordert. Erst nach einen „Leidensweg" bzw. wenn eine offenkundige Vermittlungsbarriere vorhanden ist, erfolgt eine intensivere Beratung. Die geschaffene SchulaustreterInnen-Gruppe (SAG), die Personen, die aus der Schule kommen, berät, ist in diesem Sinne sicherlich eine wichtige Maßnahme, um den Erstkontakt mit dem AMS friktionsfrei und ohne Frustration zu gestalten. Hier ist besonders bei der Informationsweitergabe verstärkt auf die Fähigkeiten der einzelnen KundInnen abzuzielen.

In diesem Sinne sollte auch die Homepage des AMS kundenfreundlicher gestaltet werden, um die Informationssuche für die KundInnen zu vereinfachen. Dies gewinnt umso mehr an Relevanz, als dass der Kundenkontakt via Internet z. B. über das e-AMSKonto immer mehr forciert wird. Den KundInnen wird die Möglichkeit geboten, sich via Internet beim AMS an- und abzumelden, Termine zu buchen, Einblick in die Betreuungsvereinbarung zu nehmen etc. Da v. a. Jugendliche den PC zunehmend als primäres Kommunikationsmittel ansehen, ist hier auch auf eine zielgruppengerechte Ausrichtung zu achten. 
In Hinblick auf die Verwaltung der Daten und die Behandlung von Qualifikationen, sind die Vorgaben des AMS für die MitarbeiterInnen nicht immer eindeutig. Dies betrifft zum Beispiel die Anerkennung eines Pflichtschulabschlusses, der im Ausland absolviert wurde. Grundsätzlich wird ein Bildungsabschluss im Ausland als "ohne Pflichtschulabschluss" erfasst, jedoch werden bei gewissen Fällen oder Ländern (z. B. Deutschland) auch Ausnahmen gemacht. Da für die Beraterlnnen der Umgang mit einem Pflichtschulabschluss im Ausland nicht klar ist, sind einheitliche Regelungen (u. a. für Ausnahmefälle) gefragt. Auch sollte insgesamt die Anerkennung von ausländischen Abschlüssen vereinheitlicht werden und am AMS eine entsprechende Anlaufstelle eingerichtet werden bzw. die Kooperation mit Organisationen mit entsprechendem Know-how forciert werden.

Innerhalb des AMS gilt es den Informationsfluss zu optimieren und die MitarbeiterInnen (aus den verschiedensten Zonen und Arbeitsbereichen) über Neuerungen z. B. im Bereich der Kursmaßnahmen standardisiert zu informieren. Auch der Austausch zwischen den verschiedenen Abteilungen sollte stärker forciert werden.

Um die Beratungsleistungen zu optimieren, gilt es auch verstärkt unterstützende Maßnahmen für die AMS-BeraterInnen zu setzen. Das könnte einerseits Supervision sein, um schwierige Fälle aufzuarbeiten, andererseits Maßnahmen, die die Motivation und Teambildung der BeraterInnen fördern. Teambesprechungen und abteilungsübergreifende Austauschforen sollten forciert werden, damit auch alle BeraterInnen einen Gesamtüberblick über die Arbeit im AMS und ihre Klientel erhalten. So könnten auch BeraterInnen aus der Beratungszone, die mit „schwieriger" Klientel zu tun haben, an Vermittlungserfolgen partizipieren. Auch könnte ein verstärktes Rückmeldesystem bei erfolgreicher Vermittlung durch den/die Jugendliche angedacht werden, sodass den BeraterInnen auch die positiven Resultate ihrer Arbeit sichtbar werden.

Um das Image des AMS als Dienstleistungsunternehmen am Arbeitsmarkt in der Bevölkerung im Allgemeinen und als Erstanlaufstelle für Jugendliche im Besonderen zu verbessern, gilt es eine breitere Öffentlichkeitsarbeit zu gestalten. Dabei spielen Kooperationen mit anderen relevanten Akteuren (siehe unten), eine wesentliche Rolle.

\section{Diversity-Aktivitäten}

Um das Thema Diversity Management noch stärker im Rahmen des AMS zu implementieren, sollten Vorträge und Lehrgänge (z. B. SIQUA-Lehrgang) weiter ausgebaut werden. Dabei wäre es wichtig nicht nur die interessierten MitarbeiterInnen für das Thema zu begeistern, sondern auch jene beizuziehen, die sich damit noch weniger identifizieren können. Eine andere Möglichkeit wäre, die interne Weitergabe von Diversity Know-how stärker zu forcieren und einen internen Informationsaustausch anzuregen. So könnten MitarbeiterInnen, die Seminare oder Lehrgänge besucht haben, die Informationen an ihre KollegInnen weitergeben.

Des Weiteren gilt es, neben der weiteren Rekrutierung von BeraterInnen mit Migrationshintergrund deren Ressourcen stärker zu nutzen. Dies betrifft jedoch nicht nur die sprachlichen Fähigkeiten, sondern auch die persönlichen Erfahrungen der Beraterlnnen in Zusammenhang mit dem Thema Migration. Es wäre wichtig, dass die BeraterInnen mit Migrationshintergrund innerhalb des AMS von ihren eigenen „Migrationserlebnissen" berichten, damit auch die übrigen MitarbeiterInnen vom Erfahrungsschatz dieser BeraterInnen profitieren können. Die Erfahrungen und Standpunkte von Personen mit Migrationshintergrund können den BeraterInnen aber auch durch Vorträge von externen Personen nähergebracht werden. Der Einblick in die Situation und Le- 
benswelt von MigrantInnen ist eine hilfreiche Hintergrundinformation für die Beratungsgespräche mit dieser Kundengruppe. Kooperationen mit MigrantInnenorganisationen sind dabei von besonderer Bedeutung (siehe unten).

\section{Kooperation mit relevanten Akteuren}

Das AMS Jugendliche übernimmt ihre KundInnen von der Schule und vermittelt sie auf dem Arbeitsmarkt in Unternehmen. Damit muss sie die Jugendlichen mit den Fähigkeiten und Kenntnissen, die sie sich im Rahmen des Schulsystems aneignen konnten, in Unternehmen platzieren, die Anforderungen und Erwartungen an diese stellen, die wirtschaftlichen Notwendigkeiten gehorchen. Damit tritt das AMS als Vermittler zwischen zwei Akteurswelten mit unterschiedlichen Logiken auf und eine intensive Zusammenarbeit mit diesen ist unabdingbar, um den Aufgaben, die an das AMS gestellt werden, zu erfüllen.

Viele schulische Probleme werden zurzeit dem AMS übertragen. Zahlreiche Jugendliche absolvieren die 4. Hauptschulklasse nicht mehr, wenn sie eine Klasse wiederholen mussten, schließen ihre Schullaufbahn mit negativem Pflichtschulabschluss ab oder werden durch die Pflichtschule „getragen“. Diese Jugendlichen weisen oft erhebliche Leistungsdefizite auf und sind noch nicht reif für den Arbeitsmarkt. Dies ist $u$. a. durch das sinkende Leistungsniveau in den Schulen mitbedingt. Ein weiteres Problem ist, dass die schulischen Standards nicht mit dem Anforderungen des AMS und des Arbeitsmarktes überein stimmen. Den Jugendlichen wird beispielsweise empfohlen, bereits nach der 2. oder 3. Hauptschulklasse in eine polytechnische Schule zu wechseln, obwohl innen dann der Hauptschulabschluss fehlt, der anschließend nachgeholt werden muss. Auch die Schulabschlüsse und Noten der KundInnen werden als wenig aussagekräftig und kaum vergleichbar erlebt. Die Benotung sagt oftmals nichts über die tatsächlichen Fähigkeiten der KundInnen aus. Selbst positive Abschlüsse können mit erheblichen Leistungsdefiziten verbunden sein, die eine Vermittlung am Arbeitsmarkt erschweren. Das AMS muss daher den Jugendlichen häufig noch grundlegende Qualifikationen vermitteln, obwohl dies eigentlich die Aufgabe der Schule wäre.

Um die verschiedenen Standards von Schulen und AMS anzugleichen und den Umgang mit leistungsschwachen, schwierigen Jugendlichen zu verbessern, sollte das AMS die Zusammenarbeit mit den schulischen Akteuren intensivieren. Nur wenn gemeinsam an Lösungskonzepten gearbeitet wird, kann den Jugendlichen geholfen werden, damit ein erfolgreicher Eintritt in den Arbeitsmarkt gelingt. So könnten die Schulen das AMS entlasten, indem auf den Erfordernissen des Arbeitsmarkts abgestimmte Bewerbungsunterlagen erstellt werden oder schon in der Schulzeit Berufsorientierungsmaßnahmen gesetzt werden. Da die Jugendlichen vor allem mit praktischen Erprobungen und aktivem Hineinschnuppern in verschiedene Berufsbereiche zu begeistern sind, sollten diese Berufsorientierungsaktivitäten - obwohl sie im schulischen Rahmen stattfinden - auch praktische Aktivitäten beinhalten, um die vielfältigen Aufgabenbereiche in verschiedenen Berufen möglichst anschaulich zu vermitteln.

Darüber hinaus gilt es auch die Präsenz des AMS in den Schulen zu steigern, um die Jugendlichen bereits während ihrer Schullaufbahn auf das AMS und dessen Berufsberatungsangebote (z. B. im Rahmen der BerufsInfoZentren (BIZ)) aufmerksam zu machen. Besonders Jugendliche mit Migrationshintergrund, deren Eltern wenig Information zum österreichischen Bildungssystem und zum Arbeitsmarkt haben, könnten von einem verstärkten Informationsfluss in der Schule profitieren. Dadurch kann vermieden werden, dass Jugendliche sich spät an das AMS wenden und wertvolle Zeit zur Lehrstellen- oder Arbeitsplatzsuche verstreicht. 
In diesem Zusammenhang sei auch auf die verstärkte Einbindung der Community bzw. von MigrantInnenorganisationen verwiesen. Über diese Organisationen können wichtige Informationen über Gegebenheiten und Notwendigkeiten am österreichischen Arbeitsmarkt verbreitet werden und v. a. auch die Eltern erreicht werden, damit sie ihre Kinder bei einer Qualifizierung und bei der Integration in den Arbeitsmarkt unterstützen.

Um den Vermittlungsprozess zu erleichtern, ist auch eine vermehrte Kooperation mit der Wirtschaft gefragt. Die AMS-BeraterInnen gehen davon aus, dass ein Großteil der Unternehmen dem AMS seine freien Stellen nicht meldet und lediglich ein geringer Teil der Betriebe in Wien dem AMS bekannt gibt, wenn Arbeitskräfte gesucht werden. Es wäre daher wichtig, im Rahmen von Marketingaktivitäten Unternehmen zu motivieren, ihre freien Stellen zu melden und die Suche nach Arbeitnehmerlnnen verstärkt über das AMS abwickeln zu lassen. Andererseits bleiben offene Stellen als solche lange gekennzeichnet, obwohl bereits ein/e geeignete/r Kandidatln gefunden wurde. Damit bewerben sich Jugendliche auf Stellen, die bereits besetzt sind, was die Kompetenz des AMS als Stellenvermittler schmälert. Daher wäre in diesem Bereich eine größere Disziplin der Unternehmen einzufordern und kurzfristiger die freien Stellen zu aktualisieren.

Ein weiteres Problem in Zusammenarbeit mit den Unternehmen ist, dass sie kaum Rückmeldungen auf Bewerbungen geben. Selbst wenn die Bewerbung des/der Kundln ungeeignet ist, sollte zumindest eine Absage übermittelt werden. Für die KundInnen ist es sehr frustrierend, wenn sie zahlreiche Bewerbungen versenden und keinerlei Rückmeldung erhalten. Hier sollte das AMS verstärkt die Zusammenarbeit mit den Betrieben suchen, um eine Erhöhung der Rückmeldungen zu erhalten.

Zudem müssen die Unternehmen verstärkt für die Migrationsthematik sensibilisiert werden, damit (unbewusste) Diskriminierungsmuster nicht greifen. Beratung und Betreuung für Unternehmen könnte diese motivieren, verstärkt Jugendlichen mit Migrationshintergrund eine Lehrstelle oder einen Arbeitsplatz anzubieten. 